\title{
Some New Covering-Based Multigranulation Fuzzy Rough Sets and Corresponding Application in Multicriteria Decision Making
}

\author{
Zaibin Chang $(\mathbb{D})$ and Lingling Mao ic \\ Department of Basic Education, Xi'an Traffic Engineering University, Xi'an 710300, China \\ Correspondence should be addressed to Lingling Mao; maolinglingm@163.com
}

Received 26 July 2021; Revised 13 October 2021; Accepted 21 October 2021; Published 19 November 2021

Academic Editor: Ching-Feng Wen

Copyright (c) 2021 Zaibin Chang and Lingling Mao. This is an open access article distributed under the Creative Commons Attribution License, which permits unrestricted use, distribution, and reproduction in any medium, provided the original work is properly cited.

\begin{abstract}
Multigranulation rough set theory is an important tool to deal with the problem of multicriteria information system. The notion of fuzzy $\beta$-neighborhood has been used to construct some covering-based multigranulation fuzzy rough set (CMFRS) models through multigranulation fuzzy measure. But the $\beta$-neighborhood has not been used in these models, which can be seen as the bridge of fuzzy covering-based rough sets and covering-based rough sets. In this paper, the new concept of multigranulation fuzzy neighborhood measure and some types of covering-based multigranulation fuzzy rough set (CMFRS) models based on it are proposed. They can be seen as the further combination of fuzzy sets: covering-based rough sets and multigranulation rough sets. Moreover, they are used to solve the problem of multicriteria decision making. Firstly, the definition of multigranulation fuzzy neighborhood measure is given based on the concept of $\beta$-neighborhood. Moreover, four types of CMFRS models are constructed, as well as their characteristics and relationships. Then, novel matrix representations of them are investigated, which can satisfy the need of knowledge discovery from large-scale covering information systems. The matrix representations can be more easily implemented than set representations by computers. Finally, we apply them to manage the problem of multicriteria group decision making (MCGDM) and compare them with other methods.
\end{abstract}

\section{Introduction}

Covering-based rough sets $[1,2]$ enriched classical rough sets $[3,4]$ to deal with the type of covering data. In fact, this data is the collection of subsets of a universe (the set of all objects). Based on it, many covering-based rough set models $[5,6]$ were constructed to manage some practical problems, such as attribute reduction $[7,8]$ and rule synthesis $[9,10]$.

As the same with rough set theory, Zadeh's fuzzy set theory [11] can also deal with the problem of imperfect knowledge. There are many researchers who investigated their connection and distinction $[12,13]$. To extend fuzzy set theory and improve its application, several generalizations of fuzzy sets have been proposed, such as intuitionistic fuzzy sets [14], soft set [15], bipolar-valued fuzzy sets [16, 17], hesitant bipolar-valued fuzzy soft sets [18], a historical account of types of fuzzy sets [19], and single-valued neutrosophic sets [20].
Recently, the collection of subsets of a universe has been generalized to a collection of fuzzy subsets of a universe. That is to say, coverings have been generalized to fuzzy covering under a special condition. Hence, covering-based rough sets have been extended to covering-based fuzzy rough sets under fuzzy coverings, such as fuzzy covering tough sets [21] and generalized fuzzy covering tough sets $[22,23]$. But in the condition of fuzzy covering, there is a constraint " 1 ". Then, Ma [24] proposed the concept of fuzzy $\beta$-covering approximation space ( $\beta$-FcaS) by extending " 1 " to a parameter $\beta$. Motivated by Ma's work, many significative researches have been done. For example, Zhang and Wang [25] studied the $\beta$-FcaS further, D'eer et al. $[26,27]$ investigated relationships among fuzzy neighborhood operators. Yang and $\mathrm{Hu}[28,29]$ constructed other covering-based fuzzy rough set models. Zhang et al. [30] used fuzzy measures and Choquet integrals in fuzzy covering rough sets for solving the decision making. 
However, all these above works have been done under a $\beta$-FcaS. Hence, Zhan et al. [31] extended a fuzzy $\beta$-covering to $n$-fuzzy $\beta$-coverings. They also proposed two important kinds of neighborhoods, which are the fuzzy $\beta$-neighborhood $\widetilde{\mathcal{N}}_{\widehat{\mathrm{C}}(x)}^{\beta}$ and the $\beta$-neighborhood $\overline{\mathcal{N}}_{\widehat{\mathrm{C}}(x)}^{\beta}$ of $x$. Zhan et al. [31] used the concept of fuzzy $\beta$-neighborhood to establish two types of covering-based multigranulation fuzzy rough sets (CMFRSs). Moreover, based on the concept of fuzzy $\beta$-neighborhood, they give the notion of multigranulation fuzzy measure as follows:

$$
\widetilde{D}_{\widehat{\mathbf{C}}_{i}}^{\beta}(x, y)=\frac{\sum_{j=1}^{m}\left(\tilde{\mathcal{N}}_{\widehat{\mathrm{C}}_{i}\left(x_{j}\right)}^{\beta}(x) \bigvee \widetilde{\mathcal{N}}_{\widehat{\mathrm{C}}_{i}\left(x_{j}\right)}^{\beta}(y)\right)}{\sum_{j=1}^{m}\left(\tilde{\mathcal{N}}_{\widehat{\mathrm{C}}_{i}\left(x_{j}\right)}^{\beta}(x) \bigwedge \widetilde{\mathcal{N}}_{\widehat{\mathrm{C}}_{i}\left(x_{j}\right)}^{\beta}(y)\right)} .
$$

Then, it was used to construct another four types of CMFRSs. But it is time-consuming to compute them when a large-scale covering is given. On the other hand, the concept of $\beta$-neighborhood is not used in [31]. Since the concept of $\beta$-neighborhood can be used for any fuzzy $\beta$-covering to induce a covering, it can be seen as the bridge of fuzzy covering-based rough sets and covering-based rough sets. That is to say, some methods in covering-based rough sets can be used in fuzzy covering-based rough sets from the viewpoint of the concept of $\beta$-neighborhood. In this paper, we use the concept of $\beta$-neighborhood to present the notion of multigranulation fuzzy neighborhood measure in this paper. Moreover, we construct some types of CMFRSs by it. follows:

The motivation and content of this paper are shown as

(i) To solve the problem of time-consuming for computing the multigranulation fuzzy measure when a large-scale covering is given, the notion of multigranulation fuzzy neighborhood measure is given by the concept of $\beta$-neighborhood as follows:

$$
D_{\widehat{\mathbf{C}}_{i}}^{\beta}(x, y)=\frac{\left|\overline{\mathcal{N}}_{\widehat{\mathbf{C}}_{i}(x)}^{\beta} \bigcap \overline{\mathcal{N}}_{\widehat{\mathbf{C}}_{i}(y)}^{\beta}\right|}{\left|\overline{\mathcal{N}}_{\widehat{\mathbf{C}}_{i}(x)}^{\beta} \bigcup \overline{\mathcal{N}}_{\widehat{\mathbf{C}}_{i}(y)}^{\beta}\right|} .
$$

It can be seen as the complement of the existing work and the first motivation of this paper. Based on this new notion, four types of CMFRS models are constructed. They are covering-based optimistic (or pessimistic) multigranulation neighborhood fuzzy rough set models and $\alpha$-covering-based optimistic (or pessimistic) multigranulation neighborhood fuzzy rough set models, where $\alpha \in[0,1]$.

(ii) Then, we investigate them from the following three ways: characteristics, relationships, and matrix representations. Moreover, we define the matrix representation of all multigranulation fuzzy neighborhood measures, which is symmetric, to study the problem of their matrix representations. The matrix methods can satisfy the need of knowledge discovery. It can well solve the time-consuming problem when a large-scale covering is given, which is the second motivation of this paper.

(iii) Finally, as an application, we use them to deal with the problem of multicriteria decision making (MCGDM) and compare with other methods.

The rest of this paper is organized as follows: Section 2 shows some definitions about fuzzy covering-based rough sets. In Section 3, we give covering-based (and $\alpha$-coveringbased) optimistic multigranulation neighborhood fuzzy rough set models and their characteristics in Section 3.1 and covering-based (and $\alpha$-covering-based) pessimistic multigranulation neighborhood fuzzy rough set models and their characteristics in Section 3.2. Section 3.3 gives their relationships. In Section 4, we use matrix approaches to compute these CMFRS models. Section 5 gives an application to show that these CMFRS models can be used to solve the problem of MCGDM. Section 6 is the conclusions and further work.

\section{Basic Definitions}

This section shows several fundamental definitions related to fuzzy covering-based rough sets. It is supposed that $U$ is a nonempty and finite set called universe.

To combine covering-based rough sets and fuzzy sets, $\mathrm{Ma}$ [24] presented the notion of $\beta$-FcaS.

Definition 1 (see [24]). Let $F(U)$ be the fuzzy power set of $U$. For each $\beta \in(0,1]$, we call $\widehat{C}=\left\{C_{1}, C_{2}, \ldots, C_{m}\right\}$, with $C_{i} \in F(U)(i=1,2, \ldots, m)$, a fuzzy $\beta$-covering of $U$, if $\left(\cup_{i=1}^{m} C_{i}\right)(x) \geq \beta$ for any $x \in U$. We also call $(U, \widehat{C})$ a $\beta$-FcaS.

In a $\beta$-FcaS, there are two types of neighborhoods, which are shown in Definition 2.

Definition 2 (see [24]). Let $(U, \widehat{\mathbf{C}})$ be a $\beta$-FcaS. For any $x \in U$, the fuzzy $\beta$-neighborhood $\widetilde{\mathcal{N}}_{\widehat{\mathrm{C}}(x)}^{\beta}$ and the $\beta$-neighborhood $\overline{\mathcal{N}}_{\widehat{\mathrm{C}}(x)}^{\beta}$ of $x$ are defined as follows:

$$
\begin{aligned}
& \widetilde{\mathcal{N}}_{\widehat{\mathbf{C}}(x)}^{\beta}=\bigcap\{C \in \widehat{\mathbf{C}}: C(x) \geq \beta\}, \\
& \overline{\mathcal{N}}_{\widehat{\mathbf{C}}(x)}^{\beta}=\left\{y \in U: \widetilde{\mathcal{N}}_{\widehat{\mathbf{C}}(x)}^{\beta}(y) \geq \beta\right\} .
\end{aligned}
$$

Then, Zhan et al. [31] generalized a fuzzy $\beta$-covering to $n$-fuzzy $\beta$-coverings. We call $\widetilde{\Gamma}=\left\{\widehat{\mathbf{C}}_{1}, \ldots, \widehat{\mathbf{C}}_{n}\right\}$ a $n$-fuzzy $\beta$-coverings of $U$, where $\widehat{\mathbf{C}}_{i}$ is a fuzzy $\beta$-covering of $U$ for any $i \in\{1,2, \ldots, n\}$. Then, two types of covering-based multigranulation rough sets are proposed.

Definition 3 (see [31]). Let $\widetilde{\Gamma}=\left\{\widehat{\mathbf{C}}_{1}, \ldots, \widehat{\mathbf{C}}_{n}\right\}$ be an $n$-fuzzy $\beta$-coverings of $U$. For any $A \in F(U)$, the OMGFRLAO $\underline{R}_{\sum_{i=1}^{n} \widehat{\mathbf{c}}_{i}}(A)$ and the OMGFRUAO $\bar{R}_{\sum_{i=1}^{n} \widehat{\mathbf{c}}_{i}}(A)$ are defined as follows: for any $x \in U$, 


$$
\begin{aligned}
& \underline{R}_{\sum_{i=1}^{n} \widehat{\mathrm{C}}_{i}}^{O}(A)(x)=\bigvee_{i=1}^{n} \bigwedge_{y \in U}\left\{\left(1-\widetilde{\mathcal{N}}_{\widehat{\mathrm{C}}_{i}(x)}^{\beta}(y)\right) \bigvee A(y)\right\}, \\
& \bar{R}_{\sum_{i=1}^{n} \widehat{\mathrm{C}}_{i}}(A)(x)=\bigvee_{i=1}^{n} \bigvee_{y \in U}\left\{\widetilde{\mathcal{N}}_{\widehat{\mathrm{C}}_{i}(x)}^{\beta}(y) \bigwedge A(y)\right\} .
\end{aligned}
$$

The PMGFRLAO $\underline{R}_{\sum_{i=1}^{n} \widehat{\mathrm{C}}_{i}}(A)$ and the PMGFRUAO $\bar{R}_{\sum_{i=1}^{n} \widehat{\mathrm{C}}_{i}}(A)$ are defined as follows: for any $x \in U$,

$$
\begin{aligned}
& \underline{R}_{\sum_{i=1}^{n} \widehat{\mathrm{C}}_{i}}^{P}(A)(x)=\bigwedge_{i=1}^{n} \bigwedge_{y \in U}\left[\left(1-\widetilde{\mathcal{N}}_{\widehat{\mathrm{C}}_{i}(x)}^{\beta}(y)\right) \bigvee A(y)\right], \\
& \bar{R}_{\sum_{i=1}^{n} \widehat{\mathrm{C}}_{i}}(A)(x)=\bigvee_{i=1}^{n} \bigvee_{y \in U}\left[\tilde{\mathcal{N}}_{\widehat{\mathrm{C}}_{i}(x)}^{\beta}(y) \bigwedge A(y)\right] .
\end{aligned}
$$

\section{Some Types of CMFRSs}

In this section, we propose the notion of multigranulation fuzzy neighborhood measure under $\beta$-neighborhoods. Then, four types of CMFRSs are constructed through the notion of multigranulation fuzzy neighborhood. Finally, some relationships among them are investigated.

3.1. Covering-Based Optimistic Multigranulation Fuzzy Rough Sets. Two types of covering-based optimistic multigranulation fuzzy rough sets are proposed in this section. Firstly, the notion of multigranulation fuzzy neighborhood measure is presented in Definition 4.

Definition 4. Let $\widetilde{\Gamma}=\left\{\widehat{\mathbf{C}}_{1}, \ldots, \widehat{\mathbf{C}}_{n}\right\}$ be a $n$-fuzzy $\beta$-coverings of $U$. For any $i \in\{1,2, \ldots, n\}$ and $x, y \in U$, we call

$$
D_{\widehat{\mathbf{C}}_{i}}^{\beta}(x, y)=\frac{\left|\overline{\mathcal{N}}_{\widehat{\mathbf{C}}_{i}(x)}^{\beta} \bigcap \overline{\mathcal{N}}_{\widehat{\mathbf{C}}_{i}(y)}^{\beta}\right|}{\left|\overline{\mathcal{N}}_{\widehat{\mathbf{C}}_{i}(x)}^{\beta} \bigcup \int \overline{\mathcal{N}}_{\widehat{\mathbf{C}}_{i}(y)}^{\beta}\right|}
$$

a multigranulation fuzzy neighborhood measure (MFNM) of $x$ and $y$ under $\widehat{\mathbf{C}}_{i}$.

By Definition 4 , for any $i \in\{1,2, \ldots, n\}$ and $x, y \in U$, we have $D_{\widehat{\mathbf{C}}_{i}}^{\beta}(x, x)=1, D_{\widehat{\mathbf{C}}_{i}}^{\beta}(x, y)=D_{\widehat{C}_{i}}^{\beta}(y, x)$ and $0 \leq D_{\widehat{C}_{i}}^{\beta}(x$, $y) \leq 1$.

Example 1. $\Gamma=\left\{\widehat{\mathbf{C}}_{1}, \widehat{\mathbf{C}}_{2}\right\}$ is a family of fuzzy $\beta$-coverings of $U$, where $\beta=0.6$ and $\widehat{\mathbf{C}}_{i}=\left\{C_{i 1}, C_{i 2}, C_{i 3}, C_{i 4}\right\}(i=1,2)$. They are shown in Tables 1 and 2, respectively.

Then, $\widetilde{\mathcal{N}}_{\widehat{\mathrm{C}}_{1}\left(x_{j}\right)}^{0.6}$ and $\widetilde{\mathcal{N}}_{\widehat{\mathrm{C}}_{2}\left(x_{j}\right)}^{0.6}$ are shown in Tables 3 and 4 , respectively.

Hence, $\overline{\mathcal{N}}_{\widehat{\mathrm{C}}_{1}\left(x_{j}\right)}^{0.6}$ and $\overline{\mathcal{N}}_{\hat{\mathrm{C}}_{2}\left(x_{j}\right)}^{0.6}$ are shown in Tables 5 and 6 , respectively.

Therefore, $D_{\widehat{C}_{1}}^{0.6}\left(x_{j}, x_{k}\right)$ and $D_{\widehat{C}_{2}}^{0.6}\left(x_{j}, x_{k}\right)$ are shown in Tables 7 and 8 , respectively.

Then, the first type of CMFRSs is presented in Definition 5.
Definition 5. Let $\widetilde{\Gamma}=\left\{\widehat{\mathbf{C}}_{1}, \ldots, \widehat{\mathbf{C}}_{n}\right\}$ be a $n$-fuzzy $\beta$-coverings of $U$. For any $A \in F(U)$, the optimistic multigranulation neighborhood fuzzy lower approximation operator (OMNFLAO) $S \underline{R}_{\sum_{i=1}^{n} \widehat{\mathrm{C}}_{i}}^{O}(A)$ and the optimistic multigranulation neighborhood fuzzy upper approximation operator (OMNFUAO) $S \bar{R}_{\sum_{i=1}^{n} \widehat{C}_{i}}(A)$ are defined as follows: for any $x \in U$,

$$
\begin{aligned}
& S \underline{R}_{\sum_{i=1}^{n} \widehat{\mathrm{C}}_{i}}^{O}(A)(x)=\bigvee_{i=1}^{n} \bigwedge_{y \in U}\left\{\left(1-D_{\widehat{\mathrm{C}}_{i}}^{\beta}(x, y)\right) \bigvee A(y)\right\}, \\
& S \bar{R}_{\sum_{i=1}^{n} \widehat{\mathrm{C}}_{i}}^{O}(A)(x)=\bigwedge_{i=1}^{n} \bigvee_{y \in U}\left\{D_{\widehat{\mathrm{C}}_{i}}^{\beta}(x, y) \bigwedge A(y)\right\} .
\end{aligned}
$$

In Definition 5, we call $A$ a covering-based optimistic multigranulation neighborhood fuzzy rough set; otherwise, we call it a covering-based optimistic multigranulation neighborhood definable set.

Example 2 (continued from Example 1). Let $A=(0.8 /$ $\left.x_{1}\right)+\left(0.4 / x_{1}\right)+\left(0.6 / x_{1}\right)+\left(0.2 / x_{1}\right)+\left(0.5 / x_{5}\right)$ Then, $S \underline{R}_{\sum_{i=1}^{O} \hat{\mathrm{C}}_{i}}^{O}(A)=\left(0.6667 / x_{1}\right)+\left(0.4 / x_{2}\right)+\left(0.6 / x_{3}\right)+\left(0.2 / x_{4}\right)+$ $\left(0.5 / x_{5}\right)$ and $S \bar{R}_{\sum_{i=1}^{2} \widehat{\mathrm{C}}_{i}}(A)=\left(0.8 / x_{1}\right)+\left(0.4 / x_{2}\right)+(0.6 /$ $\left.x_{3}\right)+\left(0.333 / x_{4}\right)+\left(0.5 / x_{5}\right)$.

Some characteristics of the OMNFLAO $S \underline{R}_{\sum_{i=1}^{n}}^{O} \widehat{\mathrm{C}}_{i}(A)$ and the OMNFUAO $S \bar{R}_{\sum_{i=1}^{n} \widehat{\mathrm{C}}_{i}}(A)$ are presented as follows.

Proposition 1. Let $\widetilde{\Gamma}=\left\{\widehat{\mathbf{C}}_{1}, \ldots, \widehat{\mathbf{C}}_{n}\right\}$ be an $n$-fuzzy $\beta$-coverings of $U$. Then, for any $A, B \in F(U)$, the following statements hold:

(1LH) $S \underline{R}_{\sum_{i=1}^{n}}^{O} \widehat{\mathrm{C}}_{i}(A) \subseteq A \subseteq S \bar{R}_{\sum_{i=1}^{n} \widehat{\mathrm{C}}_{i}}(A)$

(2L) If $A \subseteq B$, then $S \underline{R}_{\sum_{i=1}^{O} \widehat{\mathrm{C}}_{i}}(A) \subseteq S \underline{R}_{\sum_{i=1}^{n}}^{O} \widehat{\mathrm{C}}_{i}(B)$

(2H) If $A \subseteq B$, then $S \bar{R}_{\sum_{i=1}^{n} \widehat{\mathrm{C}}_{i}}^{O}(A) \subseteq S \bar{R}_{\sum_{i=1}^{n} \widehat{\mathrm{C}}_{i}}(B)$

(3L) $\quad S \underline{R}_{\sum_{i=1}^{n} \widehat{\mathrm{C}}_{i}}^{O}(A \cap B)=S \underline{R}_{\sum_{i=1}^{n} \widehat{\mathrm{C}}_{i}}^{O}(A) \cap S \underline{R}_{\sum_{i=1}^{n} \widehat{\mathrm{C}}_{i}}^{O}(B)$,

$\underline{S R}_{\sum_{i=1}^{n} \widehat{\mathbf{C}}_{i}}(A \cup B) \supseteq S \underline{R}_{\sum_{i=1}^{O} \widehat{\mathbf{C}}_{i}}(A) \cup S \underline{R}_{\sum_{i=1}^{O} \widehat{\mathbf{c}}_{i}}(B)$

(3H) $\quad S \bar{R}_{\sum_{i=1}^{n} \widehat{\mathrm{C}}_{i}}^{O}(A \cap B) \subseteq S \bar{R}_{\sum_{i=1}^{n} \widehat{\mathrm{C}}_{i}}(A) \cap S \bar{R}_{\sum_{i=1}^{n} \widehat{\mathrm{C}}_{i}}(B)$,

$S \bar{R}_{\sum_{i=1}^{n} \widehat{\mathrm{C}}_{i}}(A \cup B)=S \bar{R}_{\sum_{i=1}^{n} \widehat{\mathrm{c}}_{i}}(A) \cup S \bar{R}_{\sum_{i=1}^{n} \widehat{\mathrm{c}}_{i}}(B)$

(4LH) $\quad\left(S \underline{R}_{\sum_{i=1}^{n} \widehat{\mathrm{c}}_{i}}^{O}(A)\right)^{\prime}=S \bar{R}_{\sum_{i=1}^{n} \widehat{\mathrm{C}}_{i}}\left(A^{\prime}\right), \quad\left(S \bar{R}_{\sum_{i=1}^{n} \widehat{\mathrm{c}}_{i}}(A)\right)^{\prime}$

$=S \underline{R}_{\sum_{i=1}^{n}}^{n_{\mathbf{C}_{i}}}\left(A^{\prime}\right)$

Proof

(1LH). For any $x \in U$ and $i=1,2, \ldots, n, D_{\widehat{C}}^{\beta}(x, x)=1$ by Definition 4 . Then, $A(x)=A(x) \wedge D_{\widehat{\mathrm{C}}_{i}}^{\beta}(x, x) \leq \mathrm{V}_{y \in U}$ $\left\{D_{\widehat{\mathrm{C}}_{i}}^{\beta}(x, y) \wedge A(y)\right\}$. Hence, $A(x) \leq \wedge_{i=1}^{n} \vee_{y \in U}\{D$ 
Table 1: $\widehat{\mathbf{C}}_{1}$.

\begin{tabular}{lllll}
\hline$U$ & $C_{11}$ & $C_{12}$ & $C_{13}$ & \\
\hline$x_{1}$ & 0.8 & 0.4 & 0.3 & \\
$x_{2}$ & 0.3 & 0.6 & 0.5 & \\
$x_{3}$ & 0.7 & 0.2 & 0.6 & \\
$x_{4}$ & 0.8 & 0.7 & 0.7 & 0.4 \\
$x_{5}$ & 0.8 & 0.9 & 0.4 & 0.8 \\
\hline
\end{tabular}

TABle 2: $\widehat{\mathbf{C}}_{2}$.

\begin{tabular}{lllll}
\hline$U$ & $C_{21}$ & $C_{22}$ & $C_{23}$ & \\
\hline$x_{1}$ & 0.4 & 0.7 & 0.7 & \\
$x_{2}$ & 0.3 & 0.8 & 0.6 & \\
$x_{3}$ & 0.8 & 0.6 & 0.7 & 0.3 \\
$x_{4}$ & 0.6 & 0.3 & 0.3 & 0.4 \\
$x_{5}$ & 0.7 & 0.4 & 0.6 & 0.7 \\
\hline
\end{tabular}

TABLe 3: $\widetilde{\mathcal{N}}_{\mathrm{C}_{1}\left(x_{j}\right)}^{0.6}(j=1,2, \ldots, 5)$.

\begin{tabular}{|c|c|c|c|c|c|}
\hline$U$ & $x_{1}$ & $x_{2}$ & $x_{3}$ & $x_{4}$ & $x_{5}$ \\
\hline$\tilde{\mathcal{N}}_{\widehat{\mathrm{C}}_{1}\left(x_{1}\right)}^{0.6}$ & 0.7 & 0.3 & 0.7 & 0.5 & 0.6 \\
\hline$\widetilde{\mathcal{N}}_{\mathrm{C}_{1}\left(x_{2}\right)}^{0.6}$ & 0.4 & 0.6 & 0.2 & 0.7 & 0.9 \\
\hline$\widetilde{\mathcal{N}}_{\widehat{\mathrm{C}}_{1}\left(x_{3}\right)}^{0.6}$ & 0.3 & 0.3 & 0.6 & 0.5 & 0.4 \\
\hline$\widetilde{\mathcal{N}}_{\widehat{\mathrm{C}}_{1}\left(x_{4}\right)}^{0.6}$ & 0.3 & 0.3 & 0.2 & 0.7 & 0.4 \\
\hline$\widetilde{\mathcal{N}}_{\widehat{\mathrm{C}}_{1}\left(x_{5}\right)}^{0.6}$ & 0.4 & 0.3 & 0.2 & 0.5 & 0.6 \\
\hline
\end{tabular}

TABLe 4: $\widetilde{\mathcal{N}}_{\mathrm{C}_{2}\left(x_{j}\right)}^{0.6}(j=1,2, \ldots, 5)$.

\begin{tabular}{|c|c|c|c|c|c|}
\hline$U$ & $x_{1}$ & $x_{2}$ & $x_{3}$ & $x_{4}$ & $x_{5}$ \\
\hline$\widetilde{\mathcal{N}}_{\widehat{\mathrm{C}}_{2}\left(x_{1}\right)}^{0.6}$ & 0.7 & 0.6 & 0.6 & 0.3 & 0.4 \\
\hline$\tilde{\mathcal{N}}_{\widehat{\mathbf{C}}_{2}\left(x_{2}\right)}^{0.6}$ & 0.7 & 0.6 & 0.6 & 0.3 & 0.4 \\
\hline$\tilde{\mathcal{N}}_{\widehat{\mathbf{C}}_{2}\left(x_{3}\right)}^{0.6}$ & 0.4 & 0.3 & 0.6 & 0.3 & 0.4 \\
\hline$\tilde{\mathcal{N}}_{\widehat{\mathbf{C}}_{2}\left(x_{4}\right)}^{0.6}$ & 0.3 & 0.3 & 0.4 & 0.6 & 0.7 \\
\hline$\widetilde{\mathcal{N}}_{\hat{\mathrm{C}}_{2}\left(x_{5}\right)}^{0.6}$ & 0.3 & 0.3 & 0.4 & 0.3 & 0.6 \\
\hline
\end{tabular}

TABle 5: $\overline{\mathcal{N}}_{\mathrm{C}_{1}\left(x_{j}\right)}^{0.6}(j=1,2, \ldots, 5)$.

\begin{tabular}{|c|c|c|c|c|}
\hline$\overline{\mathcal{N}}_{\widehat{\mathrm{C}}_{1}\left(x_{1}\right)}^{0.6}$ & $\overline{\mathcal{N}}{\overline{\mathrm{C}_{1}}}_{\left(x_{2}\right)}^{0.6}$ & $\overline{\mathcal{N}}_{\hat{\mathrm{C}}_{1}\left(x_{3}\right)}^{0.6}$ & $\overline{\mathcal{N}}_{\mathrm{C}_{1}\left(x_{4}\right)}^{0.6}$ & $\overline{\mathcal{N}}_{\mathrm{C}_{1}\left(x_{5}\right)}^{0.6}$ \\
\hline$\left\{x_{1}, x_{3}, x_{5}\right\}$ & $\left\{x_{2}, x_{4}, x_{5}\right\}$ & $\left\{x_{3}\right\}$ & $\left\{x_{4}\right\}$ & $\left\{x_{5}\right\}$ \\
\hline
\end{tabular}

TABle 6: $\overline{\mathcal{N}}_{\tilde{\mathrm{C}}_{2}\left(x_{j}\right)}^{0.6}(j=1,2, \ldots, 5)$.

\begin{tabular}{|c|c|c|c|c|}
\hline$\overline{\mathcal{N}}_{\hat{\mathrm{C}}_{2}\left(x_{1}\right)}^{0.6}$ & $\overline{\mathcal{N}}_{\mathrm{C}_{2}\left(x_{2}\right)}^{0.6}$ & $\overline{\mathcal{N}}_{\mathrm{C}_{2}\left(x_{3}\right)}^{0.6}$ & $\overline{\mathcal{N}}_{\mathrm{C}_{2}\left(x_{4}\right)}^{0.6}$ & $\overline{\mathcal{N}}_{\mathrm{C}_{2}\left(x_{5}\right)}^{0.6}$ \\
\hline$\left\{x_{1}, x_{2}, x_{3}\right\}$ & $\left\{x_{1}, x_{2}, x_{3}\right\}$ & $\left\{x_{3}\right\}$ & $\left\{x_{4}, x_{5}\right\}$ & $\left\{x_{5}\right\}$ \\
\hline
\end{tabular}

TAвle 7: $D_{\widehat{\mathrm{C}}_{1}}^{0.6}\left(x_{j}, x_{k}\right)(j, k=1,2, \ldots, 5)$.

\begin{tabular}{|c|c|c|c|c|c|}
\hline$U$ & $x_{1}$ & $x_{2}$ & $x_{3}$ & $x_{4}$ & $x_{5}$ \\
\hline$D_{\widehat{\mathrm{C}}_{1}}^{0.6}\left(x_{1}, x_{k}\right)$ & 1 & $1 / 5$ & $1 / 3$ & 0 & $1 / 3$ \\
\hline$D_{\widehat{\mathrm{C}}_{1}}^{0.6}\left(x_{2}, x_{k}\right)$ & $1 / 5$ & 1 & 0 & $1 / 3$ & $1 / 3$ \\
\hline$D_{\widehat{\mathrm{C}}_{1}}^{0.6}\left(x_{3}, x_{k}\right)$ & $1 / 3$ & 0 & 1 & 0 & 0 \\
\hline$D_{\widehat{C}_{1}}^{0.6}\left(x_{4}, x_{k}\right)$ & 0 & $1 / 3$ & 0 & 1 & 0 \\
\hline$D_{\widehat{\mathrm{C}}_{1}}^{0.6}\left(x_{5}, x_{k}\right)$ & $1 / 3$ & $1 / 3$ & 0 & 0 & 1 \\
\hline
\end{tabular}


TABLe 8: $D_{\widehat{\mathrm{C}}_{2}}^{0.6}\left(x_{j}, x_{k}\right)(j, k=1,2, \ldots, 5)$.

\begin{tabular}{|c|c|c|c|c|c|}
\hline$U$ & $x_{1}$ & $x_{2}$ & $x_{3}$ & $x_{4}$ & $x_{5}$ \\
\hline$D_{\widehat{\mathbf{C}}_{2}}^{0.6}\left(x_{1}, x_{k}\right)$ & 1 & 1 & $1 / 3$ & 0 & 0 \\
\hline$D_{\widehat{\mathrm{C}}_{2}}^{0.6}\left(x_{2}, x_{k}\right)$ & 1 & 1 & $1 / 3$ & 0 & 0 \\
\hline$D_{\widehat{\mathrm{C}}_{2}}^{0.6}\left(x_{3}, x_{k}\right)$ & $1 / 3$ & $1 / 3$ & 1 & 0 & 0 \\
\hline$D_{\widehat{\mathrm{C}}_{2}}^{0.6}\left(x_{4}, x_{k}\right)$ & 0 & 0 & 0 & 1 & $1 / 2$ \\
\hline$D_{\widehat{\mathrm{C}}_{2}}^{0.6}\left(x_{5}, x_{k}\right)$ & 0 & 0 & 0 & $1 / 2$ & 1 \\
\hline
\end{tabular}

$\left.\widehat{\mathrm{C}}_{i}(x y) \wedge A(y)\right\}=S \bar{R}_{\sum_{i=1}^{n} \widehat{\mathrm{C}}_{i}}(A)(x)$. On the other hand, $\wedge_{y \in U}\left\{\left(1-D_{\widehat{\mathrm{C}}_{i}}^{\beta}(x, y)\right) \vee A(y)\right\} \leq\left(1-D_{\widehat{\mathrm{C}}_{i}}^{\beta}(x, x)\right) \vee A(x)$ $=A(x)$. Hence, $\vee_{i=1}^{n} \wedge_{y \in U}\left\{\left(1-D_{\widehat{\mathrm{C}}_{i}}^{\beta}(x, y)\right) \vee A(y)\right\} \leq A$ $(x)$. So, $S \underline{R}_{\sum_{i=1}^{n}}^{{ }_{n} \widehat{C}_{i}}(A)(x) \leq A(x)$. Therefore, $S \underline{R}_{\sum_{i=1}^{n}}^{O} \widehat{\mathrm{C}}_{i}(A)$ $\subseteq A \subseteq S \bar{R}_{\sum_{i=1}^{n} \widehat{\mathrm{C}}_{i}}^{O}(A)$.

(2L) For any $x \in U$, if $A \subseteq B$, then $A(x) \leq B(x)$. Hence,

$$
\begin{aligned}
\underline{S R}_{\sum_{i=1}^{n} \widehat{\mathrm{C}}_{i}}^{O}(A)(x)= & \bigvee_{i=1}^{n} \bigwedge_{y \in U}\left\{\left(1-D_{\widehat{\mathrm{c}}_{i}}^{\beta}(x, y)\right) \bigvee A(y)\right\} \\
& \leq \bigvee_{i=1}^{n} \bigwedge_{y \in U}\left\{\left(1-D_{\widehat{\mathrm{c}}_{i}}^{\beta}(x, y)\right) \bigvee B(y)\right\} \\
= & S \underline{R}_{\sum_{i=1}^{O} \widehat{\mathrm{c}}_{i}}^{O}(B)(x),
\end{aligned}
$$

that is, $S \underline{R}_{\sum_{i=1}^{n}}^{O} \widehat{\mathrm{c}}_{i}(A) \subseteq S \underline{R}_{\sum_{i=1}^{n} \widehat{\mathrm{C}}_{i}}(B)$.

(2H) For any $x \in U$, if $A \subseteq B$, then $A(x) \leq B(x)$. Hence,

$$
\begin{aligned}
S \bar{R}_{\sum_{i=1}^{n} \widehat{\mathrm{C}}_{i}}^{O}(A)(x)= & \bigwedge_{i=1}^{n} \bigvee_{y \in U}\left\{D_{\widehat{\mathrm{C}}_{i}}^{\beta}(x, y) \bigwedge A(y)\right\} \\
& \leq \bigwedge_{i=1}^{n} \bigvee_{y \in U}\left\{D_{\widehat{\mathrm{C}}_{i}}^{\beta}(x, y) \bigwedge B(y)\right\} \\
& =S \bar{R}_{\sum_{i=1}^{n} \widehat{\mathrm{C}}_{i}}(B)(x),
\end{aligned}
$$

that is, $S \bar{R}_{\sum_{i=1}^{n} \widehat{\mathrm{C}}_{i}}(A) \subseteq S \bar{R}_{\sum_{i=1}^{n} \widehat{\mathrm{c}}_{i}}(B)$.

(3L) For each $x \in U$,

$$
\begin{aligned}
& S \underline{R}_{\sum_{i=1}^{n} \widehat{\mathbf{C}}_{i}}(A \bigcap B)(x)=\bigvee_{i=1}^{n} \bigwedge_{y \in U}\left\{\left(1-D_{\widehat{\mathbf{C}}_{i}}^{\beta}(x, y)\right) \bigvee(A \bigcap B)(y)\right\} \\
& =\bigvee_{i=1}^{n} \bigwedge_{y \in U}\left\{\left(\left(1-D_{\widehat{C}_{i}}^{\beta}(x, y)\right) \bigvee(A)(y)\right) \bigwedge\right. \\
& \left.\left(\left(1-D_{\widehat{\mathbf{C}}_{i}}^{\beta}(x, y)\right) \bigvee(B)(y)\right)\right\} \\
& =\left(\bigvee_{i=1}^{n} \bigwedge_{y \in U}\left\{\left(\left(-D_{\widehat{\mathrm{C}}_{i}}^{\beta}(x, y)\right) \bigvee(A)(y)\right\}\right) \wedge\left(\bigvee_{i=1}^{n} \bigwedge_{y \in U}\left\{\left(1-D_{\widehat{\mathrm{C}}_{i}}^{\beta}(x, y)\right) \bigvee(B)(y)\right\}\right)\right. \\
& =S \underline{R}_{\sum_{i=1}^{n} \widehat{\mathbf{C}}_{i}}(A)(x) \bigwedge S \underline{R}_{\sum_{i=1}^{n} \widehat{\mathbf{C}}_{i}}(B)(x) \\
& =\left(S \underline{R}_{\sum_{i=1}^{n}}^{O} \widehat{\mathbf{C}}_{i}(A) \bigcap S \underline{R}_{\sum_{i=1}^{n} \widehat{\mathbf{C}}_{i}}(B)\right)(x),
\end{aligned}
$$

that is, $S \underline{R}_{\sum_{i=1}^{O} \widehat{\mathbf{C}}_{i}}^{O}(A \cap B)=S \underline{R}_{\sum_{i=1}^{O} \widehat{\mathbf{C}}_{i}}(A) \cap S \underline{R}^{O} \sum_{i=1}^{n} \widehat{\mathbf{C}}_{i}(B)$.

Then, we only need to prove that $S \underline{R}_{\sum_{i=1}^{n}}^{O} \widehat{\mathrm{C}}_{i}(A \cup B) \supseteq$ $S \underline{R}_{\sum_{i=1}^{n}}^{O} \widehat{\mathrm{C}}_{i}(A) \cup S \underline{R}_{\sum_{i=1}^{n}}^{O} \widehat{\mathrm{C}}_{i}(B)$. According to (1L), we can

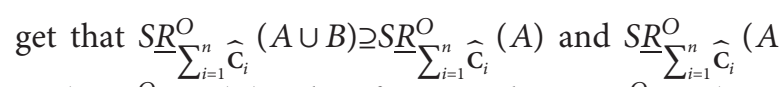
$\cup B) \supseteq S \underline{R}_{\sum_{i=1}^{n}}^{O} \hat{\mathrm{C}}_{i}(B)$. Therefore, we have $S \underline{R}_{\sum_{i=1}^{n}}^{O} \hat{\mathrm{C}}_{i}(A \cup$ $B) \supseteq S \underline{R}_{\sum_{i=1}^{n}}^{O \widehat{c}_{i}}(A) \cup S \underline{R}_{\sum_{i=1}^{n}}^{O} \widehat{\mathrm{c}}_{i}(B)$. 
(3H) For each $x \in U$,

$$
\begin{aligned}
& S \bar{R}_{\sum_{i=1}^{n} \widehat{\mathbf{C}}_{i}}(A \bigcup B)(x)=\bigwedge_{i=1}^{n} \bigvee_{y \in U}\left\{D_{\widehat{\mathbf{C}}_{i}}^{\beta}(x, y) \bigwedge(A \bigcup B)(y)\right\} \\
& =\bigwedge_{i=1}^{n} \bigvee_{y \in U}\left\{\left(D_{\widehat{\mathrm{C}}_{i}}^{\beta}(x, y) \bigwedge A(y)\right) \bigvee\left(D_{\widehat{\mathrm{C}}_{i}}^{\beta}(x, y) \bigwedge B(y)\right)\right\} \\
& =\left(\bigwedge_{i=1}^{n} \bigvee_{y \in U}\left\{D_{\widehat{\mathrm{C}}_{i}}^{\beta}(x, y) \bigwedge A(y)\right\}\right) \bigvee\left(\bigwedge_{i=1}^{n} \bigvee_{y \in U}\left\{D_{\widehat{\mathrm{C}}_{i}}^{\beta}(x, y) \wedge B(y)\right\}\right) \\
& =S \bar{R}_{\sum_{i=1}^{n} \widehat{\mathrm{C}}_{i}}(A)(x) \bigvee S \bar{R}_{\sum_{i=1}^{n} \widehat{\mathrm{C}}_{i}}(B)(x)=\left(S \bar{R}_{\sum_{i=1}^{n} \widehat{\mathrm{C}}_{i}}(A) \bigcup S \bar{R}_{\sum_{i=1}^{O} \widehat{\mathrm{C}}_{i}}(B)\right)(x) \text {, }
\end{aligned}
$$

that is, $S \bar{R}_{\sum_{i=1}^{n} \widehat{\mathrm{C}}_{i}}(A \cup B)=S \bar{R}_{\sum_{i=1}^{n} \widehat{\mathrm{C}}_{i}}(A) \cup S \bar{R}_{\sum_{i=1}^{n} \widehat{\mathrm{C}}_{i}}(B)$. Then, we only need to prove that $S \bar{R}_{\sum_{i=1}^{n} \widehat{\mathrm{C}}_{i}}(A \cap B) \subseteq$ $S \bar{R}_{\sum_{i=1}^{n} \widehat{\mathrm{C}}_{i}}(A) \cap S \bar{R}_{\sum_{i=1}^{n} \widehat{\mathrm{C}}_{i}}(B)$. According to (1H), we have $S \bar{R}_{\sum_{i=1}^{n} \widehat{\mathrm{C}}_{i}}^{O}(A \cap B) \subseteq S \bar{R}_{\sum_{i=1}^{n} \widehat{\mathrm{C}}_{i}}^{O}(A)$ and $S \bar{R}_{\sum_{i=1}^{n} \widehat{\mathrm{C}}_{i}}^{O}(A \cap B) \subseteq S$ $\bar{R}_{\sum_{i=1}^{n} \widehat{\mathrm{C}}_{i}}^{O}(B)$. Therefore, $S \bar{R}_{\sum_{i=1}^{n} \widehat{\mathrm{c}}_{i}}^{O}(A \cap B) \subseteq S \bar{R}_{\sum_{i=1}^{n} \widehat{\mathrm{C}}_{i}}^{O}(A) \cap$ $S \bar{R}_{\sum_{i=1}^{n} \widehat{C}_{i}}(B)$.

(4LH) For each $x \in U$,

$$
\begin{aligned}
S \underline{R}_{\sum_{i=1}^{n} \widehat{\mathrm{C}}_{i}}^{O}\left(A^{\prime}\right)(x) & =\bigvee_{i=1}^{n} \bigwedge_{y \in U}\left\{\left(1-D_{\widehat{\mathrm{C}}_{i}}^{\beta}(x, y)\right) \bigvee(1-A(y))\right\} \\
& =1-\bigwedge_{i=1}^{n} \bigvee_{y \in U}\left\{D_{\widehat{\mathrm{C}}_{i}}^{\beta}(x, y) \bigwedge A(y)\right\} \\
& =1-\bar{R}_{\sum_{i=1}^{n} \widehat{\mathrm{C}}_{i}}(A)(x) \\
& =\left(\bar{R}_{\sum_{i=1}^{O} \widehat{\mathrm{c}}_{i}}^{O}(A)\right)^{\prime}(x),
\end{aligned}
$$

that is, $\left(S \bar{R}_{\sum_{i=1}^{n} \widehat{\mathrm{C}}_{i}}(A)\right)^{\prime}=S \underline{R}_{\sum_{i=1}^{n} \widehat{\mathrm{C}}_{i}}\left(A^{\prime}\right)$. Then, it can be written as $S \underline{R}_{\sum_{i=1}^{O}}^{n} \widehat{\mathrm{C}}_{i}(A)=\left(S \bar{R}_{\sum_{i=1}^{n} \widehat{\mathrm{C}}_{i}}\left(A^{\prime}\right)\right)^{\prime}$. Therefore, $\left(S \underline{R}_{\sum_{i=1}^{n}}^{O} \widehat{\mathbf{c}}_{i}(A)\right)^{\prime}=S \bar{R}_{\sum_{i=1}^{n}}^{O} \widehat{\mathbf{c}}_{i}\left(A^{\prime}\right)$

Example 3 (continued from Example 1). By Example 2, $S \underline{R}_{\sum_{i=1}^{2} \widehat{\mathrm{C}}_{i}}(A)=\left(0.6667 / x_{1}\right)+\left(0.4 / x_{1}\right)+\left(0.6 / x_{1}\right)+\left(0.2 / x_{1}\right)$ $+\left(0.8 / x_{5}\right)$, and $S \bar{R}_{\sum_{i=1}^{2} \widehat{C}_{i}}(A)=\left(0.8 / x_{1}\right)+\left(0.4 / x_{2}\right)+(0.6 /$ $\left.x_{3}\right)+\left(0.333 / x_{4}\right)+\left(0.5 / x_{5}\right)$ for $A=\left(0.8 / x_{1}\right)+\left(0.4 / x_{2}\right)+$ $\left(0.6 / x_{3}\right)+\left(0.2 / x_{4}\right)+\left(0.5 / x_{5}\right)$. Let $B=\left(0.8 / x_{1}\right)+\left(0.5 / x_{2}\right)$ $+\left(0.7 / x_{3}\right)+\left(0.4 / x_{4}\right)+\left(0.6 / x_{5}\right)$ and $C=\left(0.9 / x_{1}\right)+(0.4 /$ $\left.x_{2}\right)+\left(0.3 / x_{3}\right)+\left(0.7 / x_{4}\right)+\left(0.8 / x_{5}\right)$. Then, $S \underline{R} \sum_{i=1}^{2} \widehat{\mathrm{c}}_{i}{ }^{O}(B)=$ $\left(0.6667 / x_{1}\right)+\left(0.5 / x_{2}\right)+\left(0.7 / x_{3}\right)+\left(0.4 / x_{4}\right)+\left(0.6 / x_{5}\right), \quad S$ $\bar{R}_{\sum_{i=1}^{2} \hat{\mathrm{c}}_{i}}(B)=\left(0.8 / x_{1}\right)+\left(0.5 / x_{2}\right)+\left(0.7 / x_{3}\right)+\left(0.4 / x_{4}\right)+$ $\left(0.6 / x_{5}\right), S \underline{R}_{\sum_{i=1}^{2} \widehat{\mathrm{C}}_{i}}^{O}(C)=\left(0.6667 / x_{1}\right)+\left(0.4 / x_{2}\right)+\left(0.3 / x_{3}\right)$ $+\left(0.7 / x_{4}\right)+\left(0.7 / x_{5}\right), \quad S \bar{R}_{\sum_{i=1}^{O} \widehat{\mathrm{C}}_{i}}(C)=\left(0.9 / x_{1}\right)+\left(0.4 / x_{2}\right)+$ $\left(0.333 / x_{3}\right)+\left(0.7 / x_{4}\right)+\left(0.8 / x_{5}\right), \quad S \underline{R}_{\sum_{i=1}^{O} \widehat{c}_{i}}(A \cup C)=$ $\left(0.6667 / x_{1}\right)+\left(0.4 / x_{2}\right)+\left(0.6 / x_{3}\right)+\left(0.7 / x_{4}\right)+\left(0.7 / x_{5}\right), \quad S$ $\bar{R}_{\sum_{i=1}^{O} \widehat{\mathrm{C}}_{i}}(A \cup C)=\left(0.9 / x_{1}\right)+\left(0.4 / x_{2}\right)+\left(0.6 / x_{3}\right)+\left(0.7 / x_{4}\right)$ $+\left(0.8 / x_{5}\right), S \underline{R}_{\sum_{i=1}^{O} \widehat{\mathrm{C}}_{i}}^{O}(A \cap C)=\left(0.6667 / x_{1}\right)+\left(0.4 / x_{2}\right)+(0.3 /$ $\left.x_{3}\right)+\left(0.2 / x_{4}\right)+\left(0.5 / x_{5}\right)$, and $S \bar{R}_{\sum_{i=1}^{2} \widehat{\mathrm{c}}_{i}}(A \cap C)=\left(0.8 / x_{1}\right)+$ $\left(0.4 / x_{2}\right)+\left(0.333 / x_{3}\right)+\left(0.333 / x_{4}\right)+\left(0.5 / x_{5}\right)$. Hence, the following hold:

(1LH) $S \underline{R}_{\sum_{i=1}^{O} \widehat{\mathbf{C}}_{i}}(A) \subseteq A \subseteq S \bar{R}_{\sum_{i=1}^{O} \widehat{\mathbf{C}}_{i}}(A)$, and the same applies for $B$ and $C$.

(2L) $S \underline{R}_{\sum_{i=1}^{2}}^{O} \widehat{\mathrm{C}}_{i}(A) \subseteq S \underline{R}_{\sum_{i=1}^{2} \widehat{\mathrm{C}}_{i}}^{O}(B)$.

(2H) $S \bar{R}_{\sum_{i=1}^{2}}^{O} \widehat{\mathbf{c}}_{i}(A) \subseteq S \bar{R}_{\sum_{i=1}^{O} \widehat{\mathbf{c}}_{i}}^{O}(B)$.

(3L) $\quad S \underline{R}_{\sum_{i=1}^{2} \widehat{\mathrm{c}}_{i}}^{O}(A \cap C)=S \underline{R}_{\sum_{i=1}^{2} \widehat{\mathrm{c}}_{i}}^{O}(A) \cap S \underline{R}_{\sum_{i=1}^{2} \widehat{\mathrm{c}}_{i}}(C)$, $S \underline{R}_{\sum_{i=1}^{O} \widehat{\mathrm{c}}_{i}}^{O}(A \cup C) \supseteq S \underline{R}_{\sum_{i=1}^{O} \widehat{\mathrm{c}}_{i}}^{O}(A) \cup S \underline{R}_{\sum_{i=1}^{2} \widehat{\mathrm{c}}_{i}}^{O}(C)$.

$(3 \mathrm{H}) \quad S \bar{R}_{\sum_{i=1}^{2} \widehat{\mathrm{C}}_{i}}(A \cap C) \subseteq S \bar{R}_{\sum_{i=1}^{O} \widehat{\mathrm{C}}_{i}}^{O}(A) \cap S \bar{R}_{\sum_{i=1}^{O} \widehat{\mathrm{C}}_{i}}^{O}(C)$, $S \bar{R}_{\sum_{i=1}^{2} \widehat{\mathrm{c}}_{i}}(A \cup C)=S \bar{R}_{\sum_{i=1}^{2} \widehat{\mathrm{c}}_{i}}(A) \cup S \bar{R}_{\sum_{i=1}^{2} \widehat{\mathrm{c}}_{i}}(C)$.

(4LH) Since $A=\left(0.8 / x_{1}\right)+\left(0.4 / x_{2}\right)+\left(0.6 / x_{3}\right)+(0.2 /$ $\left.x_{4}\right)+\left(0.5 / x_{5}\right), \quad A^{\prime}=\left(0.2 / x_{1}\right)+\left(0.6 / x_{2}\right)+\left(0.4 / x_{3}\right)+$ $\left(0.4 / x_{4}\right)+\left(0.5 / x_{5}\right)$. Hence, $S \underline{R}_{\sum_{i=1}^{O}}^{O} \widehat{\mathrm{c}}_{i}\left(A^{\prime}\right)=\left(0.2 / x_{1}\right)+$ $\left(0.6 / x_{2}\right)+\left(0.4 / x_{3}\right)+\left(0.6667 / x_{4}\right)+\left(0.5 / x_{5}\right), \quad$ and $S \bar{R}_{\sum_{i=1}^{2} \widehat{\mathrm{C}}_{i}}\left(A^{\prime}\right)=\left(0.333 / x_{1}\right)+\left(0.6 / x_{2}\right)+\left(0.4 / x_{3}\right)+(0.8 /$ $\left.x_{4}\right)+\left(0.5 / x_{5}\right)$. Therefore, $\quad\left(S \underline{R}_{\sum_{i=1}^{O} \widehat{\mathrm{C}}_{i}}(A)\right)^{\prime}=S \bar{R}_{\sum_{i=1}^{n}}^{O} \widehat{\mathrm{C}}_{i}$ $\left(A^{\prime}\right)$ and $\left(S \bar{R}_{\sum_{i=1}^{O} \widehat{\mathrm{C}}_{i}}(A)\right)^{\prime}=S \underline{R}_{\sum_{i=1}^{O}}^{O} \widehat{\mathrm{C}}_{i}\left(A^{\prime}\right)$.

Finally, the second type of covering-based optimistic multigranulation fuzzy rough sets is presented in Definition 6.

Definition 6. Let $\widetilde{\Gamma}=\left\{\widehat{\mathbf{C}}_{1}, \ldots, \widehat{\mathbf{C}}_{n}\right\}$ be an $n$-fuzzy $\beta$-coverings of $U$. For any $A \in F(U)$ and $\alpha \in[0,1)$, the optimistic 
multigranulation neighborhood fuzzy $\alpha$-lower approximation operator ( $\alpha$-OMNFLAO) $\underline{R}_{\sum_{i=1}^{O, \alpha} \widehat{\mathrm{C}}_{i}}(A)$ and optimistic multigranulation neighborhood fuzzy $\alpha$-upper approximation operator ( $\alpha$-OMNFUAO) $\bar{R}_{\sum_{i=1}^{n} \widehat{\mathrm{C}}_{i}}^{O, \alpha}(A)$ are defined as follows: for any $x \in U$,

$$
\begin{aligned}
& \underline{R}_{\sum_{i=1}^{n} \widehat{\mathbf{C}}_{i}}^{O, \alpha}(A)(x)=\bigvee_{i=1}^{n} \bigwedge_{y \in U}\left\{A(y): D_{\widehat{\mathbf{C}}_{i}}^{\beta}(x, y) \geq \alpha\right\}, \\
& \bar{R}_{\sum_{i=1}^{n, \alpha} \widehat{\mathbf{C}}_{i}}^{O, \alpha}(A)(x)=\bigwedge_{i=1}^{n} \bigvee_{y \in U}\left\{A(y): D_{\widehat{\mathbf{C}}_{i}}^{\beta}(x, y) \geq \alpha\right\} .
\end{aligned}
$$

In Definition 6, we call $A$ an $\alpha$-covering-based optimistic multigranulation neighborhood fuzzy rough set; otherwise, we call it an $\alpha$-covering-based optimistic multigranulation neighborhood definable set.

Example 4 (continued from Example 1). Let $\alpha=0.3$. Then, $\underline{R}_{\sum_{i=1}^{O} \widehat{C}_{i}, 0.3}^{2}(A)=\left(0.5 / x_{1}\right)+\left(0.4 / x_{2}\right)+\left(0.6 / x_{3}\right)+\left(0.2 / x_{4}\right)+(0.4$ $\left./ x_{5}\right)$ and $\bar{R}_{\sum_{i=1}^{2} \widehat{C}_{i}}^{O, 0.3}(A)=\left(0.8 / x_{1}\right)+\left(0.5 / x_{2}\right)+\left(0.8 / x_{3}\right)+(0.4 /$ $\left.x_{4}\right)+\left(0.5 / x_{5}\right)$.

Some characteristics of the $\alpha$-OMNFLAO $\underline{R}_{\sum_{i=1}^{O, \alpha} \widehat{\mathrm{C}}_{i}}^{{ }^{n}}(A)$ and the $\alpha$-OMNFUAO $\bar{R}_{\sum_{i=1}^{n} \widehat{C}_{i}}(A)$ are proposed in the following proposition.
Proposition 2. Let $\widetilde{\Gamma}=\left\{\widehat{\mathbf{C}}_{1}, \ldots, \widehat{\mathbf{C}}_{n}\right\}$ be an $n$-fuzzy $\beta$-coverings of $U$. Then, for any $A, B \in F(U)$ and $\alpha, \alpha_{1} \in[0,1)$, the following statements hold:

(1LH) $\underline{R} \sum_{i=1}^{O, \alpha} \widehat{\mathrm{C}}_{i}(A) \subseteq A \subseteq \bar{R}_{\sum_{i=1}^{O, \alpha} \widehat{\mathrm{C}}_{i}}(A)$

(2L) If $A \subseteq B$, then $\underline{R}_{\sum_{i=1}^{n, \alpha} \widehat{\mathrm{C}}_{i}}(A) \subseteq \underline{R}_{\sum_{i=1}^{n} \widehat{\mathrm{C}}_{i}}^{n, \alpha}(B)$

(2H) If $A \subseteq B$, then $\bar{R}_{\sum_{i=1}^{n, \alpha} \widehat{C}_{i}}(A) \subseteq \bar{R}_{\sum_{i=1}^{n} \widehat{C}_{i}, \alpha}(B)$

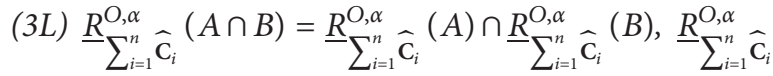

$(A \cup B) \supseteq \underline{R}_{\sum_{i=1}^{n, \alpha} \widehat{\mathrm{C}}_{i}}^{O,}(A) \cup \underline{R}_{\sum_{i=1}^{n} \widehat{\mathrm{C}}_{i}}^{O, \alpha}(B)$

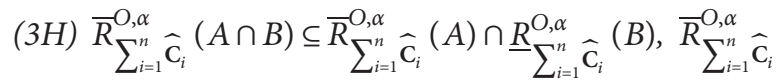

$(A \cup B)=\bar{R}_{\sum_{i=1}^{n} \widehat{\mathbf{C}}_{i}}^{O, \alpha}(A) \cup \bar{R}_{\sum_{i=1}^{n} \widehat{\mathbf{C}}_{i}}^{O, \alpha}(B)$

(4LH) $\left(\underline{R}_{\sum_{i=1}^{n, \alpha} \widehat{\mathrm{C}}_{i}}^{\mathrm{N}}(A)\right)^{\prime}=\bar{R}_{\sum_{i=1}^{\mathrm{O}, \alpha} \widehat{\mathrm{C}}_{i}}\left(A^{\prime}\right)$ and $\left(\bar{R}_{\sum_{i=1}^{n}, \widehat{\mathrm{C}}_{i}}^{n}(A)\right)^{\prime}$

$=\underline{R}_{\sum_{i=1}^{O, \alpha} \widehat{\mathbf{C}}_{i}}^{n}\left(A^{\prime}\right)$

(5LH) If $\alpha \leq \alpha_{1}$, then $\underline{R}_{\sum_{i=1}^{n} \widehat{\mathrm{C}}_{i}}^{O, \alpha}(A) \subseteq \underline{R}_{\sum_{i=1}^{n, \alpha_{1}} \widehat{\mathrm{C}}_{i}}(A)$ and $\bar{R}_{\sum_{i=1}^{n} \widehat{\mathbf{C}}_{i}}^{O, \alpha}(A) \supseteq \bar{R}_{\sum_{i=1}^{n} \widehat{\mathbf{C}}_{i}}^{\alpha_{1}} \widehat{C}^{(A)}$

Proof. (1LH) For any $x \in U$,

$$
\begin{aligned}
\underline{R}_{\sum_{i=1}^{n} \widehat{\mathbf{C}}_{i}}^{O, \alpha}(A)(x) & =\bigvee_{i=1}^{n} \bigwedge_{y \in U}\left\{A(y): D_{\widehat{\mathbf{C}}_{i}}^{\beta}(x, y) \geq \alpha\right\} \leq \bigvee_{i=1}^{n} A(x)=A(x) \\
& \leq \bigwedge_{i=1}^{n} \bigvee_{y \in U}\left\{A(y): D_{\widehat{\mathbf{C}}_{i}}^{\beta}(x, y) \geq \alpha\right\}=\bar{R}_{\sum_{i=1}^{n} \widehat{\mathbf{C}}_{i}}^{O, \alpha}(A)(x) .
\end{aligned}
$$

(2L) For any $x \in U$, if $A \subseteq B$, then $A(x) \leq B(x)$. Hence,

$$
\begin{aligned}
\underline{R}_{\sum_{i=1}^{n}}^{O, \alpha} \widehat{\mathrm{C}}_{i}(A)(x) & =\bigvee_{i=1}^{n} \bigwedge_{y \in U}\left\{A(y): D_{\widehat{\mathrm{C}}_{i}}^{\beta}(x, y) \geq \alpha\right\} \\
& \leq \bigvee_{i=1}^{n} \bigvee_{y \in U}\left\{B(y): D_{\widehat{\mathrm{C}}_{i}}^{\beta}(x, y) \geq \alpha\right\} \\
& =\underline{R}_{\sum_{i=1}^{O} \widehat{\mathrm{C}}_{i}}^{{ }_{n}}(B)(x),
\end{aligned}
$$

$$
\begin{aligned}
\bar{R}_{\sum_{i=1}^{n} \widehat{\mathbf{C}}_{i}}^{O, \alpha}(A)(x) & =\bigwedge_{i=1}^{n} \bigvee_{y \in U}\left\{A(y): D_{\widehat{\mathrm{C}}_{i}}^{\beta}(x, y) \geq \alpha\right\} \\
& \leq \bigwedge_{i=1}^{n} \bigvee_{y \in U}\left\{B(y): D_{\widehat{\mathrm{C}}_{i}}^{\beta}(x, y) \geq \alpha\right\} \\
& =\bar{R}_{\sum_{i=1}^{O, \alpha} \widehat{\mathbf{C}}_{i}}^{n_{i}}(B)(x),
\end{aligned}
$$

that is, $\bar{R}_{\sum_{i=1}^{n} \widehat{\mathrm{C}}_{i}, \alpha}^{n}(A) \subseteq \bar{R}_{\sum_{i=1}^{n} \widehat{\mathrm{C}}_{i}}^{\mathrm{O}, \alpha}(B)$.

that is, $\underline{R}_{\sum_{i=1}^{n} \widehat{\mathrm{C}}_{i}}^{O, \alpha}(A) \subseteq \underline{R}_{\sum_{i=1}^{n} \widehat{\mathrm{C}}_{i}}^{\mathrm{n} \alpha}(B)$.

(2H) For any $x \in U$, if $A \subseteq B$, then $A(x) \leq B(x)$. Hence,
(3L) For each $x \in U$,

$$
\underline{R}_{\sum_{i=1}^{n} \widehat{\mathbf{C}}_{i}}^{O, \alpha}(A \bigcap B)(x)=\bigvee_{i=1}^{n} \bigwedge_{y \in U}\left\{(A \bigcap B)(y): D_{\widehat{\mathbf{C}}_{i}}^{\beta}(x, y) \geq \alpha\right\}
$$




$$
\begin{aligned}
& =\bigvee_{i=1}^{n} \bigwedge_{y \in U}\left\{A(y) \wedge B(y): D_{\widehat{\mathbf{C}}_{i}}^{\beta}(x, y) \geq \alpha\right\} \\
& =\left(\bigvee_{i=1}^{n} \bigwedge_{y \in U}\left\{A(y): D_{\widehat{\mathrm{c}}_{i}}^{\beta}(x, y) \geq \alpha\right\}\right) \wedge\left(\bigvee_{i=1}^{n} \bigwedge_{y \in U}\left\{B(y): D_{\widehat{\mathrm{c}}_{i}}^{\beta}(x, y) \geq \alpha\right\}\right) \\
& =\underline{R}_{\sum_{i=1}^{n}, \widehat{\mathrm{c}}_{i}}^{O, \alpha}(A)(x) \wedge \underline{R}_{\sum_{i=1}^{O, \alpha} \widehat{\mathrm{c}}_{i}}^{(B)(x)} \\
& =\left(\underline{R}^{O, \alpha} \sum_{i=1}^{n} \widehat{\mathbf{C}}_{i}(A) \bigcap \underline{R}_{\sum_{i=1}^{n} \widehat{\mathbf{C}}_{i}}^{O, \alpha}(B)\right)(x),
\end{aligned}
$$

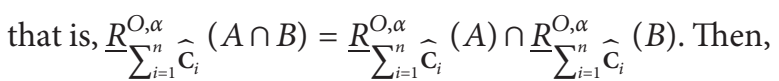
we only need to prove that $\underline{R}_{\sum_{i=1}^{n}, \alpha} \widehat{\mathrm{c}}_{i}(A \cup B) \supseteq \underline{R} \underline{R}_{\sum_{i=1}^{n} \widehat{\mathrm{C}}_{i}}^{O, \alpha}(A)$ $\cup \underline{R^{O, \alpha}} \sum_{i=1}^{n} \widehat{\mathrm{C}}_{i}(B)$. According to (2L), we have $\underline{R}_{\sum_{i=1}^{n} \widehat{\mathrm{C}}_{i}}^{O, \alpha}$
$(A \cup B) \supseteq \underline{R}_{\sum_{i=1}^{O, \alpha} \widehat{\mathrm{c}}_{i}}^{(A)}$ and $\quad \underline{R}_{\sum_{i=1}^{n}, \widehat{\mathrm{c}}_{i}}^{O, \alpha}(A \cup B) \supseteq \underline{R}_{\sum_{i=1}^{O, \alpha} \widehat{\mathrm{c}}_{i}}^{O,}(B)$. Therefore, $\underline{R}_{\sum_{i=1}^{n} \widehat{\mathrm{C}}_{i}}^{O, \alpha}(A \cup B) \supseteq \underline{\underline{R}} \underline{\sum}_{\sum_{i=1}^{n} \widehat{\mathrm{C}}_{i}}^{O, \alpha}(A) \cup \underline{R}_{\sum_{i=1}^{n} \widehat{\mathrm{C}}_{i}}^{O, \alpha}(B)$. $(3 \mathrm{H})$ The proof is similar to $(3 \mathrm{~L})$. Hence, we omit it. (4LH) For each $x \in U$,

$$
\begin{aligned}
\underline{R}_{\sum_{i=1}^{O, \alpha} \widehat{\mathbf{C}}_{i}}^{\mathrm{\alpha}}\left(A^{\prime}\right)(x) & =\bigvee_{i=1}^{n} \bigwedge_{y \in U}\left\{A^{\prime}(y): D_{\widehat{\mathbf{C}}_{i}}^{\beta}(x, y) \geq \alpha\right\} \\
& =\bigvee_{i=1}^{n} \bigwedge_{y \in U}\left\{1-A(y): D_{\widehat{\mathrm{C}}_{i}}^{\beta}(x, y) \geq \alpha\right\} \\
& =1-\bigwedge_{i=1}^{n} \bigvee_{y \in U}\left\{A(y): D_{\widehat{\mathbf{C}}_{i}}^{\beta}(x, y) \geq \alpha\right\}=\left(\bar{R}_{\sum_{i=1}^{O, \alpha} \widehat{\mathbf{C}}_{i}}(A)\right)^{\prime}(x),
\end{aligned}
$$

that is, $\left(\bar{R}_{\sum_{i=1}^{n}, \widehat{\mathrm{C}}_{i}}^{O, \alpha}(A)\right)^{\prime}=\underline{R}_{\sum_{i=1}^{O, \alpha} \widehat{\mathrm{C}}_{i}}^{O,}\left(A^{\prime}\right)$. Then, it can be written as $\underline{R}_{\sum_{i=1}^{n} \widehat{\mathbf{c}}_{i}}^{O, \alpha}(A)=\left(\bar{R}_{\sum_{i=1}^{O, \alpha} \widehat{\mathbf{c}}_{i}}^{O, \alpha}\left(A^{\prime}\right)\right)^{\prime}$. Therefore, $\left(\underline{R}^{O, \alpha} \sum_{i=1}^{n} \widehat{\mathrm{c}}_{i}(A)\right)^{\prime}=\bar{R}_{\sum_{i=1}^{n, \alpha} \widehat{\mathrm{c}}_{i}}^{\left({ }^{\prime}\right.}\left(A^{\prime}\right)$.

$(5 \mathrm{LH})$ It is immediate by Definition 6.

Example 5 (continued from Example 1). In Example 4, we have $\underline{R}_{\sum_{i=1}^{O}}^{O, 0.3} \widehat{\mathrm{c}}_{i}(A)=\left(0.5 / x_{1}\right)+\left(0.4 / x_{2}\right)+\left(0.6 / x_{3}\right)+\left(0.2 / x_{4}\right)+$ $\left(0.4 / x_{5}\right)$ and $\bar{R}_{\sum_{i=1}^{2} \widehat{c}_{i}}^{O, 0.3}(A)=\left(0.8 / x_{1}\right)+\left(0.5 / x_{2}\right)+\left(0.8 / x_{3}\right)+$ $\left(0.2 / x_{4}\right)+\left(0.5 / x_{5}\right)$. Let $B=\left(0.8 / x_{1}\right)+\left(0.5 / x_{1}\right)+\left(0.7 / x_{1}\right)+$ $\left(0.4 / x_{1}\right)+\left(0.6 / x_{5}\right)$ and $C=\left(0.9 / x_{1}\right)+\left(0.4 / x_{1}\right)+(0.3 /$ $\left.x_{1}\right)+\left(0.7 / x_{1}\right)+\left(0.8 / x_{5}\right)$. Then, $S \underline{S}_{\sum_{i=1}^{O}, 0.3}^{2} \widehat{\mathrm{c}}_{i}(B)=\left(0.6 / x_{1}\right)+$ $\left(0.5 / x_{2}\right)+\left(0.7 / x_{3}\right)+\left(0.4 / x_{4}\right)+\left(0.5 / x_{5}\right), S \bar{R}_{\sum_{i=1}^{O}, 0.3}^{2} \widehat{\mathrm{c}}_{i}(B)=(0.8$ $\left./ x_{1}\right)+\left(0.6 / x_{2}\right)+\left(0.8 / x_{3}\right)+\left(0.5 / x_{4}\right)+\left(0.6 / x_{5}\right), \quad S \underline{R}^{O, 0.3} \sum_{i=1}^{2} \widehat{\mathrm{c}}_{i}$ $(C)=\left(0.3 / x_{1}\right)+\left(0.4 / x_{2}\right)+\left(0.3 / x_{3}\right)+\left(0.7 / x_{4}\right)+\left(0.7 / x_{5}\right)$, $S \bar{R}_{\sum_{i=1}^{O}, \widehat{C}_{i}}^{O, 0.3}(C)=\left(0.9 / x_{1}\right)+\left(0.8 / x_{2}\right)+\left(0.9 / x_{3}\right)+\left(0.7 / x_{4}\right)+$ $\left(0.8 / x_{5}\right), S \underline{R}_{\sum_{i=1}^{O}, 0.3}^{\mathrm{C}_{i}}(A \cup C)=\left(0.6 / x_{1}\right)+\left(0.4 / x_{2}\right)+\left(0.6 / x_{3}\right)+$ $\left(0.7 / x_{4}\right)+\left(0.7 / x_{5}\right), S \bar{R}_{\sum_{i=1}^{O}, 0.3}^{2} \widehat{\mathrm{c}}_{i}(A \cup C)=\left(0.9 / x_{1}\right)+\left(0.8 / x_{2}\right)+$ $\left(0.9 / x_{3}\right)+\left(0.7 / x_{4}\right)+\left(0.8 / x_{5}\right), S \underline{R}_{\sum_{i=1}^{O, 0.3}}^{2} \widehat{\mathrm{c}}_{i}(A \cap C)=\left(0.3 / x_{1}\right)+$ $\left(0.3 / x_{2}\right)+\left(0.3 / x_{3}\right)+\left(0.2 / x_{4}\right)+\left(0.4 / x_{5}\right)$, and $S \bar{R}_{\sum_{i=1}^{O}, 0.3}^{2} \widehat{\mathrm{c}}_{i}(A \cap$ $C)=\left(0.8 / x_{1}\right)+\left(0.5 / x_{2}\right)+\left(0.8 / x_{3}\right)+\left(0.4 / x_{4}\right)+\left(0.5 / x_{5}\right)$.

Hence, the following hold:

(1LH) $\frac{R}{\sum_{i=1}^{2,0.3} \widehat{\mathrm{c}}_{i}}(A) \subseteq A \subseteq \bar{R}_{\sum_{i=1}^{O, 0.3} \widehat{\mathrm{c}}_{i}}(A)$, and the same applies for $B$ and $C$

(2L) $\underline{R}_{\sum_{i=1}^{O} \widehat{\mathbf{c}}_{i}}^{O, 0.3}(A) \subseteq \underline{R}_{\sum_{i=1}^{O}, 0.3}^{2} \widehat{\mathrm{c}}_{i}(B)$

(2H) $\bar{R}_{\sum_{i=1}^{O}, 0.3}^{2} \widehat{\mathrm{c}}_{i}(A) \subseteq \bar{R}_{\sum_{i=1}^{O}, 0.3}^{2} \widehat{\mathrm{c}}_{i}(B)$



$(A \cup C) \supseteq \underline{R}_{\sum_{i=1}^{O}, \widehat{\mathrm{c}}_{i}}^{O, .3}(A) \cup \underline{R}_{\sum_{i=1}^{O, 0.3} \widehat{\mathrm{c}}_{i}}^{2}(C)$

$(3 \mathrm{H}) \bar{R}_{\sum_{i=1}^{2}, \widehat{\mathrm{c}}_{i}}^{2,0.3}(A \cap C)=\bar{R}_{\sum_{i=1}^{O}, 0.3}^{2} \widehat{\mathrm{c}}_{i}(A) \cap \bar{R}_{\sum_{i=1}^{2}, 0.3}^{2} \widehat{\mathrm{c}}_{i}(C), \bar{R}_{\sum_{i=1}^{2}}^{O, 0.3} \widehat{\mathrm{c}}_{i}$ $(A \cup C) \supseteq \bar{R}_{\sum_{i=1}^{O}, \widehat{\mathrm{C}}_{i}}^{2,0.3}(A) \cup \bar{R}_{\sum_{i=1}^{O, 0.3} \widehat{\mathrm{c}}_{i}}^{2}(C)$

(4LH) Since $\underline{R}_{\sum_{i=1}^{O}, 0.3}^{2} \widehat{\mathrm{c}}_{i}\left(A^{\prime}\right)=\left(0.2 / x_{1}\right)+\left(0.5 / x_{2}\right)+(0.2 /$ $\left.x_{3}\right)+\left(0.6 / x_{4}\right)+\left(0.5 / x_{5}\right)$ and $\bar{R}_{\sum_{i=1}^{O}, 0.3}^{2} \widehat{\mathrm{c}}_{i}\left(A^{\prime}\right)=\left(0.5 / x_{1}\right)+$ 
$\left(0.6 / x_{2}\right)+\left(0.4 / x_{3}\right)+\left(0.8 / x_{4}\right)+\left(0.6 / x_{5}\right)$, then $\left(\underline{R}^{O, 0.3} \sum_{i=1}^{2} \widehat{\mathrm{C}}\right.$

$(A))^{\prime}=\bar{R}_{\sum_{i=1}^{n} \widehat{\mathrm{C}}_{i}}^{O, 0.3}\left(A^{\prime}\right)$ and $\left(\bar{R}_{\sum_{i=1}^{O} \widehat{\mathrm{C}}_{i}}^{2,0.3}(A)\right)^{\prime}=\underline{R}_{\sum_{i=1}^{n} \widehat{\mathrm{C}}_{i}}^{O, 0.3}\left(A^{\prime}\right)$

(5LH) Since $\underline{R}_{\sum_{i=1}^{2} \widehat{C}_{i}}^{O, 0.3}\left(A^{\prime}\right)=\left(0.5 / x_{1}\right)+\left(0.4 / x_{2}\right)+(0.6 /$

$\left.x_{3}\right)+\left(0.2 / x_{4}\right)+\left(0.4 / x_{5}\right)$ and $\bar{R}_{\sum_{i=1}^{2}, 0.3}^{2} \widehat{\mathrm{c}}_{i}\left(A^{\prime}\right)=\left(0.8 / x_{1}\right)+$

$\left(0.5 / x_{2}\right)+\left(0.8 / x_{3}\right)+\left(0.4 / x_{4}\right)+\left(0.5 / x_{5}\right), \quad$ then

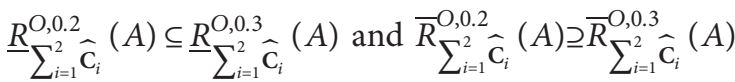

3.2. Covering-Based Pessimistic Multigranulation Fuzzy Rough Sets. Based on the covering-based optimistic multigranulation fuzzy rough sets in Section 3.1, we present two types of covering-based pessimistic multigranulation fuzzy rough sets in this section. Firstly, the first type is presented in Definition 7.

Definition 7. Let $\widetilde{\Gamma}=\left\{\widehat{\mathbf{C}}_{1}, \ldots, \widehat{\mathbf{C}}_{n}\right\}$ be an $n$-fuzzy $\beta$-coverings of $U$. For any $A \in F(U)$, the pessimistic multigranulation neighborhood fuzzy lower approximation operator (PMNFLAO) $S \underline{R}_{\sum_{i=1}^{P}}^{P} \widehat{\mathrm{C}}_{i}(A)$ and the pessimistic multigranulation neighborhood fuzzy upper approximation operator (PMNFUAO) $S \bar{R}_{\sum_{i=1}^{n} \widehat{\mathrm{C}}_{i}}(A)$ are defined as follows: for any $x \in U$,

$$
\begin{aligned}
& S \underline{R}_{\sum_{i=1}^{n} \widehat{\mathbf{C}}_{i}}^{P}(A)(x)=\bigwedge_{i=1}^{n} \bigwedge_{y \in U}\left\{\left(1-D_{\widehat{\mathbf{C}}_{i}}^{\beta}(x, y)\right) \bigvee A(y)\right\}, \\
& S \bar{R}_{\sum_{i=1}^{n} \widehat{\mathbf{C}}_{i}}^{P}(A)(x)=\bigvee_{i=1}^{n} \bigvee_{y \in U}\left\{D_{\widehat{\mathrm{C}}_{i}}^{\beta}(x, y) \bigwedge A(y)\right\} .
\end{aligned}
$$

In Definition 7, we call $A$ a covering-based pessimistic multigranulation neighborhood fuzzy rough set; otherwise, we call it a covering-based pessimistic multigranulation neighborhood definable set.

Example 6 (continued from Example 1). $S \underline{R}_{\sum_{i=1}^{P} \widehat{\mathrm{C}}_{i}}^{P}(A)=$ $\left(0.4 / x_{1}\right)+\left(0.4 / x_{2}\right)+\left(0.6 / x_{3}\right)+\left(0.2 / x_{4}\right)+\left(0.5 / x_{5}\right), S \bar{R}_{\sum_{i=1}^{2} \widehat{\mathrm{c}}_{i}}^{P}$ $(A)=\left(0.8 / x_{1}\right)+\left(0.8 / x_{2}\right)+\left(0.6 / x_{3}\right)+\left(0.5 / x_{4}\right)+\left(0.5 / x_{5}\right)$.

Some characteristics of the PMNFLAO $S \underline{R}_{\sum_{i=1}^{P}}^{P} \widehat{\mathrm{c}}_{i}(A)$ and the PMNFUAO $S \bar{R}_{\sum_{i=1}^{n} \widehat{\mathrm{C}}_{i}}^{P}(A)$ are presented in Proposition 3.

Proposition 3. Let $\widetilde{\Gamma}=\left\{\widehat{\mathbf{C}}_{1}, \ldots, \widehat{\mathbf{C}}_{n}\right\}$ be an $n$-fuzzy $\beta$-coverings of $U$. Then, for any $A, B \in F(U)$, we have the following statements:

$$
\text { (1LH) } S \underline{R}_{\sum_{i=1}^{n}}^{P} \widehat{\mathrm{C}}_{i}(A) \subseteq A \subseteq S \bar{R}_{\sum_{i=1}^{n} \widehat{\mathrm{C}}_{i}}^{(A)}
$$

(2L) If $A \subseteq B$, then $S \underline{R}_{\sum_{i=1}^{n} \widehat{\mathrm{c}}_{i}}(A) \subseteq S \underline{R}_{\sum_{i=1}^{P}}^{P} \widehat{\mathrm{c}}_{i}(B)$

(2H) If $A \subseteq B$, then $S \bar{R}_{\sum_{i=1}^{n} \widehat{\mathrm{c}}_{i}}(A) \subseteq S \bar{R}_{\sum_{i=1}^{P} \widehat{\mathrm{c}}_{i}}(B)$

(3L) $\quad \underline{S R}_{\sum_{i=1}^{n} \widehat{\mathrm{c}}_{i}}(A \cap B)=S \underline{R}_{\sum_{i=1}^{n} \widehat{\mathrm{c}}_{i}}^{P}(A) \cap S \underline{R}_{\sum_{i=1}^{n} \widehat{\mathrm{c}}_{i}}^{P}(B)$,

$S \underline{R}_{\sum_{i=1}^{n} \widehat{\mathrm{c}}_{i}}^{P}(A \cup B) \supseteq \underline{S} \underline{R}_{\sum_{i=1}^{n} \widehat{\mathrm{c}}_{i}}(A) \cup S \underline{R}_{\sum_{i=1}^{n} \widehat{\mathrm{c}}_{i}}^{(B)}$

(3H) $\quad S \bar{R}_{\sum_{i=1}^{n} \widehat{\mathrm{c}}_{i}}^{P}(A \cap B) \subseteq S \bar{R}_{\sum_{i=1}^{n} \widehat{\mathrm{c}}_{i}}^{P}(A) \cap S \bar{R}_{\sum_{i=1}^{n} \widehat{\mathrm{c}}_{i}}^{P}(B)$,

$S \bar{R}_{\sum_{i=1}^{n} \widehat{\mathbf{c}}_{i}}(A \cup B)=S \bar{R}_{\sum_{i=1}^{n} \widehat{\mathrm{c}}_{i}}^{P}(A) \cup S \bar{R}_{\sum_{i=1}^{n} \widehat{\mathbf{c}}_{i}}(B)$

(4LH) $\quad\left(S \underline{R}_{\sum_{i=1}^{n} \widehat{\mathrm{c}}_{i}}(A)\right)^{\prime}=S \bar{R}_{\sum_{i=1}^{n} \widehat{\mathrm{c}}_{i}}\left(A^{\prime}\right), \quad\left(S \bar{R}_{\sum_{i=1}^{n} \widehat{\mathrm{c}}_{i}}(A)\right)^{\prime}$

$=S \underline{R}_{\sum_{i=1}^{n} \widehat{\mathbf{C}}_{i}}\left(A^{\prime}\right)$

Proof

(1LH) For any $x \in U$ and $i=1,2, \ldots, n, D_{\widehat{\mathbf{C}}_{i}}^{\beta}(x, x)=1$ by Definition 4 . Then, $\left.A(x)=A(x) \wedge D_{\widehat{C}_{i}}^{\beta} \stackrel{\mathrm{C}_{i}}{x}, x\right) \leq \mathrm{V}_{y \in U}$ $\left\{D_{\widehat{\mathbf{C}}_{i}}^{\beta}(x, y) \wedge A(y)\right\}$. Hence, $A(x) \leq \vee_{i=1}^{n} \vee_{y \in U}\left\{D_{\widehat{\mathbf{C}}_{i}}^{\beta}(x\right.$, $y) \wedge A \quad(y)\}=S \bar{R}_{\sum_{i=1}^{P} \widehat{\mathrm{C}}_{i}}(A)(x)$. On the other hand, $\wedge_{y \in U}\left\{\left(1-D_{\widehat{\mathbf{C}}_{i}}^{\beta}(x, y)\right) \vee A(y)\right\} \quad \leq\left(1-D_{\widehat{\mathbf{C}}_{i}}^{\beta}(x, x)\right) \vee$ $A(x)=A(x)$. Hence, $\wedge_{i=1}^{n} \wedge \wedge_{y \in U}\left\{\left(1-D_{\widehat{\mathrm{C}}_{i}}^{\beta}(x, y)\right) \vee A(y)\right\}$ $\leq A(x)$. So, we have $S \underline{R}^{P} \sum_{i=1}^{n} \widehat{\mathrm{C}}_{i}(A)(x) \leq A(x)$. Therefore, $S \underline{R}^{P} \sum_{i=1}^{n} \widehat{\mathrm{C}}_{i}(A) \subseteq A \subseteq S \bar{R}_{\sum_{i=1}^{P} \widehat{\mathrm{C}}_{i}}(A)$.

(2L) For any $x \in U$, if $A \subseteq B$, then $A(x) \leq B(x)$. Hence,

$$
\begin{aligned}
& S \underline{R}_{\sum_{i=1}^{n} \widehat{\mathbf{C}}_{i}}^{P}(A)(x)=\bigwedge_{i=1}^{n} \bigwedge_{y \in U}\left\{\left(1-D_{\widehat{\mathbf{C}}_{i}}^{\beta}(x, y)\right) \bigvee A(y)\right\} \\
& \leq \bigwedge_{i=1}^{n} \bigwedge_{y \in U}\left\{\left(1-D_{\widehat{\mathrm{C}}_{i}}^{\beta}(x, y)\right) \bigvee B(y)\right\} \\
& =S \underline{R}_{\sum_{i=1}^{n} \widehat{\mathrm{C}}_{i}}^{P}(B)(x) \text {, }
\end{aligned}
$$

that is, $\underline{S R}_{\sum_{i=1}^{n}}^{P} \widehat{\mathrm{C}}_{i}(A) \subseteq S \underline{R}_{\sum_{i=1}^{n} \widehat{\mathrm{C}}_{i}}^{P}(B)$.

(2H) For any $x \in U$, if $A \subseteq B$, then $A(x) \leq B(x)$. Hence,

$$
\begin{aligned}
& S \bar{R}_{\sum_{i=1}^{n} \widehat{\mathbf{c}}_{i}}^{P}(A)(x)=\bigvee_{i=1}^{n} \bigvee_{y \in U}\left\{D_{\widehat{\mathrm{C}}_{i}}^{\beta}(x, y) \wedge A(y)\right\} \\
\leq & \bigvee_{i=1}^{n} \bigvee_{y \in U}\left\{D_{\widehat{\mathrm{C}}_{i}}^{\beta}(x, y) \bigwedge B(y)\right\}=S \bar{R}_{\sum_{i=1}^{n} \widehat{\mathbf{c}}_{i}}^{P}(B)(x),
\end{aligned}
$$

that is, $S \bar{R}_{\sum_{i=1}^{n} \widehat{\mathrm{C}}_{i}}^{P}(A) \subseteq S \bar{R}_{\sum_{i=1}^{n} \widehat{\mathrm{C}}_{i}}(B)$.

(3L) For each $x \in U$, 


$$
\begin{aligned}
S \underline{R}_{\sum_{i=1}^{n} \widehat{\mathbf{c}}_{i}}^{P}(A \bigcap B)(x) & =\bigwedge_{i=1}^{n} \bigwedge_{y \in U}\left\{\left(1-D_{\widehat{\mathbf{C}}_{i}}^{\beta}(x, y)\right) \bigvee(A \bigcap B)(y)\right\} \\
& =\bigwedge_{i=1}^{n} \bigwedge_{y \in U}\left\{\left(\left(1-D_{\widehat{\mathbf{C}}_{i}}^{\beta}(x, y)\right) \bigvee(A)(y)\right) \wedge\left(\left(1-D_{\widehat{\mathbf{C}}_{i}}^{\beta}(x, y)\right) \bigvee(B)(y)\right)\right\} \\
& =\left(\bigwedge_{i=1}^{n} \bigwedge_{y \in U}\left\{\left(1-D_{\widehat{\mathbf{C}}_{i}}^{\beta}(x, y)\right) \bigvee(A)(y)\right\}\right) \wedge\left(\bigwedge_{i=1}^{n} \bigwedge_{y \in U}\left\{\left(1-D_{\widehat{\mathrm{C}}_{i}}^{\beta}(x, y)\right) \bigvee(B)(y)\right\}\right) \\
& =S \underline{R}_{\sum_{i=1}^{n} \widehat{\mathbf{c}}_{i}}(A)(x) \bigwedge S \underline{R}_{\sum_{i=1}^{n} \widehat{\mathbf{c}}_{i}}^{P}(B)(x) \\
& =\left(S \underline{R}_{\sum_{i=1}^{n} \widehat{\mathbf{c}}_{i}}(A) \bigcap S \underline{R}_{\sum_{i=1}^{n} \widehat{\mathbf{c}}_{i}}^{(B)}\right)(x),
\end{aligned}
$$

that is, $S \underline{R}_{\sum_{i=1}^{n} \widehat{\mathrm{c}}_{i}}^{P}(A \cap B)=S \underline{R}_{\sum_{i=1}^{n} \widehat{\mathrm{c}}_{i}}^{P}(A) \cap S \underline{R}_{\sum_{i=1}^{n} \widehat{\mathrm{c}}_{i}}(B)$. Then, we only need to prove that $S \underline{R}_{\sum_{i=1}^{n}}^{P} \widehat{\mathrm{C}}_{i}(A \cup B)$


have $S \underline{R}_{\sum_{i=1}^{n} \widehat{\mathrm{c}}_{i}}^{P}(A \cup B) \supseteq S \underline{R}_{\sum_{i=1}^{n} \widehat{\mathrm{c}}_{i}}^{P}(A)$ and also have

$S \underline{R}_{\sum_{i=1}^{n} \widehat{\mathbf{c}}_{i}}^{P}(A \cup B) \supseteq S \underline{R}_{\sum_{i=1}^{n} \widehat{\mathbf{c}}_{i}}^{P}(B)$. Therefore, there is $S \underline{R}_{\sum_{i=1}^{P} \widehat{\mathrm{c}}_{i}}^{P}(A \cup B) \supseteq S \underline{R}_{\sum_{i=1}^{P} \widehat{\mathrm{c}}_{i}}^{P}(A) \cup S \underline{R}_{\sum_{i=1}^{n} \widehat{\mathrm{c}}_{i}}^{P}(B)$.

(3H) For each $x \in U$,

$$
\begin{aligned}
S \bar{R}_{\sum_{i=1}^{n} \widehat{\mathbf{c}}_{i}}^{P}(A \bigcup B)(x) & =\bigvee_{i=1}^{n} \bigvee_{y \in U}\left\{D_{\widehat{\mathbf{c}}_{i}}^{\beta}(x, y) \wedge(A \bigcup B)(y)\right\} \\
& =\bigvee_{i=1}^{n} \bigvee_{y \in U}\left\{\left(D_{\widehat{\mathbf{c}}_{i}}^{\beta}(x, y) \wedge A(y)\right) \bigvee\left(D_{\widehat{\mathbf{c}}_{i}}^{\beta}(x, y) \wedge B(y)\right)\right\} \\
& =\left(\bigvee_{i=1}^{n} \bigvee_{y \in U}\left\{D_{\widehat{\mathbf{c}}_{i}}^{\beta}(x, y) \wedge A(y)\right\}\right) \bigvee\left(\bigvee_{i=1}^{n} \bigvee_{y \in U}\left\{D_{\widehat{\mathbf{c}}_{i}}^{\beta}(x, y) \wedge B(y)\right\}\right) \\
& =S \bar{R}_{\sum_{i=1}^{n} \widehat{\mathbf{c}}_{i}}(A)(x) \bigvee S \bar{R}_{\sum_{i=1}^{n} \widehat{\mathbf{c}}_{i}}^{P}(B)(x)=\left(S \bar{R}_{\sum_{i=1}^{n} \widehat{\mathbf{c}}_{i}}^{P}(A) \bigcup S \bar{R}_{\sum_{i=1}^{n} \widehat{\mathbf{c}}_{i}}^{P}(B)\right)(x),
\end{aligned}
$$

that is, $S \bar{R}_{\sum_{i=1}^{n} \widehat{\mathrm{c}}_{i}}^{P}(A \cup B)=S \bar{R}_{\sum_{i=1}^{n} \widehat{\mathrm{c}}_{i}}(A) \cup S \bar{R}_{\sum_{i=1}^{n} \widehat{\mathrm{c}}_{i}}(B)$. Then, we only need to prove that $S \bar{R}_{\sum_{i=1}^{n} \widehat{\mathrm{C}}_{i}}(A \cap B)$ $\subseteq S \bar{R}_{\sum_{i=1}^{n} \widehat{\mathrm{c}}_{i}}^{P}(A) \cap S \bar{R}_{\sum_{i=1}^{n} \widehat{\mathrm{c}}_{i}}^{P}(B)$. According to (1H), we have $\quad S \bar{R}_{\sum_{i=1}^{n} \widehat{\mathrm{c}}_{i}}^{P}(A \cap B) \subseteq S \bar{R}_{\sum_{i=1}^{n} \widehat{\mathrm{c}}_{i}}^{P}(A) \quad$ and $S \bar{R}_{\sum_{i=1}^{n} \widehat{\mathrm{C}}_{i}}^{P}(A \cap B) \quad \subseteq S \bar{R}_{\sum_{i=1}^{n} \widehat{\mathrm{C}}_{i}}^{(B)}$. Therefore, $S \bar{R}_{\sum_{i=1}^{n} \widehat{\mathrm{c}}_{i}}^{P}(A \cap B) \subseteq S \bar{R}_{\sum_{i=1}^{n} \widehat{\mathrm{c}}_{i}}^{P}(A) \cap S \bar{R}_{\sum_{i=1}^{n} \widehat{\mathrm{c}}_{i}}(B)$.

(4LH) For each $x \in U$,

$$
\begin{aligned}
& S \underline{R}^{P} \sum_{i=1}^{n} \widehat{\mathrm{c}}_{i}\left(A^{\prime}\right)(x)=\bigwedge_{i=1}^{n} \bigwedge_{y \in U}\left\{\left(1-D_{\widehat{\mathrm{C}}_{i}}^{\beta}(x, y)\right) \bigvee(1-A(y))\right\} \\
& =1-\bigvee_{i=1}^{n} \bigvee_{y \in U}\left\{D_{\widehat{\mathbf{C}}_{i}}^{\beta}(x, y) \wedge A(y)\right\} \\
& =1-\bar{R}_{\sum_{i=1}^{n} \widehat{\mathrm{C}}_{i}}(A)(x) \\
& =\left(\bar{R}_{\sum_{i=1}^{n} \widehat{\mathbf{c}}_{i}}(A)\right)^{\prime}(x) \text {, }
\end{aligned}
$$

that is, $\left(S \bar{R}_{\sum_{i=1}^{n}}^{P} \widehat{\mathrm{c}}_{i}(A)\right)^{\prime}=S \underline{R}_{\sum_{i=1}^{n} \widehat{\mathrm{c}}_{i}}\left(A^{\prime}\right)$. Then, it can be written as $S \underline{R}_{\sum_{i=1}^{n} \widehat{\mathrm{c}}_{i}}^{P}(A)=\left(\hat{S R}_{\sum_{i=1}^{n} \widehat{\mathrm{c}}_{i}}\left(A^{\prime}\right)\right)^{\prime}$. Therefore, $\left(S \underline{R}_{\sum_{i=1}^{n}}^{P} \widehat{\mathrm{c}}_{i}(A)\right)^{\prime}=S \bar{R}_{\sum_{i=1}^{n} \widehat{\mathrm{c}}_{i}}\left(A^{\prime}\right)$.

Example 7 (continued from Example 1). By Example 6, $S \underline{R}_{\sum_{i=1}^{2} \widehat{\mathrm{C}}_{i}}^{P}(A)=\left(0.4 / x_{1}\right)+\left(0.4 / x_{2}\right)+\left(0.6 / x_{3}\right)+\left(0.2 / x_{4}\right)+$ $\left(0.5 / x_{5}\right), S \bar{R}_{\sum_{i=1}^{2} \widehat{\mathrm{c}}_{i}}^{P}(A)=\left(0.8 / x_{1}\right)+\left(0.8 / x_{2}\right)+\left(0.6 / x_{3}\right)+(0.5 /$ $\left.x_{4}\right)+\left(0.5 / x_{5}\right)$. Let $\left(0.8 / x_{1}\right)+\left(0.5 / x_{2}\right)+\left(0.7 / x_{3}\right)+\left(0.4 / x_{4}\right)+$ $\left(0.6 / x_{5}\right)$ and $C=\left(0.9 / x_{1}\right)+\left(0.4 / x_{2}\right)+\left(0.3 / x_{3}\right)+\left(0.7 / x_{4}\right)+$ $\left(0.8 / x_{5}\right)$. Then, $S \underline{R}_{\sum_{i=1}^{P} \widehat{\mathrm{c}}_{i}}^{P}(B)=\left(0.5 / x_{1}\right)+\left(0.5 / x_{2}\right)+(0.6667$ $\left./ x_{3}\right)+\left(0.4 / x_{4}\right)+\left(0.5 / x_{5}\right), S \bar{R}_{\sum_{i=1}^{2} \widehat{\mathrm{c}}_{i}}^{P}(B)=\left(0.8 / x_{1}\right)+\left(0.8 / x_{2}\right)$ $+\left(0.7 / x_{3}\right)+\left(0.5 / x_{4}\right)+\left(0.6 / x_{5}\right), \quad S \underline{R}_{\sum_{i=1}^{2} \widehat{\mathrm{c}}_{i}}^{P}(C)=\quad(0.6667 /$ $\left.x_{1}\right)+\left(0.4 / x_{2}\right)+\left(0.3 / x_{3}\right)+\left(0.7 / x_{4}\right)+\left(0.7 / x_{5}\right), S \bar{R}_{\sum_{i=1}^{2} \widehat{\mathrm{c}}_{i}}^{P}(C)=$ $\left(0.9 / x_{1}\right)+\left(0.9 / x_{2}\right)+\left(0.333 / x_{3}\right)+\left(0.7 / x_{4}\right)+\left(0.8 / x_{5}\right)$, $S \underline{R}_{\sum_{i=1}^{P} \widehat{\mathrm{c}}_{i}}^{P}(A \cup C)=\left(0.4 / x_{1}\right)+\left(0.4 / x_{2}\right)+\left(0.6 / x_{3}\right)+(0.6667 /$ 
$\left.x_{4}\right)+\left(0.6667 / x_{5}\right), \quad S \bar{R}_{\sum_{i=1}^{2} \widehat{\mathrm{c}}_{i}}^{P}(A \cup C)=\left(0.9 / x_{1}\right)+\left(0.9 / x_{2}\right)+$ $\left(0.6 / x_{3}\right)+\left(0.7 / x_{4}\right)+\left(0.8 / x_{5}\right), \quad S \underline{R}_{\sum_{i=1}^{2} \widehat{\mathrm{C}}_{i}}^{P}(A \cap C)=\left(0.4 / x_{1}\right)$ $+\left(0.4 / x_{2}\right)+\left(0.3 / x_{3}\right)+\left(0.2 / x_{4}\right)+\left(0.5 / x_{5}\right)$, and $S \bar{R}_{\sum_{i=1}^{2} \widehat{c}_{i}}(A \cap$ $C)=\left(0.8 / x_{1}\right)+\left(0.8 / x_{2}\right)+\left(0.333 / x_{3}\right)+\left(0.5 / x_{4}\right)+\left(0.5 / x_{5}\right)$.

Hence, the following hold:

(1LH) $S \underline{R}_{\sum_{i=1}^{2} \widehat{\mathrm{C}}_{i}}^{P}(A) \subseteq A \subseteq S \bar{R}_{\sum_{i=1}^{2} \widehat{\mathrm{C}}_{i}}(A)$, and the same applies for $B$ and $C$

(2L) $S \underline{R}_{\sum_{i=1}^{P} \hat{\mathrm{c}}_{i}}(A) \subseteq S \underline{R}_{\sum_{i=1}^{P} \hat{\mathrm{c}}_{i}}^{(B)}$

(2H) $S \bar{R}_{\sum_{i=1}^{2} \widehat{\mathrm{c}}_{i}}(A) \subseteq S \bar{R}_{\sum_{i=1}^{P} \widehat{\mathrm{c}}_{i}}(B)$

(3L) $\quad S \underline{R}_{\sum_{i=1}^{2} \widehat{\mathrm{c}}_{i}}^{P}(A \cap C)=S \underline{R}_{\sum_{i=1}^{2} \widehat{\mathrm{c}}_{i}}(A) \cap S \underline{R}_{\sum_{i=1}^{P} \hat{\mathrm{c}}_{i}}(C)$, $S \underline{R}_{\sum_{i=1}^{2} \widehat{\mathrm{c}}_{i}}(A \cup C) \supseteq S \underline{R}_{\sum_{i=1}^{2} \widehat{\mathrm{c}}_{i}}(A) \cup S \underline{R}_{\sum_{i=1}^{2} \widehat{\mathrm{c}}_{i}}^{(C)}$

(3H) $\quad S \bar{R}_{\sum_{i=1}^{2} \widehat{\mathrm{c}}_{i}}^{P}(A \cap C) \subseteq S \bar{R}_{\sum_{i=1}^{2} \widehat{\mathrm{c}}_{i}}^{P}(A) \cap S \bar{R}_{\sum_{i=1}^{2} \widehat{\mathrm{c}}_{i}}^{P}(C)$, $S \bar{R}_{\sum_{i=1}^{2} \widehat{\mathrm{c}}_{i}}^{P}(A \cup C)=S \bar{R}_{\sum_{i=1}^{2} \widehat{\mathrm{c}}_{i}}^{P}(A) \cup S \bar{R}_{\sum_{i=1}^{2} \widehat{\mathrm{c}}_{i}}^{(C)}$

(4LH) Since $\quad S \underline{R}_{\sum_{i=1}^{P}}^{P} \widehat{\mathrm{c}}_{i}\left(A^{\prime}\right)=\left(0.2 / x_{1}\right)+\left(0.2 / x_{2}\right)+$ $\left(0.4 / x_{3}\right)+\left(0.5 / x_{4}\right)+\left(0.5 / x_{5}\right), \quad S \bar{R}_{\sum_{i=1}^{2} \widehat{\mathrm{c}}_{i}}\left(A^{\prime}\right)=(0.6 /$ $\left.x_{1}\right)+\left(0.6 / x_{2}\right)+\left(0.4 / x_{3}\right)+\left(0.8 / x_{4}\right)+\left(0.5 / x_{5}\right)$, then $\left(S \underline{R}_{\sum_{i=1}^{P} \widehat{\mathrm{c}}_{i}}^{P}(A)\right)^{\prime}=S \bar{R}_{\sum_{i=1}^{n} \widehat{\mathrm{c}}_{i}}^{P}\left(A^{\prime}\right) \quad$ and $\quad\left(S \bar{R}_{\sum_{i=1}^{2} \widehat{\mathrm{c}}_{i}}(A)\right)^{\prime}$ $=S \underline{R}_{\sum_{i=1}^{P}}^{P} \widehat{\mathrm{c}}_{i}\left(A^{\prime}\right)$

Finally, the second type of covering-based pessimistic multigranulation fuzzy rough set model is presented in Definition 8.

Definition 8. Let $\widetilde{\Gamma}=\left\{\widehat{\mathbf{C}}_{1}, \ldots, \widehat{\mathbf{C}}_{n}\right\}$ be an $n$-fuzzy $\beta$-coverings of $U$. For any $A \in F(U)$ and $\alpha \in[0,1]$, the pessimistic multigranulation neighborhood fuzzy $\alpha$-lower approximation operator ( $\alpha$-PMNFLAO) $\underline{R}_{\sum_{i=1}^{P, \alpha} \widehat{\mathrm{c}}_{i}}(A)$ and the pessimistic multigranulation neighborhood fuzzy $\alpha$-upper approximation operator ( $\alpha$-PMNFUAO) $\bar{R}_{\sum_{i=1}^{n} \widehat{c}_{i}}^{P} \widehat{c}(A)$ are defined as follows: for any $x \in U$,

$$
\begin{aligned}
& \underline{R}_{\sum_{i=1}^{n} \widehat{\mathbf{c}}_{i}}^{P, \alpha}(A)(x)=\bigwedge_{i=1}^{n} \bigwedge_{y \in U}\left\{A(y): D_{\widehat{\mathrm{C}}_{i}}^{\beta}(x, y) \geq \alpha\right\}, \\
& \bar{R}_{\sum_{i=1}^{P, \alpha} \widehat{\mathbf{C}}_{i}}^{P,}(A)(x)=\bigvee_{i=1}^{n} \bigvee_{y \in U}\left\{A(y): D_{\widehat{\mathrm{C}}_{i}}^{\beta}(x, y) \geq \alpha\right\} .
\end{aligned}
$$

In Definition 8 , we call $A$ an $\alpha$-covering-based pessimistic multigranulation neighborhood fuzzy rough set; otherwise, we call it an $\alpha$-covering-based pessimistic multigranulation neighborhood definable set.

Example 8 (continued from Example 1). Let $\alpha=0.3$. Then, $\underline{R^{R}} \sum_{i=1}^{P, 0.3} \widehat{\mathrm{C}}_{i}(A)=\left(0.4 / x_{1}\right)+\left(0.2 / x_{2}\right)+\left(0.4 / x_{3}\right)+\left(0.2 / x_{4}\right)+$ $\left(0.2 / x_{5}\right)$ and $\bar{R}_{\sum_{i=1}^{2} \widehat{\mathrm{C}}_{i}}^{P, 0.3}(A)=\left(0.8 / x_{1}\right)+\left(0.8 / x_{2}\right)+\left(0.8 / x_{3}\right)+$ $\left(0.5 / x_{4}\right)+\left(0.8 / x_{5}\right)$.

Some characteristics of the $\alpha$-PMNFLAO $\underline{R}_{\sum_{i=1}^{P}, \alpha, \widehat{\mathrm{C}}_{i}}(A)$ and the $\alpha$-PMNFUAO $\bar{R}_{\sum_{i=1}^{n}, \alpha} \widehat{\mathrm{C}}_{i}(A)$ are proposed in the following proposition.

Proposition 4. Let $\widetilde{\Gamma}=\left\{\widehat{\mathbf{C}}_{1}, \ldots, \widehat{\mathbf{C}}_{n}\right\}$ be an $n$-fuzzy $\beta$-coverings of $U$. Then, for any $A, B \in F(U)$ and $\alpha, \alpha_{1} \in[0,1)$, the following statements hold:

(1LH) $\underline{R}_{\sum_{i=1}^{n} \widehat{\mathrm{c}}_{i}}^{P, \alpha}(A) \subseteq A \subseteq \bar{R}_{\sum_{i=1}^{P, \alpha} \widehat{\mathrm{c}}_{i}}(A)$

(2L) If $A \subseteq B$, then $\underline{R}_{\sum_{i=1}^{n} \widehat{\mathrm{c}}_{i}}^{P, \alpha}(A) \subseteq \underline{R}_{\sum_{i=1}^{P, \alpha} \widehat{\mathrm{c}}_{i}}(B)$

(2H) If $A \subseteq B$, then $\bar{R}_{\sum_{i=1}^{P}, \alpha}^{{ }_{1}} \widehat{\mathrm{c}}_{i}(A) \subseteq \bar{R}_{\sum_{i=1}^{P, \alpha} \widehat{\mathrm{c}}_{i}}(B)$

(3L) $\bar{R}_{\sum_{i=1}^{P}, \alpha}^{n} \widehat{\mathrm{C}}_{i}(A \cap B)=\bar{R}_{\sum_{i=1}^{n} \widehat{\mathrm{c}}_{i}}^{P, \alpha}(A) \cap \underline{R}_{\sum_{i=1}^{n} \widehat{\mathrm{C}}_{i}}^{P, \alpha}(B), \bar{R}_{\sum_{i=1}^{n}, \alpha}^{\hat{\mathrm{C}}_{i}}$

$(A \cup B) \supseteq \bar{R}_{\sum_{i=1}^{P, \alpha} \widehat{\mathrm{c}}_{i}}^{P,}(A) \cup \underline{R}_{\sum_{i=1}^{P, \alpha} \widehat{\mathrm{c}}_{i}}^{(B)}$

(3H) $\bar{R}_{\sum_{i=1}^{n} \widehat{\mathrm{C}}_{i}}^{P, \alpha}(A \cap B) \subseteq \bar{R}_{\sum_{i=1}^{n} \widehat{\mathrm{c}}_{i}}^{P, \alpha}(A) \cap \bar{R}_{\sum_{i=1}^{n} \widehat{\mathrm{c}}_{i}}^{P, \alpha}(B), \bar{R}_{\sum_{i=1}^{n} \widehat{\mathrm{c}}_{i}}^{P, \alpha}$

$(A \cup B)=\bar{R}_{\sum_{i=1}^{n} \widehat{\mathrm{C}}_{i}}^{P, \alpha}(A) \cup \bar{R}_{\sum_{i=1}^{P, \alpha} \widehat{\mathrm{C}}_{i}}(B)$

(4LH) $\quad\left(\underline{R}_{\sum_{i=1}^{n, \alpha} \widehat{\mathrm{c}}_{i}}^{P}(A)\right)^{\prime}=\bar{R}_{\sum_{i=1}^{P, \alpha} \widehat{\mathrm{c}}_{i}}^{P}\left(A^{\prime}\right), \quad\left(\bar{R}_{\sum_{i=1}^{P, \alpha} \widehat{\mathrm{c}}_{i}}(A)\right)^{\prime}=$ $\underline{R} \sum_{i=1}^{P, \alpha} \widehat{\mathrm{c}}_{i}^{n}\left(A^{\prime}\right)$

(5LH) If $\alpha \leq \alpha_{1}$, then $\underline{R}_{\sum_{i=1}^{n}, \widehat{\mathrm{c}}_{i}}^{(, \alpha) \subseteq \underline{R}}{\underline{\sum_{i=1}^{n} \widehat{\mathrm{c}}_{i}}}^{P, \alpha_{1}}(A)$ and $\bar{R}_{\sum_{i=1}^{P, \alpha} \widehat{\mathbf{c}}_{i}}(A) \supseteq \bar{R}_{\sum_{i=1}^{P, \alpha_{1}} \widehat{\mathbf{c}}_{i}}^{(A)}$

Proof

(1LH) For any $x \in U$,

$$
\begin{aligned}
& \underline{R}_{\sum_{i=1}^{n} \widehat{\mathrm{C}}_{i}}^{P, \alpha}(A)(x)=\bigwedge_{i=1}^{n} \bigwedge_{y \in U}\left\{A(y): D_{\widehat{\mathrm{C}}_{i}}^{\beta}(x, y) \geq \alpha\right\} \\
& \leq \bigwedge_{i=1}^{n} A(x)=A(x) \\
& \leq \bigvee_{i=1}^{n} \bigvee_{y \in U}\left\{A(y): D_{\widehat{\mathrm{C}}_{i}}^{\beta}(x, y) \geq \alpha\right\} \\
& =\bar{R}_{\sum_{i=1}^{n}, \alpha}^{\mathbf{c}_{i}}(A)(x) \text {. }
\end{aligned}
$$

(2L) For any $x \in U$, if $A \subseteq B$, then $A(x) \leq B(x)$. Hence,

$$
\begin{aligned}
& \underline{R}^{P, \alpha} \sum_{i=1}^{n} \widehat{\mathrm{C}}_{i}(A)(x)=\bigwedge_{i=1}^{n} \bigwedge_{y \in U}\left\{A(y): D_{\widehat{\mathrm{C}}_{i}}^{\beta}(x, y) \geq \alpha\right\} \\
& \leq \bigwedge_{i=1}^{n} \bigwedge_{y \in U}\left\{B(y): D_{\widehat{\mathrm{c}}_{i}}^{\beta}(x, y) \geq \alpha\right\} \\
& =\bar{R}_{\sum_{i=1}^{n}, \alpha}^{\mathbf{c}_{i}}(B)(x),
\end{aligned}
$$

that is, $\underline{R}_{\sum_{i=1}^{n} \widehat{\mathrm{c}}_{i}}^{P, \alpha}(A) \subseteq \underline{R}_{\sum_{i=1}^{P, \alpha} \widehat{\mathrm{c}}_{i}}(B)$. 
(2H) For any $x \in U$, if $A \subseteq B$, then $A(x) \leq B(x)$. Hence,

$$
\begin{aligned}
\bar{R}_{\sum_{i=1}^{n} \widehat{\mathbf{c}}_{i}}^{P, \alpha}(A)(x) & =\bigvee_{i=1}^{n} \bigvee_{y \in U}\left\{A(y): D_{\widehat{\mathbf{C}}_{i}}^{\beta}(x, y) \geq \alpha\right\} \\
& \leq \bigvee_{i=1}^{n} \bigvee_{y \in U}\left\{B(y): D_{\widehat{\mathbf{C}}_{i}}^{\beta}(x, y) \geq \alpha\right\} \\
& =\bar{R}_{\sum_{i=1}^{P, \alpha} \widehat{\mathbf{c}}_{i}}(B)(x),
\end{aligned}
$$

that is, $\bar{R}_{\sum_{i=1}^{n}, \alpha}^{\widehat{C}_{i}}(A) \subseteq \bar{R}_{\sum_{i=1}^{n}{ }^{P, \alpha} \widehat{\mathbf{c}}_{i}}(B)$.

(3L) For each $x \in U$,

$$
\begin{aligned}
\underline{R}_{\sum_{i=1}^{n} \widehat{\mathbf{C}}_{i}}^{P, \alpha}(A \bigcap B)(x)= & \bigwedge_{i=1}^{n} \bigwedge_{y \in U}\left\{(A \bigcap B)(y): D_{\widehat{\mathbf{C}}_{i}}^{\beta}(x, y) \geq \alpha\right\} \\
= & \bigwedge_{i=1}^{n} \bigwedge_{y \in U}\left\{A(y) \bigwedge B(y): D_{\widehat{\mathbf{C}}_{i}}^{\beta}(x, y) \geq \alpha\right\} \\
= & \left(\bigwedge_{i=1}^{n} \bigwedge_{y \in U}\left\{A(y): D_{\widehat{\mathbf{C}}_{i}}^{\beta}(x, y) \geq \alpha\right\}\right) \bigwedge \\
& \cdot\left(\bigwedge_{i=1}^{n} \bigwedge_{y \in U}\left\{B(y): D_{\widehat{\mathbf{C}}_{i}}^{\beta}(x, y) \geq \alpha\right\}\right) \\
= & \underline{R}_{\sum_{i=1}^{n} \widehat{\mathbf{C}}_{i}}^{P, \alpha}(A)(x) \bigwedge \underline{R}^{P, \alpha} \sum_{i=1}^{n} \widehat{\mathbf{C}}_{i}(B)(x)=\left(\underline{R}_{\sum_{i=1}^{n, \alpha} \widehat{\mathbf{C}}_{i}}(A) \bigcap \underline{R^{R}} \sum_{i=1}^{P, \alpha} \widehat{\mathbf{C}}_{i}(B)\right)(x),
\end{aligned}
$$

that is, $\underline{R}_{\sum_{i=1}^{P, \alpha} \widehat{\mathbf{C}}_{i}}(A \cap B)=\underline{R}^{P, \alpha} \sum_{i=1}^{n} \widehat{\mathbf{C}}_{i}(A) \cap \underline{R}^{P, \alpha} \sum_{i=1}^{n} \widehat{\mathbf{C}}_{i}(B)$. Then, we only need to prove that $\underline{R}_{\sum_{i=1}^{n}, \widehat{\mathrm{C}}_{i}}^{P, \alpha}(A \cup B) \supseteq \underline{R}^{P, \alpha} \sum_{i=1}^{n} \widehat{\mathrm{C}}_{i}$ $(A) \cup \underline{R}_{\sum_{i=1}^{n, \alpha} \widehat{\mathrm{C}}_{i}}^{P}(B)$. According to (2L), we have $\underline{R}^{P, \alpha} \sum_{i=1}^{n} \widehat{\mathbf{C}}_{i}(A \cup B) \supseteq \underline{R}^{P, \alpha} \sum_{i=1}^{n} \widehat{\mathbf{C}}_{i}(A) \quad$ and $\quad \underline{R^{P, \alpha}} \sum_{i=1}^{n} \widehat{\mathbf{C}}_{i}(A \cup B) \supseteq$
$\underline{R}_{\sum_{i=1}^{n, \alpha} \widehat{\mathbf{C}}_{i}}(B)$. Therefore, we get that $\underline{R}_{\sum_{i=1}^{P, \alpha} \widehat{\mathbf{C}}_{i}}(A \cup B)$ $\supseteq \underline{R}_{\sum_{i=1}^{n} \widehat{\mathrm{C}}_{i}}^{P, \alpha}(A) \cup \underline{R}_{\sum_{i=1}^{n, \alpha} \widehat{\mathrm{C}}_{i}}^{(B)}$.

$(3 \mathrm{H})$ The proof is similar to $(3 \mathrm{~L})$. Hence, we omit it. (4LH) For each $x \in U$,

$$
\begin{aligned}
\underline{R}_{\sum_{i=1}^{n} \widehat{\mathbf{C}}_{i}}^{P, \alpha}\left(A^{\prime}\right)(x) & =\bigwedge_{i=1}^{n} \bigwedge_{y \in U}\left\{A^{\prime}(y): D_{\widehat{\mathbf{C}}_{i}}^{\beta}(x, y) \geq \alpha\right\} \\
& =\bigwedge_{i=1}^{n} \bigwedge_{y \in U}\left\{1-A(y): D_{\widehat{\mathbf{c}}_{i}}^{\beta}(x, y) \geq \alpha\right\} \\
& =1-\bigvee_{i=1}^{n} \bigvee_{y \in U}\left\{A(y): D_{\widehat{\mathbf{c}}_{i}}^{\beta}(x, y) \geq \alpha\right\}=\left(\bar{R}_{\sum_{i=1}^{n} \widehat{\mathbf{c}}_{i}}^{P}(A)\right)^{\prime}(x),
\end{aligned}
$$

that is, $\left(\bar{R}_{\sum_{i=1}^{n, \alpha} \widehat{\mathrm{C}}_{i}}(A)\right)^{\prime}=\underline{R}_{\sum_{i=1}^{P, \alpha} \widehat{\mathrm{C}}_{i}}^{P}\left(A^{\prime}\right)$. Then, it can be written as $\underline{R}_{\sum_{i=1}^{n}}^{P, \alpha} \widehat{\mathrm{C}}_{i}(A)=\left(\bar{R}_{\sum_{i=1}^{n} \widehat{\mathrm{C}}_{i}}^{P, \alpha}\left(A^{\prime}\right)\right)^{\prime}$. Therefore, $\left(\underline{R}_{\sum_{i=1}^{P, \alpha} \widehat{\mathbf{C}}_{i}}^{P}(A)\right)^{\prime}=\bar{R}_{\sum_{i=1}^{n}{ }^{n}, \alpha}^{\hat{\mathbf{C}}_{i}}\left(A^{\prime}\right)$.

(5LH) It is immediate by Definition 8 .

Example 9 (continued from Example 1). In Example 8, we have $\underline{R}_{\sum_{i=1}^{P}{ }^{2} \widehat{\mathbf{C}}_{i}}^{2}(A)=\left(0.4 / x_{1}\right)+\left(0.2 / x_{2}\right)+\left(0.4 / x_{3}\right)+\left(0.2 / x_{4}\right)$ $+\left(0.2 / x_{5}\right)$ and $\bar{R}_{\sum_{i=1}^{P}, 0.3}^{2} \widehat{\mathrm{C}}_{i}(A)=\left(0.8 / x_{1}\right)+\left(0.8 / x_{2}\right)+\left(0.8 / x_{3}\right)+$ $\left(0.5 / x_{4}\right)+\left(0.8 / x_{5}\right)$. Let $B=\left(0.8 / x_{1}\right)+\left(0.5 / x_{1}\right)+\left(0.7 / x_{1}\right)$ $+\left(0.4 / x_{1}\right)+\left(0.6 / x_{5}\right)$ and $C=\left(0.9 / x_{1}\right)+\left(0.4 / x_{1}\right)+\left(0.3 / x_{1}\right)$ $+\left(0.7 / x_{1}\right)+\left(0.8 / x_{5}\right)$. Then, $S \underline{R}_{\sum_{i=1}^{P, 0.3} \widehat{\mathrm{C}}_{i}}^{2}(B)=\left(0.5 / x_{1}\right)+(0.4 /$ $\left.x_{2}\right)+\left(0.5 / x_{3}\right)+\left(0.4 / x_{4}\right)+\left(0.4 / x_{5}\right), \quad S \bar{R}_{\sum_{i=1}^{P}, 0.3}^{2} \widehat{\mathrm{C}}_{i}(B)=(0.8 /$ $\left.x_{1}\right)+\left(0.8 / x_{2}\right)+\left(0.8 / x_{3}\right)+\left(0.6 / x_{4}\right)+\left(0.8 / x_{5}\right), \quad S \underline{R}^{P, 0.3} \sum_{i=1}^{2} \widehat{\mathrm{C}}_{i}$ $(C)=\left(0.5 / x_{1}\right)+\left(0.4 / x_{2}\right)+\left(0.5 / x_{3}\right)+\left(0.4 / x_{4}\right)+\left(0.4 / x_{5}\right)$, $S \bar{R}_{\sum_{i=1}^{2} \widehat{C}_{i}, 0.3}^{2}(C)=\left(0.9 / x_{1}\right)+\left(0.9 / x_{2}\right)+\left(0.9 / x_{3}\right)+\left(0.8 / x_{4}\right)+$ 
$\left(0.9 / x_{5}\right), \quad S \underline{R}_{\sum_{i=1}^{P, 0.3}}^{\sum_{1}} \widehat{\mathrm{c}}_{i}(A \cup C)=\left(0.4 / x_{1}\right)+\left(0.4 / x_{2}\right)+\left(0.4 / x_{3}\right)$ $+\left(0.4 / x_{4}\right)+\left(0.4 / x_{5}\right), S \bar{R}_{\sum_{i=1}^{2,0.3} \widehat{\mathrm{c}}_{i}}^{P}(A \cup C)=\left(0.9 / x_{1}\right)+\left(0.9 / x_{2}\right)$ $+\left(0.9 / x_{3}\right)+\left(0.8 / x_{4}\right)+\left(0.9 / x_{5}\right), S \underline{R}_{\sum_{i=1}^{P}}^{P, 0.3} \widehat{\mathrm{c}}_{i}(A \cap C)=\left(0.3 / x_{1}\right)$ $+\left(0.2 / x_{2}\right)+\left(0.3 / x_{3}\right)+\left(0.2 / x_{4}\right)+\left(0.2 / x_{5}\right)$, and $S \bar{R}_{\sum_{i=1}^{2}, 0.3}^{2} \widehat{\mathbf{c}}_{i}$ $(A \cap C)=\left(0.8 / x_{1}\right)+\left(0.8 / x_{2}\right)+\left(0.8 / x_{3}\right)+\left(0.5 / x_{4}\right)+(0.8 /$ $\left.x_{5}\right)$. Hence, the following hold:

(1LH) $\underline{R}_{\sum_{i=1}^{P}, 0.3}^{2} \widehat{\mathrm{c}}_{i}(A) \subseteq A \subseteq \bar{R}_{\sum_{i=1}^{P}, 0.3}^{2} \widehat{\mathrm{c}}_{i}(A)$, and the same applies for $B$ and $C$

(2L) $\underline{R}_{\sum_{i=1}^{P}, 0.3}^{2} \widehat{\mathrm{c}}_{i}(A) \subseteq \underline{R}^{P, 0.3} \hat{\sum}_{i=1}^{2} \widehat{\mathrm{c}}_{i}(B)$

(2H) $\bar{R}_{\sum_{i=1}^{P}, \widehat{\mathrm{C}}_{i}}^{2.3}(A) \subseteq \bar{R}_{\sum_{i=1}^{P, 0.3} \widehat{\mathrm{c}}_{i}}^{(B)}$

$$
\underline{R}_{\sum_{i=1}^{P, 0} \widehat{\mathrm{C}}_{i}}^{2,0.3}(A \cap C)=\underline{R}_{\sum_{i=1}^{P, 0.3} \widehat{\mathrm{c}}_{i}}^{2}(A) \cap \underline{R}_{\sum_{i=1}^{P, \alpha} \widehat{\mathrm{c}}_{i}}^{(C)}(C)
$$

$\underline{R}^{P, 0.3} \sum_{i=1}^{2} \widehat{\mathrm{C}}_{i}(A \cup C) \supseteq \underline{R}_{\sum_{i=1}^{2} \hat{\mathrm{C}}_{i}}^{P, 0.3}(A) \cup \underline{R}_{\sum_{i=1}^{P, \alpha} \widehat{\mathrm{c}}_{i}}^{P(C)}$

$(3 \mathrm{H}) \bar{R}_{\sum_{i=1}^{P}, 0.3}^{2} \widehat{\mathrm{c}}_{i}(A \cap C) \subseteq \bar{R}_{\sum_{i=1}^{2}, 0.3}^{2} \widehat{\mathrm{c}}_{i}(A) \cap \bar{R}_{\sum_{i=1}^{P, \alpha} \widehat{\mathrm{c}}_{i}}(C), \bar{R}_{\sum_{i=1}^{2}}^{P, 0.3} \widehat{\mathrm{c}}_{i}$ $(A \cup C)=\bar{R}_{\sum_{i=1}^{2}, \widehat{\mathrm{c}}_{i}}^{P, 3}(A) \cup \bar{R}_{\sum_{i=1}^{2}, \alpha}^{2, \alpha} \widehat{\mathrm{c}}_{i}(C)$

(4LH) Since $\frac{R}{R^{P, 0.3}} \hat{\sum}_{i=1}^{n} \hat{\mathrm{c}}_{i}\left(A^{\prime}\right)=\left(0.2 / x_{1}\right)+\left(0.2 / x_{2}\right)+(0.2 /$ $\left.x_{3}\right)+\left(0.5 / x_{4}\right)+\left(0.2 / x_{5}\right)$ and $\bar{R}_{\sum_{i=1}^{n} \widehat{\mathrm{C}}_{i}}^{P, 0.3}\left(A^{\prime}\right)=\left(0.6 / x_{1}\right)+$ $\left(0.8 / x_{2}\right)+\left(0.6 / x_{3}\right)+\left(0.8 / x_{4}\right)+\left(0.8 / x_{5}\right), \quad$ then

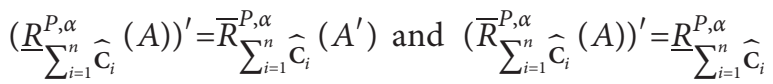
$\left(A^{\prime}\right)$.

(5LH) Since $\underline{R}^{P, 0.5} \sum_{i=1}^{2} \widehat{\mathrm{c}}_{i}\left(A^{\prime}\right)=\left(0.4 / x_{1}\right)+\left(0.4 / x_{2}\right)+\left(0.6 / x_{3}\right)$ $+\left(0.2 / x_{4}\right)+\left(0.5 / x_{5}\right)$ and $\bar{R}_{\sum_{i=1}^{P}, 0.5}^{2} \widehat{\mathrm{c}}_{i}(A)=\left(0.8 / x_{1}\right)+\left(0.8 / x_{2}\right)$ $+\left(0.6 / x_{3}\right)+\left(0.2 / x_{4}\right)+\left(0.5 / x_{5}\right)$, then $\underline{R}_{\sum_{i=1}^{P}, 0.3}^{{ }_{0}} \widehat{\mathrm{c}}_{i}(A) \subseteq \underline{R}^{P, 0.5} \sum_{i=1}^{n} \widehat{\mathrm{c}}_{i}$ (A) and $\bar{R}_{\sum_{i=1}^{n}, 0.3}^{n} \widehat{\mathbf{c}}_{i}(A) \supseteq \bar{R}_{\sum_{i=1}^{n}, 0.5}^{n} \widehat{\mathbf{c}}_{i}(A)$

3.3. Relationships between CMFRSs. In this section, we give some relationships between CMFRSs. Firstly, the relationship between covering-based optimistic and pessimistic multigranulation fuzzy rough sets is proposed.

Theorem 1. Let $\widetilde{\Gamma}=\left\{\widehat{\mathbf{C}}_{1}, \ldots, \widehat{\mathbf{C}}_{n}\right\}$ be an $n$-fuzzy $\beta$-coverings of $U$. Then, for any $A \in F(U)$,

$S \underline{R}^{P} \sum_{i=1}^{n} \widehat{\mathrm{c}}_{i}(A) \subseteq S \underline{R}_{\sum_{i=1}^{n} \widehat{\mathrm{c}}_{i}}^{O}(A) \subseteq A \subseteq S \bar{R}_{\sum_{i=1}^{n} \widehat{\mathrm{c}}_{i}}^{O}(A) \subseteq S \bar{R}_{\sum_{i=1}^{n} \widehat{\mathrm{c}}_{i}}^{P}(A)$.

Proof. By Definitions 5 and 7 and Proposition 1, it is immediate.

Example 10 (continued from Example 1). In Examples 2 and 6, we know that $A=\left(0.8 / x_{1}\right)+\left(0.4 / x_{2}\right)+\left(0.6 / x_{3}\right)+(0.2 /$ $\left.x_{4}\right)+\left(0.5 / x_{5}\right), \quad S \underline{R}_{\sum_{i=1}^{2} \widehat{\mathrm{c}}_{i}}^{O}(A)=\left(0.6667 / x_{1}\right)+\left(0.4 / \quad x_{2}\right)$ $+\left(0.6 / x_{3}\right)+\left(0.2 / x_{4}\right)+\left(0.5 / x_{5}\right), S \bar{R}_{\sum_{i=1}^{2} \widehat{\mathrm{C}}_{i}}^{O}(A)=\left(0.8 / x_{1}\right)+$ $\left(0.4 / x_{2}\right)+\left(0.6 / x_{3}\right)+\left(0.333 / x_{4}\right)+\left(0.5 / x_{5}\right), \quad S \underline{R}_{\sum_{i=1}^{2} \widehat{\mathrm{c}}_{i}}^{P}(A)$ $=\left(0.4 / x_{1}\right)+\left(0.4 / x_{2}\right)+\left(0.6 / x_{3}\right)+\left(0.2 / x_{4}\right)+\left(0.5 / x_{5}\right)$, and $S \bar{R}_{\sum_{i=1}^{2} \widehat{\mathrm{C}}_{i}}(A)=\left(0.8 / x_{1}\right)+\left(0.8 / x_{2}\right)+\left(0.6 / x_{3}\right)+\left(0.5 / x_{4}\right)+$ $\left(0.5 / x_{5}\right)$.

Hence, $S \underline{R}_{\sum_{i=1}^{2} \widehat{\mathrm{c}}_{i}}^{P}(A) \subseteq S \underline{R}_{\sum_{i=1}^{O} \widehat{\mathrm{c}}_{i}}^{O}(A) \subseteq A \subseteq S \bar{R}_{\sum_{i=1}^{2} \widehat{\mathrm{c}}_{i}}(A) \subseteq S \bar{R}_{\sum_{i=1}^{P} \widehat{\mathrm{c}}_{i}}(A)$.

Then, the relationship between $\alpha$-covering-based optimistic and pessimistic multigranulation fuzzy rough sets is given.

Theorem 2. Let $\widetilde{\Gamma}=\left\{\widehat{\mathbf{C}}_{1}, \ldots, \widehat{\mathbf{C}}_{n}\right\}$ be an $n$-fuzzy $\beta$-coverings of $U$. Then, for any $A \in F(U)$,

$$
S \underline{R}_{\sum_{i=1}^{n, \alpha} \widehat{\mathrm{c}}_{i}}^{P}(A) \subseteq S \underline{R}_{\sum_{i=1}^{n, \alpha} \widehat{\mathrm{c}}_{i}}^{O, A} \subseteq A \subseteq S \bar{R}_{\sum_{i=1}^{O, \alpha} \widehat{\mathrm{c}}_{i}}(A) \subseteq S \bar{R}_{\sum_{i=1}^{P, \alpha} \widehat{\mathrm{c}}_{i}}(A) .
$$

Proof. By Definitions 6 and 8 and Proposition 3, it is immediate.

Example 11 (continued from Example 1). In Examples 4 and 8, we know that $\underline{R}_{\sum_{i=1}^{O} \hat{\mathrm{c}}_{i}}^{O, 0.3}(A)=\left(0.5 / x_{1}\right)+\left(0.4 / x_{2}\right)+(0.6 /$ $\left.x_{3}\right)+\left(0.2 / x_{4}\right)+\left(0.4 / x_{5}\right), \quad \bar{R}_{\sum_{i=1}^{O}, 0.3}^{2} \widehat{\mathrm{c}}_{i}(A)=\left(0.8 / x_{1}\right)+\left(0.5 / x_{2}\right)$ $+\left(0.8 / x_{3}\right)+\left(0.4 / x_{4}\right)+\left(0.5 / x_{5}\right), \quad \underline{R}^{P, 0.3} \sum_{i=1}^{2} \widehat{\mathrm{c}}_{i}(A)=\left(0.4 / x_{1}\right)+$ $\left(0.2 / x_{2}\right)+\left(0.4 / x_{3}\right)+\left(0.2 / x_{4}\right)+\left(0.2 / x_{5}\right)$, and $\bar{R}_{\sum_{i=1}^{2}{ }^{P} \widehat{\mathrm{c}}_{i}}(A)=$ $\left(0.8 / x_{1}\right)+\left(0.8 / x_{2}\right)+\left(0.8 / x_{3}\right)+\left(0.5 / x_{4}\right)+\left(0.8 / x_{5}\right)$. Hence,

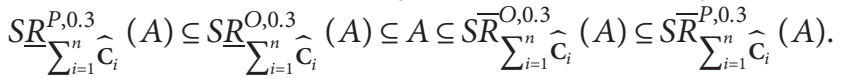

\section{Matrix Approaches for CMFRSs}

In this section, we mainly use matrix approaches to calculate approximation operators of CMFRS models presented in Section 3. They are the OMNFLAO $S \underline{R}_{\sum_{i=1}^{n}}^{O} \widehat{\mathrm{C}}_{i}(A)$ and the OMNFUAO $S \bar{R}_{\sum_{i=1}^{O} \widehat{\mathrm{C}}_{i}}^{O}(A) ; \alpha$-OMNFLAO $\underline{R}_{\sum_{i=1}^{n} \widehat{\mathrm{c}}_{i}, \alpha}^{\mathrm{c}_{i}}(A)$ and the $\alpha$-OMNFUAO $\bar{R}_{\sum_{i=1}^{n}, \alpha}^{0} \widehat{\mathrm{c}}_{i}(A)$; the PMNFLAO $S \underline{R}^{P} \sum_{i=1}^{n} \widehat{\mathrm{c}}_{i}(A)$ and PMNFUAO $S \bar{R}_{\sum_{i=1}^{n} \widehat{\mathrm{c}}_{i}}(A)$; and the $\alpha$-PMNFLAO $\underline{R}_{\sum_{i=1}^{P, \alpha} \widehat{\mathrm{c}}_{i}}^{P}(A)$ and the $\alpha$-PMNFUAO $\bar{R}_{\sum^{n} \widehat{\mathbf{C}}_{i}, \alpha}(A)$.

In [24], several matrices and matrix operations are presented in fuzzy covering-based rough sets. Hence, we consider the statement that $n=1$ firstly; that is, $\widehat{\mathbf{C}}_{1}=\cdots=\widehat{\mathbf{C}}_{n}=\widehat{\mathbf{C}}$.

Definition 9 (see [24]). Let $\widehat{\mathbf{C}}=\left\{C_{1}, C_{2}, \ldots, C_{m}\right\}$ be a fuzzy $\beta$-covering of $U=\left\{x_{1}, \ldots, x_{n}\right\}$. We call $M_{\widehat{\mathrm{C}}}=\left(m_{i j}\right)_{n \times m}=$ $\left(C_{j}\left(x_{i}\right)\right)_{n \times m}$ a matrix representation of $\widehat{\mathrm{C}}$ and call the 
Boolean matrix $M_{\widehat{\mathrm{C}}}^{\beta}=\left(t_{i j}\right)_{n \times m}$ a $\beta$-matrix representation of $M_{\widehat{C}}$, where

$$
\begin{aligned}
t_{i j} & = \begin{cases}1, & m_{i j} \geq \beta, \\
0, & m_{i j}<\beta,\end{cases} \\
\text { i.e., } t_{i j} & = \begin{cases}1, & C_{j}\left(x_{i}\right) \geq \beta, \\
0, & C_{j}\left(x_{i}\right)<\beta .\end{cases}
\end{aligned}
$$

Example 12 (continued from Example 1). First, we have

$$
\begin{aligned}
& M_{\widehat{C}_{1}}=\left(\begin{array}{llll}
0.8 & 0.4 & 0.3 & 0.7 \\
0.3 & 0.6 & 0.5 & 0.4 \\
0.7 & 0.2 & 0.6 & 0.8 \\
0.8 & 0.7 & 0.7 & 0.5 \\
0.8 & 0.9 & 0.4 & 0.6
\end{array}\right), \\
& M_{\widehat{C}_{2}}=\left(\begin{array}{llll}
0.4 & 0.7 & 0.7 & 0.3 \\
0.3 & 0.8 & 0.6 & 0.5 \\
0.8 & 0.6 & 0.7 & 0.4 \\
0.6 & 0.3 & 0.3 & 0.7 \\
0.7 & 0.4 & 0.6 & 0.8
\end{array}\right) .
\end{aligned}
$$

Then,

$$
\begin{aligned}
M_{\widehat{\mathrm{C}}_{1}}^{0.6} & =\left(\begin{array}{llll}
1 & 0 & 0 & 1 \\
0 & 1 & 0 & 0 \\
1 & 0 & 1 & 1 \\
1 & 1 & 1 & 0 \\
1 & 1 & 0 & 1
\end{array}\right), \\
M_{\widehat{\mathrm{C}}_{2}}^{0.6} & =\left(\begin{array}{llll}
0 & 1 & 1 & 0 \\
0 & 1 & 1 & 0 \\
1 & 1 & 1 & 0 \\
1 & 0 & 0 & 1 \\
1 & 0 & 1 & 1
\end{array}\right) .
\end{aligned}
$$

Let $A=\left(a_{i j}\right)_{m \times l}$ and $B=\left(b_{j k}\right)_{l \times n}$ be two matrices. Then, $C=A * B=\left(c_{i k}\right)_{m \times n}$ and $D=A \cdot B=\left(d_{i k}\right)_{m \times n}$ are defined as follows: $c_{i k}=\wedge_{j=1}^{l}\left[\left(1-a_{i j}\right) \vee b_{j k}\right]$ and $d_{i k}=\vee_{j=1}^{l}\left[a_{i j} \wedge b_{j k}\right]$, where $i=1,2, \ldots, m$ and $k=1,2, \ldots, n . A^{T}$ denotes the transpose of $A$, and $1_{m \times n}$ is a matrix with all entries being 1 . Then, the matrix representation of $\widetilde{\mathcal{N}}_{\widehat{\mathrm{C}}(x)}^{\beta}(x \in U)$ is presented in Lemma 1.

Lemma 1 (see [24]). Let $\widehat{\mathbf{C}}=\left\{C_{1}, C_{2}, \ldots, C_{m}\right\}$ be a fuzzy $\beta$-covering of $U=\left\{x_{1}, \ldots, x_{n}\right\}$. Then, $M_{\widehat{\mathbf{C}}}^{\beta} * M_{\widehat{\mathrm{C}}}^{T}=\left(\tilde{\mathcal{N}}_{\widehat{\mathbf{C}}\left(x_{i}\right)}^{\beta}\right.$ $\left.\left(x_{j}\right)\right)_{n \times n}$.

Example 13 (continued from Example 1). Based on Example 12 , we have

$$
\begin{aligned}
& M_{\widehat{\mathrm{C}}_{1}}^{0.6} * M_{\widehat{\mathrm{C}}_{1}}^{T}=\left(\begin{array}{cccc}
1 & 0 & 0 & 1 \\
0 & 1 & 0 & 0 \\
1 & 0 & 1 & 1 \\
1 & 1 & 1 & 0 \\
1 & 1 & 0 & 1
\end{array}\right) *\left(\begin{array}{cccc}
0.8 & 0.4 & 0.3 & 0.7 \\
0.3 & 0.6 & 0.5 & 0.4 \\
0.7 & 0.2 & 0.6 & 0.8 \\
0.8 & 0.7 & 0.7 & 0.5 \\
0.8 & 0.9 & 0.4 & 0.6
\end{array}\right)^{T} \\
& =\left(\begin{array}{ccccc}
0.7 & 0.3 & 0.7 & 0.5 & 0.6 \\
0.4 & 0.6 & 0.2 & 0.7 & 0.9 \\
0.3 & 0.3 & 0.6 & 0.5 & 0.4 \\
0.3 & 0.3 & 0.2 & 0.7 & 0.4 \\
0.4 & 0.3 & 0.2 & 0.5 & 0.6
\end{array}\right)=\left(\widetilde{\mathcal{N}}_{\widetilde{\mathrm{C}}_{1}\left(x_{i}\right)}^{0.6}\left(x_{j}\right)\right)_{5 \times 5}, \\
& M_{\widehat{\mathrm{C}}_{2}}^{0.6} * M_{\widehat{\mathrm{C}}_{2}}^{T}=\left(\begin{array}{cccc}
0 & 1 & 1 & 0 \\
0 & 1 & 1 & 0 \\
1 & 1 & 1 & 0 \\
1 & 0 & 0 & 1 \\
1 & 0 & 1 & 1
\end{array}\right) *\left(\begin{array}{cccc}
0.4 & 0.7 & 0.7 & 0.3 \\
0.3 & 0.8 & 0.6 & 0.5 \\
0.8 & 0.6 & 0.7 & 0.4 \\
0.6 & 0.3 & 0.3 & 0.7 \\
0.7 & 0.4 & 0.6 & 0.8
\end{array}\right)^{T} \\
& =\left(\begin{array}{lllll}
0.7 & 0.6 & 0.6 & 0.3 & 0.4 \\
0.7 & 0.6 & 0.6 & 0.3 & 0.4 \\
0.4 & 0.3 & 0.6 & 0.3 & 0.4 \\
0.3 & 0.3 & 0.4 & 0.6 & 0.7 \\
0.3 & 0.3 & 0.4 & 0.3 & 0.6
\end{array}\right)=\left(\widetilde{\mathcal{N}}_{\widehat{\mathrm{C}}_{2}\left(x_{i}\right)}^{0.6}\left(x_{j}\right)\right)_{5 \times 5} \text {. }
\end{aligned}
$$


Inspired by Definition 2 and Lemma 1, the matrix representation of $\overline{\mathcal{N}}_{\widehat{\mathrm{C}}(x)}^{\beta}$ is presented as follows.

Proposition 5. Let $\widehat{\mathbf{C}}=\left\{C_{1}, C_{2}, \ldots, C_{m}\right\}$ be a fuzzy $\beta$-covering of $U=\left\{x_{1}, \ldots, x_{n}\right\}$. Then, $\left(M_{\widehat{\mathbf{C}}}^{\beta} * M_{\widehat{\mathrm{C}}}^{T}\right)^{\beta}=\left(\overline{\mathcal{N}}_{\widehat{\mathrm{C}}\left(x_{i}\right)}^{\beta}\right.$ $\left.\left(x_{j}\right)\right)_{n \times n}$, where $\overline{\mathcal{N}}_{\widehat{\mathbf{C}}\left(x_{i}\right)}^{\beta}\left(x_{j}\right)= \begin{cases}1 & x_{j} \in \overline{\mathscr{N}}_{\widehat{\mathrm{C}}\left(x_{i}\right)}^{\beta} . \\ 0 & \text { otherwise }\end{cases}$
Proof. By Lemma 1, $M_{\widehat{\mathrm{C}}}^{\beta} * M_{\widehat{\mathrm{C}}}^{T}=\left(\overline{\mathcal{N}}_{\widehat{\mathrm{C}}\left(x_{i}\right)}^{\beta}\left(x_{j}\right)\right)_{n \times n}$. Suppose that $\left(M_{\widehat{\mathrm{C}}}^{\beta} * M_{\widehat{\mathrm{C}}}^{T}\right)^{\beta}=\left(a_{i j}\right)_{n \times n}^{\mathrm{C}}$. Then,

$$
a_{i j}=\left\{\begin{array}{ll}
1 & \tilde{\mathcal{N}}_{\widehat{\mathrm{C}}\left(x_{i}\right)}^{\beta}\left(x_{j}\right) \geq \beta ; \\
0 & \text { otherwise }
\end{array}=\left\{\begin{array}{ll}
1 & x_{j} \in \overline{\mathcal{N}}_{\widehat{\mathrm{C}}\left(x_{i}\right)}^{\beta}=\tilde{\mathcal{N}}_{\widehat{\mathrm{C}}\left(x_{i}\right)}^{\beta} \\
0 & \text { otherwise }
\end{array}\left(x_{j}\right) .\right.\right.
$$

Example 14 (continued from Example 1). Based on Example 13 , we have

$$
\begin{aligned}
& \left(M_{\widehat{\mathrm{C}}_{1}}^{0.6} * M_{\widehat{\mathrm{C}}_{1}}^{T}\right)^{0.6}=\left(\begin{array}{ccccc}
0.7 & 0.3 & 0.7 & 0.5 & 0.6 \\
0.4 & 0.6 & 0.2 & 0.7 & 0.9 \\
0.3 & 0.3 & 0.6 & 0.5 & 0.4 \\
0.3 & 0.3 & 0.2 & 0.7 & 0.4 \\
0.4 & 0.3 & 0.2 & 0.5 & 0.6
\end{array}\right)=\left(\begin{array}{lllll}
1 & 0 & 1 & 0 & 1 \\
0 & 1 & 0 & 1 & 1 \\
0 & 0 & 1 & 0 & 0 \\
0 & 0 & 0 & 1 & 0 \\
0 & 0 & 0 & 0 & 1
\end{array}\right)=\left(\widehat{N}_{\widehat{\mathrm{C}}_{1}\left(x_{i}\right)}^{0.6}\left(x_{j}\right)\right)_{5 \times 5}, \\
& \left(M_{\widehat{\mathrm{C}}_{2}}^{0.6} * M_{\widehat{\mathrm{C}}_{2}}^{T}\right)^{0.6}=\left(\begin{array}{ccccc}
0.7 & 0.6 & 0.6 & 0.3 & 0.4 \\
0.7 & 0.6 & 0.6 & 0.3 & 0.4 \\
0.4 & 0.3 & 0.6 & 0.3 & 0.4 \\
0.3 & 0.3 & 0.4 & 0.6 & 0.7 \\
0.3 & 0.3 & 0.4 & 0.3 & 0.6
\end{array}\right)=\left(\begin{array}{ccccc}
1 & 1 & 1 & 0 & 0 \\
1 & 1 & 1 & 0 & 0 \\
0 & 0 & 1 & 0 & 0 \\
0 & 0 & 0 & 1 & 1 \\
0 & 0 & 0 & 0 & 1
\end{array}\right)=\left(\overline{\mathcal{N}}_{\widehat{\mathrm{C}}_{2}\left(x_{i}\right)}^{0.6}\left(x_{j}\right)\right)_{5 \times 5} .
\end{aligned}
$$

In what follows, the definition of the matrix representation of all multigranulation fuzzy neighborhood measures is presented.

Definition 10. Let $\widehat{\mathbf{C}}=\left\{C_{1}, C_{2}, \ldots, C_{m}\right\}$ be a fuzzy $\beta$-covering of $U=\left\{x_{1}, \ldots, x_{n}\right\}$. We call $D_{\widehat{c}}^{\beta^{m}}=\left(d_{i j}\right)_{n \times n}$ the matrix representation of all multigranulation fuzzy neighborhood measures $D_{\widehat{\mathrm{C}}}^{\beta}\left(x_{i}, x_{j}\right) \quad(i, j=1,2, \ldots, n)$, where $d_{i j}=D_{\widehat{\mathrm{C}}}^{\beta}\left(x_{i}, x_{j}\right)$. $^{\mathrm{C}}$

Proposition 6 shows that the matrix representation of all multigranulation fuzzy neighborhood measures is symmetric.
Proposition 6. Let $\widehat{\mathbf{C}}=\left\{C_{1}, C_{2}, \ldots, C_{m}\right\}$ be a fuzzy $\beta$-covering of $U=\left\{x_{1}, \ldots, x_{n}\right\}$. Then, $D_{\widehat{\mathrm{C}}}^{\beta}$ is a symmetric matrix. Proof. By Definitions 4 and 10, it is immediate.

Let $A=\left(a_{i j}\right)_{m \times l}, B=\left(b_{j k}\right)_{l \times n}$, and $E=\left(e_{i j}\right)_{m \times l}$ be three matrices. Then, $C=A \nabla B=\left(c_{i k}\right)_{m \times n}, D=A \Delta B=\left(d_{i k}\right)_{m \times n}$, and $G=A \oslash E=\left(g_{j j}\right)_{m \times l}$ are defined as follows: $c_{i k}=\sum_{j=1}^{l}$ $\left[a_{i j} \wedge b_{j k}\right], \quad d_{i k}=\sum_{j=1}^{f}\left[a_{i j} \vee b_{j k}\right] \quad$ and $g_{i j}=a_{i j} / e_{i \dot{\beta}}$, where $i=1,2, \ldots, m$ and $k=1,2, \ldots, n$. Hence, all $D_{\widehat{c}}^{\beta}$ can be calculated by matrices.

Proposition 7. Let $\widehat{\mathbf{C}}=\left\{C_{1}, C_{2}, \ldots, C_{m}\right\}$ be a fuzzy $\beta$-covering of $U=\left\{x_{1}, \ldots, x_{n}\right\}$. Then,

$$
D_{\widehat{\mathrm{C}}}^{\beta}=\left(\left(M_{\widehat{\mathrm{C}}}^{\beta} * M_{\widehat{\mathrm{C}}}^{T}\right)^{\beta} \nabla\left(M_{\widehat{\mathrm{C}}}^{\beta} * M_{\widehat{\mathrm{C}}}^{T}\right)^{\beta, T}\right) \oslash\left(\left(M_{\widehat{\mathrm{C}}}^{\beta} * M_{\widehat{\mathrm{C}}}^{T}\right)^{\beta} \triangle\left(M_{\widehat{\mathrm{C}}}^{\beta} * M_{\widehat{\mathrm{C}}}^{T}\right)^{\beta, T}\right)
$$


where $\left(M_{\widehat{\mathrm{C}}}^{\beta} * M_{\widehat{\mathrm{C}}}^{T}\right)^{\beta, T}$ means $\left(\left(M_{\widehat{\mathrm{C}}}^{\beta} * M_{\widehat{\mathrm{C}}}^{T}\right)^{\beta}\right)^{T}$.

Proof. Suppose that $D_{\widehat{\mathrm{C}}}^{\beta}=\left(d_{i j}\right)_{n \times n},\left(\left(M_{\widehat{\mathrm{C}}}^{\beta} * M_{\widehat{\mathrm{C}}}^{T}\right)^{\beta} \nabla\left(M_{\widehat{\mathrm{C}}}^{\beta} *\right.\right.$ $\left.\left.M_{\widehat{\mathrm{C}}}^{T}\right)^{\beta, T}\right)=\left(c_{i j}\right)_{n \times n}$ and $\left(\left(M_{\widehat{\mathrm{C}}}^{\beta} * M_{\widehat{\mathrm{C}}}^{T}\right)^{\beta} \triangle\left(M_{\widehat{\mathrm{C}}}^{\beta} * M_{\widehat{\mathrm{C}}}^{T}\right)^{\beta, T}\right)=$ $\left(b_{i j}\right)_{n \times n}$. By Proposition 5, $\left(M_{\widehat{\mathrm{C}}}^{\beta} * M_{\widehat{\mathrm{C}}}^{T}\right)^{\beta}=\left(\overline{\mathcal{N}}_{\widehat{\mathrm{C}}\left(x_{i}\right)}^{\beta}\left(x_{j}\right)\right)_{n \times n}$.
Hence, $\quad c_{i j}=\overline{\mathcal{N}}_{\hat{\mathbf{C}}\left(x_{i}\right)}^{\beta} \cap \overline{\mathcal{N}}_{\widehat{\mathbf{C}}\left(x_{j}\right)}^{\beta}$ and $b_{i j}=\overline{\mathcal{N}}_{\hat{\mathbf{C}}\left(x_{i}\right)}^{\beta} \cup \overline{\mathcal{N}}_{\widehat{\mathbf{C}}_{i}\left(x_{j}\right)}^{\beta}$. Therefore, $d_{i j}=c_{i j} / b_{i j}=D_{\widehat{\mathrm{C}}}^{\beta}\left(x_{i}, x_{j}\right)$.

Example 15 (continued from Example 1). Based on Example 14 , we have $D_{\overline{\mathrm{C}}_{1}}^{0.6}=\left(\left(\begin{array}{ccccc}1 & 0 & 1 & 0 & 1 \\ 0 & 1 & 0 & 1 & 1 \\ 0 & 0 & 1 & 0 & 0 \\ 0 & 0 & 0 & 1 & 0 \\ 0 & 0 & 0 & 0 & 1\end{array}\right) \nabla\left(\begin{array}{ccccc}1 & 0 & 1 & 0 & 1 \\ 0 & 1 & 0 & 1 & 1 \\ 0 & 0 & 1 & 0 & 0 \\ 0 & 0 & 0 & 1 & 0 \\ 0 & 0 & 0 & 0 & 1\end{array}\right)^{T}\left(\left(\begin{array}{lllll}1 & 0 & 1 & 0 & 1 \\ 0 & 1 & 0 & 1 & 1 \\ 0 & 0 & 1 & 0 & 0 \\ 0 & 0 & 0 & 1 & 0 \\ 0 & 0 & 0 & 0 & 1\end{array}\right)^{T}\left(\begin{array}{lllll}1 & 0 & 1 & 0 & 1 \\ 0 & 1 & 0 & 1 & 1 \\ 0 & 0 & 1 & 0 & 0 \\ 0 & 0 & 0 & 1 & 0 \\ 0 & 0 & 0 & 0 & 1\end{array}\right)^{T}\right.\right.$

$$
\begin{aligned}
& =\left(\begin{array}{lllll}
3 & 1 & 1 & 0 & 1 \\
1 & 3 & 0 & 1 & 1 \\
1 & 0 & 1 & 0 & 0 \\
0 & 1 & 0 & 1 & 0 \\
1 & 1 & 0 & 0 & 1
\end{array}\right)\left(\begin{array}{lllll}
3 & 5 & 3 & 4 & 3 \\
5 & 3 & 4 & 3 & 3 \\
3 & 4 & 1 & 2 & 2 \\
4 & 3 & 2 & 1 & 2 \\
3 & 3 & 2 & 2 & 1
\end{array}\right)=\left(\begin{array}{ccccc}
1 & \frac{1}{5} & \frac{1}{3} & 0 & \frac{1}{3} \\
\frac{1}{5} & 1 & 0 & \frac{1}{3} & \frac{1}{3} \\
\frac{1}{3} & 0 & 1 & 0 & 0 \\
0 & \frac{1}{3} & 0 & 1 & 0 \\
\frac{1}{3} & \frac{1}{3} & 0 & 0 & 1
\end{array}\right), \\
& D_{\overrightarrow{\mathrm{C}}_{2}}^{0.6}=\left(\begin{array}{ccccc}
3 & 3 & 1 & 0 & 0 \\
3 & 3 & 1 & 0 & 0 \\
1 & 1 & 1 & 0 & 0 \\
0 & 0 & 0 & 2 & 1 \\
0 & 0 & 0 & 1 & 1
\end{array}\right)\left(\begin{array}{lllll}
3 & 3 & 3 & 5 & 4 \\
3 & 3 & 3 & 5 & 4 \\
3 & 3 & 1 & 3 & 2 \\
5 & 5 & 3 & 2 & 2 \\
4 & 4 & 2 & 2 & 1
\end{array}\right)=\left(\begin{array}{lllll}
1 & 1 & \frac{1}{3} & 0 & 0 \\
\frac{1}{3} & \frac{1}{3} & 1 & 0 & 0 \\
1 & 1 & \frac{1}{3} & 0 & 0 \\
0 & 0 & 0 & 1 & \frac{1}{2} \\
0 & 0 & 0 & \frac{1}{2} & 1
\end{array}\right) .
\end{aligned}
$$


Then, we also investigate the matrix representations of the OMNFLAO $S \underline{R}_{\sum_{i=1}^{O}}^{O} \widehat{\mathrm{C}}_{i}(A)$ and the OMNFUAO $S \bar{R}_{\sum_{i=1}^{n} \widehat{\mathrm{C}}_{i}}^{O}(A)$. Suppose that $n=1$; that is, $S \underline{R}_{\widehat{\mathrm{C}}}^{O}(A)$ and the OMNFUAO $S \bar{R}_{\widehat{\mathrm{C}}}^{O}(A)$ should be considered firstly.

Proposition 8. Let $\widehat{\mathbf{C}}=\left\{C_{1}, C_{2}, \ldots, C_{m}\right\}$ be a fuzzy $\beta$-covering of $U=\left\{x_{1}, \ldots, x_{n}\right\}$. Then, for any $A \in F(U)$,

$$
\begin{aligned}
& S \underline{R}_{\widehat{\mathrm{C}}}^{O}(A)=D_{\widehat{\mathrm{C}}}^{\beta} * A, \\
& S \bar{R}_{\widehat{\mathrm{C}}}^{O}(A)=D_{\widehat{\mathrm{C}}}^{\beta} \cdot A .
\end{aligned}
$$

Proof. By Definition 5, it is immediate.

Example 16 (continued from Example 1). Based on Example 15 , we have

$$
\begin{aligned}
& S \underline{R}_{\widehat{\mathrm{C}}_{1}}^{O}(A)=D_{\widehat{\mathrm{C}}_{1}}^{0.6} * A=\left(\begin{array}{ccccc}
1 & \frac{1}{5} & \frac{1}{3} & 0 & \frac{1}{3} \\
\frac{1}{5} & 1 & 0 & \frac{1}{3} & \frac{1}{3} \\
\frac{1}{3} & 0 & 1 & 0 & 0 \\
0 & \frac{1}{3} & 0 & 1 & 0 \\
\frac{1}{3} & \frac{1}{3} & 0 & 0 & 1
\end{array}\right) *\left(\begin{array}{c}
0.8 \\
0.4 \\
0.6 \\
0.2 \\
0.5
\end{array}\right)=\left(\begin{array}{c}
0.6667 \\
0.4 \\
0.6 \\
0.2 \\
0.5
\end{array}\right) \text {, } \\
& S \bar{R}_{\widehat{\mathrm{C}}_{1}}^{O}(A)=D_{\widetilde{\mathrm{C}}_{1}}^{0.6} \cdot A=\left(\begin{array}{ccccc}
1 & \frac{1}{5} & \frac{1}{3} & 0 & \frac{1}{3} \\
\frac{1}{5} & 1 & 0 & \frac{1}{3} & \frac{1}{3} \\
\frac{1}{3} & 0 & 1 & 0 & 0 \\
0 & \frac{1}{3} & 0 & 1 & 0 \\
\frac{1}{3} & \frac{1}{3} & 0 & 0 & 1
\end{array}\right) \cdot\left(\begin{array}{l}
0.8 \\
0.4 \\
0.6 \\
0.2 \\
0.5
\end{array}\right)=\left(\begin{array}{c}
0.8 \\
0.4 \\
0.6 \\
0.333 \\
0.5
\end{array}\right),
\end{aligned}
$$

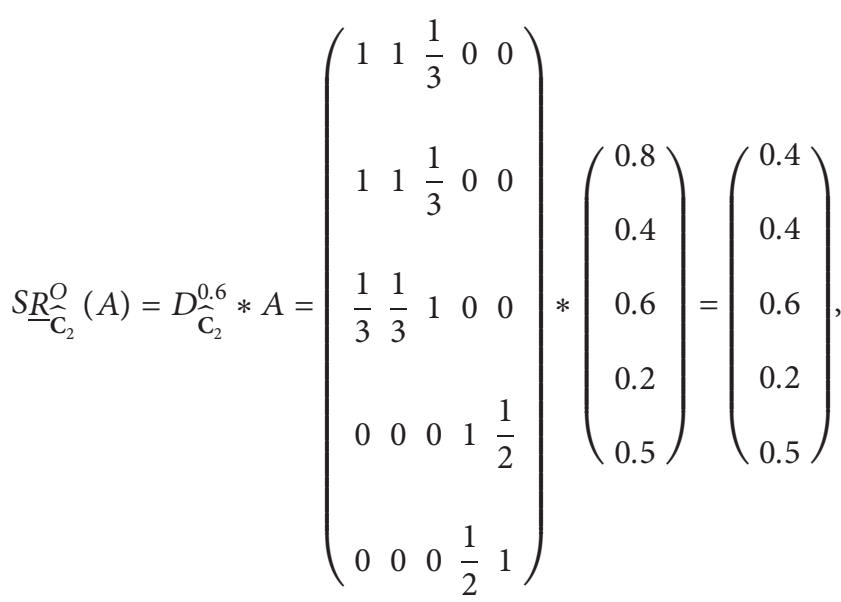




$$
S \bar{R}_{\widehat{\mathrm{C}}_{2}}^{O}(A)=D_{\widehat{\mathrm{C}}_{2}}^{0.6} \cdot A=\left(\begin{array}{ccccc}
1 & 1 & \frac{1}{3} & 0 & 0 \\
1 & 1 & \frac{1}{3} & 0 & 0 \\
\frac{1}{3} & \frac{1}{3} & 1 & 0 & 0 \\
0 & 0 & 0 & 1 & \frac{1}{2} \\
0 & 0 & 0 & \frac{1}{2} & 1
\end{array}\right) \cdot\left(\begin{array}{c}
0.8 \\
0.4 \\
0.6 \\
0.2 \\
0.5
\end{array}\right)=\left(\begin{array}{c}
0.8 \\
0.8 \\
0.6 \\
0.5 \\
0.5
\end{array}\right) .
$$

Inspired by Definition 5 and Proposition 8, we give the matrix representations of the OMNFLAO $S \underline{R}_{\sum_{i=1}^{n}}^{O} \widehat{\mathrm{C}}_{i}(A)$ and the OMNFUAO $S \bar{R}_{\sum_{i=1}^{n} \widehat{\mathrm{C}}_{i}}(A)$.

Theorem 3. Let $\widetilde{\Gamma}=\left\{\widehat{\mathbf{C}}_{1}, \ldots, \widehat{\mathbf{C}}_{n}\right\}$ be an $n$-fuzzy $\beta$-coverings of $U$. Then, for any $A \in F(U)$,

$$
\begin{aligned}
& S \underline{R}_{\sum_{i=1}^{n} \widehat{\mathbf{C}}_{i}}^{O}(A)=\bigvee_{i=1}^{n}\left(D_{\widehat{\mathbf{C}}_{i}}^{\beta} * A\right), \\
& S \bar{R}_{\sum_{i=1}^{n} \widehat{\mathbf{C}}_{i}}^{O}(A)=\bigwedge_{i=1}^{n}\left(D_{\widehat{\mathbf{C}}_{i}}^{\beta} \cdot A\right) .
\end{aligned}
$$

Proof. By Definition 5 and Proposition 8, it is immediate.

Example 17 (continued from Example 1). Based on Example 16 , we have

$$
\begin{aligned}
S \underline{R}_{\sum_{i=1}^{2} \widehat{\mathrm{C}}_{i}}^{O}(A) & \bigvee_{i=1}^{2}\left(D_{\widehat{\mathrm{C}}_{i}}^{0.6} * A\right)=\left(\begin{array}{c}
0.6667 \\
0.4 \\
0.6 \\
0.2 \\
0.5
\end{array}\right) \bigvee\left(\begin{array}{l}
0.4 \\
0.4 \\
0.6 \\
0.2 \\
0.5
\end{array}\right)=\left(\begin{array}{c}
0.6667 \\
0.8 \\
0.4 \\
0.6 \\
0.2 \\
0.5
\end{array}\right) \\
S \bar{R}_{\sum_{i=1}^{2} \widehat{\mathrm{C}}_{i}}^{O}(A) & =\bigwedge_{i=1}^{2}\left(D_{\widehat{\mathrm{C}}_{i}}^{0.6} \cdot A\right)=\left(\begin{array}{c}
0.8 \\
0.6 \\
0.333 \\
0.5
\end{array}\right) \wedge\left(\begin{array}{c}
0.8 \\
0.6 \\
0.4 \\
0.6 \\
0.5
\end{array}\right)=\left(\begin{array}{c}
0.333 \\
0.5
\end{array}\right)
\end{aligned}
$$

Inspired by Definition 7 and Proposition 8, we give the matrix representations of the OMNFLAO $S \underline{R}^{P} \sum_{i=1}^{n} \widehat{\mathbf{C}}_{i}(A)$ and the OMNFUAO $S \bar{R}_{\sum_{i=1}^{n} \widehat{\mathrm{C}}_{i}}(A)$.

Theorem 4. Let $\widetilde{\Gamma}=\left\{\widehat{\mathbf{C}}_{1}, \ldots, \widehat{\mathbf{C}}_{n}\right\}$ be an $n$-fuzzy $\beta$-coverings of $U$. Then, for any $A \in F(U)$,

$$
\begin{aligned}
& S \underline{R}_{\sum_{i=1}^{n} \widehat{\mathrm{C}}_{i}}^{P}(A)=\bigwedge_{i=1}^{n}\left(D_{\widehat{\mathrm{C}}_{i}}^{\beta} * A\right), \\
& S \bar{R}_{\sum_{i=1}^{n} \widehat{\mathrm{C}}_{i}}^{P}(A)=\bigvee_{i=1}^{n}\left(D_{\widehat{\mathrm{C}}_{i}}^{\beta} \cdot A\right) .
\end{aligned}
$$

Proof. By Definition 7 and Proposition 8, it is immediate. 
Example 18 (continued from Example 1). Based on Example 16 , we have

$$
\begin{aligned}
S \underline{R}_{\sum_{i=1}^{2} \widehat{\mathrm{C}}_{i}}^{P}(A)=\bigwedge_{i=1}^{2}\left(D_{\widehat{\mathrm{C}}_{i}}^{0.6} * A\right)= & \left(\begin{array}{c}
0.6667 \\
0.4 \\
0.6 \\
0.2 \\
0.5
\end{array}\right) \wedge\left(\begin{array}{c}
0.4 \\
0.4 \\
0.6 \\
0.2 \\
0.5
\end{array}\right)=\left(\begin{array}{c}
0.4 \\
0.4 \\
0.6 \\
0.2 \\
0.5
\end{array}\right), \\
S \bar{R}_{\sum_{i=1}^{2} \widehat{\mathrm{C}}_{i}}^{P}(A)=\bigvee_{i=1}^{2}\left(D_{\widehat{\mathrm{C}}_{i}}^{0.6} \cdot A\right) & =\left(\begin{array}{c}
0.8 \\
0.4 \\
0.6 \\
0.333 \\
0.5
\end{array}\right) \bigvee\left(\begin{array}{l}
0.8 \\
0.8 \\
0.6 \\
0.5 \\
0.5
\end{array}\right)=\left(\begin{array}{c}
0.6 \\
0.5 \\
0.5
\end{array}\right) .
\end{aligned}
$$

Let $A=\left(a_{i j}\right)_{m \times l}$ and $B=\left(b_{j k}\right)_{l \times n}$ be two matrices. Then, $H=A \odot B=\left(h_{i k}\right)_{m \times n}$ and $T=A \circ B=\left(t_{i k}\right)_{m \times n}$ are defined as follows: $h_{i k}=\wedge_{j=1}^{l}\left[a_{i j} \wedge b_{j k}\right]$ and $t_{i k}=\vee_{j=1}^{l}\left[a_{i j} \vee b_{j k}\right]$, where $i=1,2, \ldots, m$ and $k=1,2, \ldots, n$. If $A$ is a Boolean matrix and $B=\left(b_{j}\right)_{l \times 1}$, then we define $C=A \triangleright B=\left(c_{i j}\right)_{m \times l}$ and $D=$ $A \triangleleft B=\left(d_{i j}\right)_{m \times l}$ as follows:

$$
\begin{aligned}
& c_{i j}= \begin{cases}b_{j}, & a_{i j}=1, \\
1, & a_{i j}=0,\end{cases} \\
& d_{i j}= \begin{cases}b_{j}, & a_{i j}=1, \\
0, & a_{i j}=0 .\end{cases}
\end{aligned}
$$

Finally, we investigate the matrix representations of the $\alpha$-OMNFLAO $\underline{R}_{\sum_{i=1}^{n, \alpha} \widehat{\mathrm{C}}_{i}}(A)$ and the $\alpha$-OMNFUAO $\bar{R}_{\sum_{i=1}^{O, \alpha} \widehat{\mathrm{C}}_{i}}$ (A); the $\alpha$-PMNFLAO $\underline{R}_{\sum_{i=1}^{P, \alpha} \widehat{C}_{i}}^{n}(A)$ and the $\alpha$-PMNFUAO $\bar{R}_{\sum_{i=1}^{n}{ }^{P, \alpha} \widehat{\mathrm{C}}_{i}}(A)$. Suppose that $n=1$; that is, the $\alpha$-OMNFLAO $\underline{R}_{\widehat{\mathrm{C}}}^{O, \alpha}(A)$ and the $\alpha$-OMNFUAO $\bar{R}_{\widehat{\mathrm{C}}}^{\mathrm{O}, \alpha}(A)$ (the $\alpha$-PMNFLAO $\underline{R}_{\widehat{\mathrm{C}}}^{P, \alpha}(A)$ and the $\alpha$-PMNFLAO $\left.\bar{R}_{\widehat{\mathrm{C}}}^{P, \alpha}(A)\right)$ should be considered firstly. Hence, for any $x \in U$,

$$
\begin{aligned}
& \underline{R}_{\widehat{\mathrm{C}}}^{O, \alpha}(A)(x)=\underline{R}_{\widehat{\mathrm{C}}}^{P, \alpha}(A)(x)=\bigwedge_{y \in U}\left\{A(y): D_{\widehat{\mathrm{C}}}^{\beta}(x, y) \geq \alpha\right\}, \\
& \bar{R}_{\widehat{\mathrm{C}}}^{O, \alpha}(A)(x)=\bar{R}_{\widehat{\mathrm{C}}}^{P, \alpha}(A)(x)=\bigvee_{y \in U}\left\{A(y): D_{\widehat{\mathrm{C}}}^{\beta}(x, y) \geq \alpha\right\} .
\end{aligned}
$$

Proposition 9. Let $\widehat{\mathbf{C}}$ be a fuzzy $\beta$-covering of $U=\left\{x_{1}, \ldots\right.$, $\left.x_{m}\right\}$ and $\alpha \in[0,1]$. Then, for any $A \in F(U)$,

$$
\begin{aligned}
& \underline{R}_{\widehat{\mathrm{C}}}^{O, \alpha}(A)=\left(D_{\widehat{\mathrm{C}}}^{\beta}\right)^{\alpha} \triangleright A \odot 1_{m \times 1}, \\
& \bar{R}_{\widehat{\mathrm{C}}}^{O, \alpha}(A)=\left(D_{\widehat{\mathrm{C}}}^{\beta}\right)^{\alpha} \triangleleft A \circ 0_{m \times 1},
\end{aligned}
$$

where $\triangleright$ and $\triangleleft$ have priority over $\odot$ and $\circ$, respectively.

Proof. Suppose that $\underline{R}_{\widehat{\mathrm{C}}}^{O, \alpha}(A)=\left(a_{i}\right)_{m \times 1},\left(D_{\widehat{\mathrm{C}}}^{\beta}\right)^{\alpha} \triangleright A=\left(c_{i j}\right)_{m \times m}$ and $\left(D_{\widehat{\mathrm{C}}}^{\beta}\right)^{\alpha}=\left(b_{i j}\right)_{m \times m}, \quad$ where $b_{i j}=\left\{\begin{array}{ll}1, & D_{\widehat{\mathrm{C}}}^{\beta}\left(x_{i}, x_{j}\right)=1 \\ 0, & \text { otherwise }\end{array}\right.$. Hence, $\quad c_{i j}=\left\{\begin{array}{ll}A\left(x_{j}\right), & b_{i j}=1 \\ 1, & b_{i j}=0\end{array}=\left\{\begin{array}{ll}A\left(x_{j}\right), & D_{\widehat{C}}^{\beta}\left(x_{i}, x_{j}\right) \geq \alpha \\ 1, & \text { otherwise }\end{array}\right.\right.$. Therefore, $\quad \underline{R}_{\widehat{\mathrm{C}}}^{O, \alpha}(A)\left(x_{i}\right)=a_{i}=\wedge_{j=1}^{m}\left[c_{i j} \wedge 1\right]=\wedge_{j=1}^{m} c_{i j}=\wedge_{j=1}^{m}$ $\left\{A\left(x_{j}\right): D_{\widehat{\mathrm{C}}}^{\beta}\left(x_{i}, x_{j}\right) \geq \alpha\right\}$.

On the other hand, suppose $\bar{R}_{\widehat{\mathrm{C}}}^{O, \alpha}(A)=\left(a_{i}^{\prime}\right)_{m \times 1},\left(D_{\widehat{\mathrm{C}}}^{\beta}\right)^{\alpha} \triangleleft$ $A=\left(c_{i j}^{\prime}\right)_{m \times m} . \quad$ Hence, $\quad c_{i j}^{\prime}= \begin{cases}A\left(x_{j}\right), & b_{i j}{ }^{\mathrm{C}}=1 \\ 0, & b_{i j}=0\end{cases}$ $=\left\{\begin{array}{ll}A\left(x_{j}\right), & D_{\widehat{\mathrm{C}}}^{\beta}\left(x_{i}, x_{j}\right) \geq \alpha \\ 0, & \text { otherwise }\end{array}\right.$. Therefore, $\bar{R}_{\widehat{\mathrm{C}}}^{O, \alpha}(A)\left(x_{i}\right)=a_{i}^{\prime}=$ $\vee_{j=1}^{m}\left[c_{i j} \vee 0\right]=\vee_{j=1}^{m} c_{i j}=\vee_{j=1}^{m}\left\{A\left(x_{j}\right): D_{\widehat{\mathbf{C}}}^{\beta}\left(x_{i}, x_{j}\right) \geq \alpha\right\}$.

Inspired by Definition 6 and Proposition 9, we present the matrix representations of the $\alpha$-OMNFLAO $\underline{R}_{\sum_{i=1}^{O, \alpha} \widehat{\mathrm{C}}_{i}}(A)$ and the $\alpha$-OMNFUAO $\bar{R}_{\sum_{i=1}^{n} \widehat{C}_{i}}^{\hat{C}_{i}}(A)$ as follows.

Theorem 5. Let $\widetilde{\Gamma}=\left\{\widehat{\mathbf{C}}_{1}, \ldots, \widehat{\mathbf{C}}_{n}\right\}$ be an $n$-fuzzy $\beta$-coverings of $U=\left\{x_{1}, \ldots, x_{m}\right\}$ and $\alpha \in[0,1]$. Then, for any $A \in F(U)$,

$$
\begin{aligned}
& \underline{R}_{\sum_{i=1}^{n} \widehat{\mathbf{C}}_{i}}^{O, \alpha}(A)=\bigvee_{i=1}^{n}\left(\left(D_{\widehat{\mathrm{C}}_{i}}^{\beta}\right)^{\alpha} \triangleright A \odot 1_{m \times 1}\right), \\
& \bar{R}_{\sum_{i=1}^{n} \widehat{\mathrm{C}}_{i}}^{O, \alpha}(A)=\bigwedge_{i=1}^{n}\left(\left(D_{\widehat{\mathrm{C}}_{i}}^{\beta}\right)^{\alpha} \triangleleft A \circ 0_{m \times 1}\right),
\end{aligned}
$$

where $\triangleright$ and $\triangleleft$ are first than $\odot$ and $\circ$, respectively.

Proof. By Definition 6 and Proposition 9, it is immediate.

Example 19 (continued from Example 1). By Example 15, we get $D_{\widehat{\mathrm{C}}_{1}}^{0.6}$ and $D_{\widehat{\mathrm{C}}_{2}}^{0.6}$. Then,

$$
S \underline{R}_{\sum_{i=1}^{O} \widehat{\mathbf{C}}_{i}}^{2.3}(A)=\bigvee_{i=1}^{2}\left(\left(D_{\widehat{\mathbf{C}}_{i}}^{0.6}\right)^{0.3} \triangleright A \odot 1_{5 \times 1}\right)
$$




$$
\begin{aligned}
& =\left(\left(\begin{array}{lllll}
1 & 0 & 1 & 0 & 1 \\
0 & 1 & 0 & 1 & 1 \\
1 & 0 & 1 & 0 & 0 \\
0 & 1 & 0 & 1 & 0 \\
1 & 1 & 0 & 0 & 1
\end{array}\right) \triangleright\left(\begin{array}{c}
0.8 \\
0.4 \\
0.6 \\
0.2 \\
0.5
\end{array}\right) \odot\left(\begin{array}{l}
1 \\
1 \\
1 \\
1 \\
1
\end{array}\right) \vee\left(\left(\begin{array}{lllll}
1 & 1 & 1 & 0 & 0 \\
1 & 1 & 1 & 0 & 0 \\
1 & 1 & 1 & 0 & 0 \\
0 & 0 & 0 & 1 & 1 \\
0 & 0 & 0 & 1 & 1
\end{array}\right) \triangleright\left(\begin{array}{l}
0.8 \\
0.4 \\
0.6 \\
0.2 \\
0.5
\end{array}\right)\left(\begin{array}{l}
1 \\
1 \\
1 \\
1 \\
1
\end{array}\right)\right)\right. \\
& =\left(\left(\begin{array}{ccccc}
0.8 & 1 & 0.6 & 1 & 0.5 \\
1 & 0.4 & 1 & 0.2 & 0.5 \\
0.8 & 1 & 0.6 & 1 & 1 \\
1 & 0.4 & 1 & 0.2 & 1 \\
0.8 & 0.4 & 1 & 1 & 0.5
\end{array}\right) \odot\left(\begin{array}{l}
1 \\
1 \\
1 \\
1 \\
1
\end{array}\right) \bigvee\left(\left(\begin{array}{ccccc}
0.8 & 0.4 & 0.6 & 1 & 1 \\
0.8 & 0.4 & 0.6 & 1 & 1 \\
0.8 & 0.4 & 0.6 & 1 & 1 \\
1 & 1 & 1 & 0.2 & 0.5 \\
1 & 1 & 1 & 0.2 & 0.5
\end{array}\right) \odot\left(\begin{array}{l}
1 \\
1 \\
1 \\
1 \\
1
\end{array}\right)\right)\right. \\
& =\left(\begin{array}{c}
0.5 \\
0.2 \\
0.6 \\
0.2 \\
0.4
\end{array}\right) \bigvee\left(\begin{array}{c}
0.4 \\
0.4 \\
0.4 \\
0.2 \\
0.2
\end{array}\right)=\left(\begin{array}{c}
0.5 \\
0.4 \\
0.6 \\
0.2 \\
0.4
\end{array}\right), \\
& S \bar{R}_{\sum_{i=1}^{2} \widehat{\mathbf{C}}_{i}}^{2,0.3}(A)=\bigwedge_{i=1}^{2}\left(\left(D_{\widehat{\mathbf{C}}_{i}}^{0.6}\right)^{0.3} \triangleleft A \circ 0_{5 \times 1}\right) \\
& =\left(\left(\begin{array}{lllll}
1 & 0 & 1 & 0 & 1 \\
0 & 1 & 0 & 1 & 1 \\
1 & 0 & 1 & 0 & 0 \\
0 & 1 & 0 & 1 & 0 \\
1 & 1 & 0 & 0 & 1
\end{array}\right) \triangleleft\left(\begin{array}{l}
0.8 \\
0.4 \\
0.6 \\
0.5
\end{array}\right) \circ\left(\begin{array}{l}
0 \\
0 \\
0 \\
0 \\
0
\end{array}\right) \wedge\left(\left(\begin{array}{lllll}
1 & 1 & 1 & 0 & 0 \\
1 & 1 & 1 & 0 & 0 \\
1 & 1 & 1 & 0 & 0 \\
0 & 0 & 0 & 1 & 1 \\
0 & 0 & 0 & 1 & 1
\end{array}\right) \triangleleft\left(\begin{array}{l}
0.8 \\
0.4 \\
0.6 \\
0.2 \\
0.5
\end{array}\right) \circ\left(\begin{array}{l}
0 \\
0 \\
0 \\
0 \\
0
\end{array}\right)\right)\right. \\
& =\left(\begin{array}{l}
0.8 \\
0.5 \\
0.8 \\
0.4 \\
0.5
\end{array}\right) .
\end{aligned}
$$

Inspired by Definition 8 and Proposition 9, we present the matrix representations of the $\alpha$-PMNFLAO $\underline{R}_{\sum_{i=1}^{P, \alpha} \widehat{\mathrm{C}}_{i}}(A)$ and the $\alpha$-PMNFUAO $\bar{R}_{\sum_{i=1}^{n, \alpha} \widehat{\mathrm{C}}_{i}}(A)$ as follows.

Theorem 6. Let $\widetilde{\Gamma}=\left\{\widehat{\mathbf{C}}_{1}, \ldots, \widehat{\mathbf{C}}_{n}\right\}$ be an $n$-fuzzy $\beta$-coverings of $U=\left\{x_{1}, \ldots, x_{m}\right\}$ and $\alpha \in[0,1]$. Then, for any $A \in F(U)$,

$$
\begin{aligned}
& \underline{R}_{\sum_{i=1}^{P} \widehat{\mathbf{C}}_{i}}^{P, \alpha}(A)=\bigwedge_{i=1}^{n}\left(\left(D_{\widehat{\mathbf{C}}_{i}}^{\beta}\right)^{\alpha} \odot 1_{m \times 1}\right), \\
& \bar{R}_{\sum_{i=1}^{n} \widehat{\mathbf{C}}_{i}}(A)=\bigvee_{i=1}^{n}\left(\left(D_{\widehat{\mathbf{C}}_{i}}^{\beta}\right)^{\alpha} \circ 0_{m \times 1}\right),
\end{aligned}
$$

where $\triangleright$ and $\triangleleft$ are first than $\odot$ and $\circ$, respectively. 
Proof. By Definition 8 and Proposition 9, it is immediate.

Example 20 (continued from Example 1). By Example 15, we get $D_{\widehat{\mathrm{C}}_{1}}^{0.6}$ and $D_{\widehat{\mathrm{C}}_{2}}^{0.6}$. Then,

$$
\begin{aligned}
& S \underline{R}_{\sum_{i=1}^{P, 0.3} \widehat{\mathbf{C}}_{i}}^{2}(A)=\bigwedge_{i=1}^{2}\left(\left(D{\widehat{\widehat{C}_{i}}}^{0.6}\right)^{0.3} \triangleright A \odot 1_{5 \times 1}\right) \\
& =\left(\left(\begin{array}{lllll}
1 & 0 & 1 & 0 & 1 \\
0 & 1 & 0 & 1 & 1 \\
1 & 0 & 1 & 0 & 0 \\
0 & 1 & 0 & 1 & 0 \\
1 & 1 & 0 & 0 & 1
\end{array}\right) \triangleright\left(\begin{array}{c}
0.8 \\
0.4 \\
0.6 \\
0.2 \\
0.5
\end{array}\right) \odot\left(\begin{array}{l}
1 \\
1 \\
1 \\
1 \\
1
\end{array}\right) \wedge\left(\left(\begin{array}{lllll}
1 & 1 & 1 & 0 & 0 \\
1 & 1 & 1 & 0 & 0 \\
1 & 1 & 1 & 0 & 0 \\
0 & 0 & 0 & 1 & 1 \\
0 & 0 & 0 & 1 & 1
\end{array}\right) \triangleright\left(\begin{array}{l}
0.8 \\
0.4 \\
0.6 \\
0.2 \\
0.5
\end{array}\right) \odot\left(\begin{array}{l}
1 \\
1 \\
1 \\
1 \\
1
\end{array}\right)\right)\right. \\
& =\left(\left(\begin{array}{ccccc}
0.8 & 1 & 0.6 & 1 & 0.5 \\
1 & 0.4 & 1 & 0.2 & 0.5 \\
0.8 & 1 & 0.6 & 1 & 1 \\
1 & 0.4 & 1 & 0.2 & 1 \\
0.8 & 0.4 & 1 & 1 & 0.5
\end{array}\right) \odot\left(\begin{array}{l}
1 \\
1 \\
1 \\
1 \\
1
\end{array}\right) \wedge\left(\left(\begin{array}{ccccc}
0.8 & 0.4 & 0.6 & 1 & 1 \\
0.8 & 0.4 & 0.6 & 1 & 1 \\
0.8 & 0.4 & 0.6 & 1 & 1 \\
1 & 1 & 1 & 0.2 & 0.5 \\
1 & 1 & 1 & 0.2 & 0.5
\end{array}\right) \odot\left(\begin{array}{l}
1 \\
1 \\
1 \\
1 \\
1
\end{array}\right)\right)\right. \\
& =\left(\begin{array}{l}
0.5 \\
0.2 \\
0.6 \\
0.2 \\
0.4
\end{array}\right) \wedge\left(\begin{array}{l}
0.4 \\
0.4 \\
0.4 \\
0.2 \\
0.2
\end{array}\right)=\left(\begin{array}{l}
0.4 \\
0.2 \\
0.4 \\
0.2 \\
0.2
\end{array}\right) \\
& S \bar{R}_{\sum_{i=1}^{2}, 0.3}^{2} \widehat{\mathbf{C}}_{i}(A)=\bigvee_{i=1}^{2}\left(\left(D_{\widehat{\mathrm{C}}_{i}}^{0.6}\right)^{0.3} \triangleleft A \circ 0_{5 \times 1}\right) \\
& =\left(\left(\begin{array}{lllll}
1 & 0 & 1 & 0 & 1 \\
0 & 1 & 0 & 1 & 1 \\
1 & 0 & 1 & 0 & 0 \\
0 & 1 & 0 & 1 & 0 \\
1 & 1 & 0 & 0 & 1
\end{array}\right) \triangleleft\left(\begin{array}{l}
0.8 \\
0.4 \\
0.6 \\
0.2 \\
0.5
\end{array}\right) \circ\left(\begin{array}{l}
0 \\
0 \\
0 \\
0 \\
0
\end{array}\right) \vee\left(\left(\begin{array}{lllll}
1 & 1 & 1 & 0 & 0 \\
1 & 1 & 1 & 0 & 0 \\
1 & 1 & 1 & 0 & 0 \\
0 & 0 & 0 & 1 & 1 \\
0 & 0 & 0 & 1 & 1
\end{array}\right) \triangleleft\left(\begin{array}{l}
0.8 \\
0.4 \\
0.6 \\
0.2 \\
0.5
\end{array}\right) \circ\left(\begin{array}{l}
0 \\
0 \\
0 \\
0 \\
0
\end{array}\right)\right)\right. \\
& =\left(\begin{array}{l}
0.8 \\
0.8 \\
0.8 \\
0.5 \\
0.8
\end{array}\right) \text {. }
\end{aligned}
$$

To evaluate the efficiency of our matrix method for computing covering-based multigranulation fuzzy rough approximation operators, we choose three datasets from UCI machine learning repository. Their computational time is shown in Table 9. They are carried out on a personal computer with 64-bit Windows 7, Intel ${ }^{\circledR}$ Core $^{\text {тм }}$ i5-4460 CPU @3.20 GHz, and 8GB memory. The programming language is MATLAB r2016a. Since the method of attribute reduction is always presented by rough approximation operators, our matrix method for computing covering-based multigranulation fuzzy rough approximation operators can be used to solve this problem in the future.

\section{An Application in MCGDM}

In this section, these CMFRS models are applied to manage the problem of MCGDM that is stated in [31]. Zhan et al. [31] presented an algorithm for the problem of MCGDM based on the covering-based optimistic multigranulation fuzzy rough set model. We show it in Algorithm 1, where $\widetilde{\Gamma}$ is an $n$-fuzzy $\beta$-coverings of $U$ and $\mu \in F(U)$. 
TABLE 9: Comparison of different approximation operators for some datasets in UCI machine learning repository.

\begin{tabular}{|c|c|c|c|c|c|c|}
\hline Datasets & Samples & Attributes & Operators & $\begin{array}{c}\text { Computational time of } 30 \% \\
\text { U (s) }\end{array}$ & $\begin{array}{c}\text { Computational time of } 60 \% \\
\text { U (s) }\end{array}$ & $\begin{array}{c}\text { Computational time of } 100 \% \\
\mathrm{U}(\mathrm{s})\end{array}$ \\
\hline \multirow{4}{*}{ Iris } & \multirow{4}{*}{150} & \multirow{4}{*}{4} & $S \underline{R}_{\Gamma^{n}}^{O} \widehat{\mathrm{C}}$ & 0.0361 & 0.3375 & 0.3631 \\
\hline & & & $S \bar{R}_{\sum^{n} \widehat{C}_{i=1}}^{\hat{C}_{i}}$ & 0.0355 & 0.3095 & 0.3195 \\
\hline & & & $\underline{R}_{\sum_{i=1}^{n}}^{\sum_{i=1}^{O, \alpha} \widehat{C}_{i}}$ & 0.0454 & 0.3000 & 0.3478 \\
\hline & & & $\bar{R}_{\sum_{i=1}^{n, \alpha} \widehat{C}_{i}}$ & 0.0375 & 0.4373 & 0.4138 \\
\hline \multirow{4}{*}{$\begin{array}{l}\text { Forest } \\
\text { fires }\end{array}$} & \multirow{4}{*}{517} & \multirow{4}{*}{7} & $S \underline{R}_{\Gamma^{n}}^{O}$ & 0.9938 & 2.7521 & 9.3000 \\
\hline & & & $S \bar{R}_{\sum_{i=1}^{n} \widehat{\mathbf{C}}_{i}}^{O}$ & 0.6449 & 2.7275 & 10.5721 \\
\hline & & & $\underline{R}_{\sum_{i=1}^{n}}^{\mathcal{L}_{i=1} \widehat{C}_{i}}$ & 0.9039 & 2.5587 & 10.6041 \\
\hline & & & $\bar{R}_{\sum_{i=1}^{O, \alpha} \widehat{C}_{i}}$ & 0.7335 & 2.4848 & 10.6961 \\
\hline \multirow{4}{*}{ Blood } & \multirow{4}{*}{748} & \multirow{4}{*}{4} & $S \underline{R}_{\Gamma^{n}}^{O} \widehat{\mathrm{C}}$ & 0.7248 & 4.0976 & 22.2954 \\
\hline & & & $S \bar{R}_{\sum^{n} \widehat{\mathbf{C}}_{i}}^{O L_{i=1} \hat{C}_{i}}$ & 0.6664 & 4.0277 & 22.5706 \\
\hline & & & $\underline{R}_{\sum_{i=1}^{n}}^{L_{i=1} \widehat{C}_{i}}$ & 0.7492 & 4.1257 & 21.9894 \\
\hline & & & $\bar{R}_{\sum_{i=1}^{n}{ }^{n}, \alpha}^{\mathrm{C}_{i}}$ & 0.7285 & 3.8302 & 22.5545 \\
\hline
\end{tabular}

Input: a multigranulation fuzzy decision information system $(U, \widetilde{\Gamma}, \beta, \mu)$.

Output: the rank of all alternatives.

Step 1: by Definition 2, calculating all $\widetilde{N}_{\widehat{\mathrm{C}}_{i}(x)}^{\beta}$ for any $x \in U$ and $i=1,2, \ldots, n$.

Step 2: by Definition 3, computing $\underline{R}_{\sum_{i=1}^{n}}^{O} \widehat{\mathrm{c}}_{i}(\mu)$ and $\bar{R}_{\sum_{i=1}^{O} \widehat{\mathrm{c}}_{i}}(\mu)$, respectively.

Step 3: getting the right weight value of $\zeta$, where $\zeta \in[0,1]$.

Step 4: calculating $\sum_{i=1}^{n} \widetilde{R}_{i}^{O}(\mu)=\zeta \underline{R}_{\sum_{i=1}^{n} \widehat{\mathrm{c}}_{i}}^{O}(\mu)+(1-\zeta) \bar{R}_{\sum_{i=1}^{O} \widehat{\mathrm{c}}_{i}}(\mu)$.

Step 5: obtaining the ordering by the decision principle.

Algorithm 1: An algorithm for the problem of MCGDM in [31].

Inspired by Algorithm 1, we use the OMNFLAO $S \underline{R}_{\sum_{i=1}^{n} \widehat{\mathrm{C}}_{i}}(\mu)$ and OMNFUAO $S \bar{R}_{\sum_{i=1}^{n} \widehat{\mathrm{C}}_{i}}(\mu)$ that are presented in this paper to replace $\underline{R}_{\sum_{i=1}^{n} \widehat{\mathrm{C}}_{i}}(\mu)$ and $\bar{R}_{\sum_{i=1}^{n} \widehat{\mathrm{C}}_{i}}(\mu)$, respectively. Hence, Algorithm 2 is proposed as follows:

On the other hand, Algorithm 3 is proposed based on Definition 6 to solve the problem of MCGDM.

To show the validity of our method, we compare Algorithms 1-3 in the following example.

Example 21. Let $U=\left\{x_{1}, x_{2}, \ldots, x_{5}\right\}$ be a set of patients, $\beta=$ 0.6 be the critical value, and $V=\left\{y_{1}, y_{2}, y_{3}, y_{4}\right\}$ be a set of five main symptoms for the disease $A$. Suppose that the doctor $X$ invites 2 experts $R_{i}(i=1,2)$ to evaluate every patient $x_{k}$ $(k=1,2, \ldots, 5)$. Then, the expert $R_{i}$ believes that the patient $x_{k}$ has a value of the symptom $y_{j}(j=1, \ldots, 4)$, denoted as $C_{i j}\left(x_{k}\right)$. For the patient $x_{k}$, there exists at least one symptom $y_{j}$ such that the symptom value $C_{i j}\left(x_{k}\right) \geq \beta$. Then, $\widetilde{\Gamma}=\left\{\widehat{\mathbf{C}}_{1}, \widehat{\mathbf{C}}_{2}\right\}$ is a family of fuzzy $\beta$-coverings of $U$, where $\widehat{\mathbf{C}}_{i}=\left\{C_{i 1}\right.$, $\left.C_{i 2}, C_{i 3}, C_{i 4}\right\}(i=1,2)$. They are shown in Tables 1 and 9 , respectively. Suppose that the doctor $X$ gives the possible value $\mu$ of the disease $A$ of every patient as $\mu=\left(0.8 / x_{1}\right)+(0.4 /$ $\left.x_{2}\right)+\left(0.6 / x_{3}\right)+\left(0.2 / x_{4}\right)+\left(0.5 / x_{5}\right)$. The doctor $X$ wants to decide whether or not the patients $x_{k} \in U$ have the disease $A$.

Firstly, we use Algorithm 1 to solve this problem as follows:

Step 1: all $\widetilde{N}_{\widehat{C}_{i}\left(x_{k}\right)}^{0.6}$ are shown in Tables 3 and 4, for any $i=1,2$ and $k=1,2, \ldots, 5$

Step 2: $\underline{R}_{\sum_{i=1}^{O} \widehat{\mathrm{c}}_{i}}(\mu)=\left(0.5 / x_{1}\right)+\left(0.4 / x_{2}\right)+\left(0.6 / x_{3}\right)+$ $\left(0.4 / x_{4}\right)+\left(0.5 / x_{5}\right)$, and $\bar{R}_{\sum_{i=1}^{O} \widehat{C}_{i}}(\mu)=\left(0.7 / x_{1}\right)+(0.5 /$ $\left.x_{2}\right)+\left(0.6 / x_{3}\right)+\left(0.4 / x_{4}\right)+\left(0.5 / x_{5}\right)$

Step 3: $\zeta=0.3$

Step 4: $\sum_{i=1}^{2} \widetilde{R}_{i}^{O}(\mu)=\left(0.64 / x_{1}\right)+\left(0.47 / x_{2}\right)+\left(0.6 / x_{3}\right)+$ $\left(0.4 / x_{4}\right)+\left(0.5 / x_{5}\right)$ 
Input: a multigranulation fuzzy decision information system $(U, \widetilde{\Gamma}, \beta, \mu)$.

Output: the rank of all alternatives.

Step 1: by Definition 4, calculating all $D_{\widehat{\mathrm{C}}_{i}}^{\beta}(x, y)$ for any $x \in U$ and $i=1,2, \ldots, n$.

Step 2: by Definition 3, computing

$S \underline{R}_{\sum_{i=1}^{n}}^{O} \widehat{\mathbf{c}}_{i}(\mu)(x)=\vee_{i=1}^{n} \wedge_{y \in U}\left\{\left(1-D_{\widehat{\mathrm{C}}_{i}}^{\beta}(x, y)\right) \vee \mu(y)\right\}$,

$S \bar{R}_{\sum_{i=1}^{n} \widehat{\mathbf{c}}_{i}}^{O}(\mu)(x)=\wedge_{i=1}^{n} \vee_{y \in U}\left\{D_{\widehat{\mathrm{C}}_{i}}^{\beta}(x, y) \wedge \mu(y)\right\}$.

Step 3: getting the right weight value of $\zeta$, where $\zeta \in[0,1]$.

Step 4: calculating $\sum_{i=1}^{n} S \widetilde{R}_{i}^{O}(\mu)=\zeta S \underline{R}_{\sum_{i=1}^{n}}^{O} \widehat{\mathrm{c}}_{i}(\mu)+(1-\zeta) S R_{\sum_{i=1}^{n} \widehat{\mathrm{c}}_{i}}^{O}(\mu)$.

Step 5: obtaining the ordering by the decision principle.

Algorithm 2: An algorithm for the problem of MCGDM.

Input: a multigranulation fuzzy decision information system $(U, \widetilde{\Gamma}, \beta, \mu)$.

Output: the rank of all alternatives.

Step 1: by Definition 4, calculating all $D_{\widehat{\mathrm{C}}_{i}}^{\beta}(x, y)$ for any $x \in U$ and $i=1,2, \ldots, n$.

Step 2: getting the right weight value of $\zeta$, where $\zeta \in[0,1]$.

Step 3: by Definition 3, computing

$\underline{R}^{O, \zeta} \sum_{i=1}^{n} \widehat{\mathrm{C}}_{i}(\mu)(x)=\vee_{i=1}^{n} \wedge_{y \in U}\left\{\mu(y): D_{\widehat{\mathrm{C}}_{i}}^{\beta}(x, y) \geq \zeta\right\}$,

$\bar{R}_{\sum_{i=1}^{n} \widehat{C}_{i}}(A)(x)=\wedge_{i=1}^{n} \vee_{y \in U}\left\{\mu(y): D_{\widehat{C}_{i}}^{\beta}(x, y) \geq \zeta\right\}$.

Step 4: calculating $\sum_{i=1}^{n} \widetilde{R}_{i}^{O}(\mu)=\zeta S \underline{R}_{\sum_{i=1}^{n} \widehat{\mathrm{c}}_{i}}(\mu)+(1-\zeta) \bar{R}_{\sum_{i=1}^{O} \widehat{\mathrm{c}}_{i}}(\mu)$.

Step 5: obtaining the ordering by the decision principle.

Algorithm 3: An algorithm for the problem of MCGDM.

Table 10: The results utilizing the different methods of Example 21.

\begin{tabular}{lcc}
\hline Methods & The final ranking & The patients are more likely to be sicken for the disease $A$ \\
\hline Algorithm 1 (Zhan et al. [31]) & $x_{4} \prec x_{2} \prec x_{5} \prec x_{3} \prec x_{1}$ & $x_{1}$ \\
Algorithm 2 & $x_{4} \prec x_{2} \prec x_{5} \prec x_{3} \prec x_{1}$ & $x_{1}$ \\
Algorithm 3 & $x_{4} \prec x_{2} \approx x_{5} \prec x_{1} \prec x_{3}$ & $x_{3}$ \\
\hline
\end{tabular}

Step 5: we get $x_{4} \prec x_{2} \prec x_{5} \prec x_{3} \prec x_{1}$. That is to say, $x_{1}$ is more likely to be sicken for the disease $A$

Then, for Algorithm 2:

Step 1: $D_{\widehat{\mathrm{C}}_{1}}^{0.6}$ and $D_{\widehat{\mathrm{C}}_{2}}^{0.6}$ are shown in Tables 7 and 8, respectively

Step 2: $S \underline{R}_{\sum_{i=1}^{O}}^{O} \widehat{\mathrm{c}}_{i}(\mu)=\left(0.6667 / x_{1}\right)+\left(0.4 / x_{2}\right)+\left(0.6 / x_{3}\right)$

$+\left(0.2 / x_{4}\right)+\left(0.5 / x_{5}\right)$, and $S \bar{R}_{\sum_{i=1}^{2} \widehat{C}_{i}}^{O}(\mu)=\left(0.8 / x_{1}\right)+$

$\left(0.4 / x_{2}\right)+\left(0.6 / x_{3}\right)+\left(0.333 / x_{4}\right)+\left(0.5 / x_{5}\right)$

Step 3: $\zeta=0.3$

Step 4: $\sum_{i=1}^{2} S \widetilde{R}_{i}^{O}(\mu)=\left(0.76 / x_{1}\right)+\left(0.4 / x_{2}\right)+\left(0.6 / x_{3}\right)+$ $\left(0.2933 / x_{4}\right)+\left(0.5 / x_{5}\right)$

Step 5: we get $x_{4} \prec x_{2} \prec x_{5} \prec x_{3} \prec x_{1}$. That is to say, $x_{1}$ is more likely to be sicken for the disease $A$
For Algorithm 3:

Step 1: $D_{\widehat{\mathrm{C}}_{1}}^{0.6}$ and $D_{\widehat{\mathrm{C}}_{2}}^{0.6}$ are shown in Tables 7 and 8, respectively

Step 2: $\zeta=0.3$

Step 3: $\underline{R}_{\sum_{i=1}^{O}, 0.3}^{2} \widehat{\mathrm{c}}_{i}(\mu)=\left(0.5 / x_{1}\right)+\left(0.4 / x_{2}\right)+\left(0.6 / x_{3}\right)+$ $\left(0.2 / x_{4}\right)+\left(0.4 / x_{5}\right), \quad$ and $\quad \bar{R}_{\sum_{i=1}^{2} \widehat{C}_{i}}^{2,3}(\mu)=\left(0.8 / x_{1}\right)+$ $\left(0.5 / x_{2}\right)+\left(0.8 / x_{3}\right)+\left(0.4 / x_{4}\right)+\left(0.5 / x_{5}\right)$

Step 4: $\sum_{i=1}^{2} \widetilde{R}_{i}^{O, 0.3}(\mu)=\left(0.71 / x_{1}\right)+\left(0.47 / x_{2}\right)+$

$\left.x_{3}\right)+\left(0.34 / x_{4}\right)+\left(0.47 / x_{5}\right)$

Step 5: we get $x_{4} \prec x_{2} \approx x_{5} \prec x_{1} \prec x_{3}$. That is to say, $x_{3}$ is more likely to be sicken for the disease $A$

Finally, all results about are shown in Table 10 and Figure 1. From Table 10 and Figure 1, we find that the results 


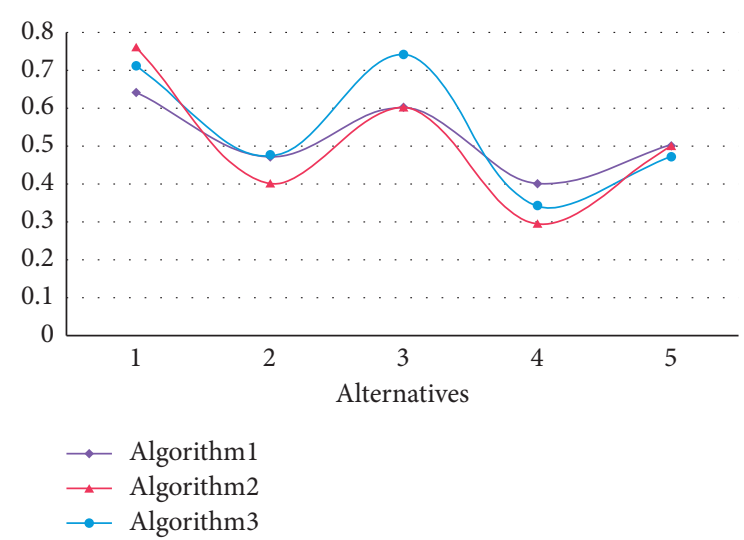

FIgURE 1: Comparisons of the ranking results in Example 21 based on Algorithms 1-3.

of Algorithms 1 and 2 are same. That is to say, the patient $x_{1}$ is more likely to be sicken for the disease $A$. Hence, our methods are effective. On the other hand, $x_{3}$ is more likely to be sicken for the disease $A$ by Algorithm 3. It is different with other methods. So, it will be a new choice for the decision maker.

\section{Conclusions}

This paper constructs some types of CMFRS models through fuzzy neighborhood measures. Corresponding fast calculation methods based on matrices are also proposed. Moreover, as an application, they are used to solve the problem of MCGDM. The main contributions of this paper are concluded as follows:

(1) It is interesting to give the notion of multigranulation fuzzy neighborhood measure based on the concept of $\beta$-neighborhood. It can be seen as the complement of the existing work and also the dual of multigranulation fuzzy measure which is presented in [31]. These new types of CMFRS models can be seen as the combination of covering-based rough sets, multigranulation rough sets and fuzzy sets. These models are the complement of the existing rough set models. They can help us to deal with multigranulation fuzzy information well.

(2) It is complicated and time-consuming to use set approaches to compute these models in a large cardinal fuzzy $\beta$-covering approximation space. The matrix methods can satisfy the needs of knowledge discovery. By using matrix approaches in CMFRS models, calculations can be easily implemented by computers. It can well solve the time-consuming problem when a large-scale covering is given.

(3) According to Algorithm 3, we find that the patient $x_{3}$ is another patient that is more likely to be sicken for the disease $A$ in Example 21, which is different from Algorithms 1 and 2. That is to say, it is a new choice for decision maker. Therefore, our method is a complement of the existing methods.
In future research, the reduction in covering-based multigranulation fuzzy information systems should be investigated, as well as the matrix calculation approach. The generalizations of fuzzy sets (such as soft multisets [32], picture fuzzy sets [33], and neutrosophic sets [34]) will be combined with the content of this paper. Moreover, it can be used in the problem of MCGDM.

\section{Data Availability}

The data are available at UCI machine learning repository (http://archive.ics.uci.edu/ml/index.php).

\section{Conflicts of Interest}

The authors declare that they have no conflicts of interest regarding the publication of this study.

\section{Acknowledgments}

This work was supported by the Scientific Research Program Funded by Shaanxi Provincial Education Department (Program no. 21JK0749) and the Scientific Research Program Funded by Shaanxi Provincial Science and Technology Department (Program no. 2021JQ-894).

\section{References}

[1] W. Zhu and F.-Y. Wang, "Reduction and axiomization of covering generalized rough sets," Information Sciences, vol. 152, pp. 217-230, 2003.

[2] A. Tan, J. Li, and G. Lin, "Connections between coveringbased rough sets and concept lattices," International Journal of Approximate Reasoning, vol. 56, pp. 43-58, 2015.

[3] Z. a. Pawlak, "Rough sets," International Journal of Computer \& Information Sciences, vol. 11, no. 5, pp. 341-356, 1982.

[4] X. Zhang, J. Dai, and Y. Yu, "On the union and intersection operations of rough sets based on various approximation spaces," Information Sciences, vol. 292, pp. 214-229, 2015.

[5] L. Ma, "The investigation of covering rough sets by Boolean matrices," International Journal of Approximate Reasoning, vol. 100, pp. 69-84, 2018.

[6] J. Wang and X. Zhang, "Matrix approaches for some issues about minimal and maximal descriptions in covering-based rough sets," International Journal of Approximate Reasoning, vol. 104, pp. 126-143, 2019.

[7] Y. Jing, T. Li, H. Fujita, Z. Yu, and B. Wang, "An incremental attribute reduction approach based on knowledge granularity with a multi-granulation view," Information Sciences, vol. 411, pp. 23-38, 2017.

[8] C. Wang, Y. Shi, X. Fan, and M. Shao, "Attribute reduction based on $k$-nearest neighborhood rough sets $\kappa$-nearest neighborhood rough sets," International Journal of Approximate Reasoning, vol. 106, pp. 18-31, 2019.

[9] Y. Du, Q. Hu, P. Zhu, and P. Ma, "Rule learning for classification based on neighborhood covering reduction," Information Sciences, vol. 181, no. 24, pp. 5457-5467, 2011.

[10] J. Wang and W. Zhu, "Applications of bipartite graphs and their adjacency matrices to covering-based rough sets," Fundamenta Informaticae, vol. 156, no. 2, pp. 237-254, 2017.

[11] L. A. Zadeh, "Fuzzy sets," Information and Control, vol. 8, no. 3, pp. 338-353, 1965. 
[12] Y. Yao, "A comparative study of fuzzy sets and rough sets," Information Sciences, vol. 109, no. 1-4, pp. 227-242, 1998.

[13] D. Dubois and H. Prade, "Rough fuzzy sets and fuzzy rough sets," International Journal of General Systems, vol. 17, no. 2-3, pp. 191-209, 1990.

[14] K. T. Atanassov, "Intuitionistic fuzzy sets," Fuzzy Sets and Systems, vol. 20, no. 1, pp. 87-96, 1986.

[15] D. Molodtsov, "Soft set theory-first results," Computers \& Mathematics with Applications, vol. 37, no. 4-5, pp. 19-31, 1999.

[16] S. Preveena and M. Kamaraj, "On bipolar valued fuzzy sets and their operations," Asian Journal of Science and Technology, vol. 9, pp. 8557-8564, 2018.

[17] T. Mahmood, "A novel approach towards bipolar soft sets and their applications," Journal of Mathematics, vol. 2020, Article ID 4690808, 11 pages, 2020.

[18] J. Wang, Y. Wang, and L. Liu, "Hesitant bipolar-valued fuzzy soft sets and their application in decision making," Journal of Mathematics, vol. 2020, Article ID 6496030, 12 pages, 2020.

[19] H. Barrenechea, E. Barrenechea, M. Pagola et al., "A historical account of types of fuzzy sets and their relationships," IEEE Transactions on Fuzzy Systems, vol. 24, pp. 179-194, 2016.

[20] H. Wang, F. Smarandache, Y. Zhang et al., "Single valued neutrosophic sets," Multispace and Multistructure, vol. 4, pp. 410-413, 2010.

[21] T. Feng, S.-P. Zhang, and J.-S. Mi, "The reduction and fusion of fuzzy covering systems based on the evidence theory," International Journal of Approximate Reasoning, vol. 53, no. 1, pp. 87-103, 2012.

[22] T.-J. Li, Y. Leung, and W.-X. Zhang, "Generalized fuzzy rough approximation operators based on fuzzy coverings," International Journal of Approximate Reasoning, vol. 48, no. 3, pp. 836-856, 2008.

[23] B. Šešelja, "L-fuzzy covering relation," Fuzzy Sets and Systems, vol. 158, pp. 2456-2465, 2007.

[24] L. Ma, "Two fuzzy covering rough set models and their generalizations over fuzzy lattices," Fuzzy Sets and Systems, vol. 294, pp. 1-17, 2016.

[25] X. Zhang and J. Wang, "Fuzzy $\beta$-covering approximation spaces $\beta$-covering approximation spaces," International Journal of Approximate Reasoning, vol. 126, pp. 27-47, 2020.

[26] L. D'eer, C. Cornelis, and L. Godo, "Fuzzy neighborhood operators based on fuzzy coverings," Fuzzy Sets and Systems, vol. 312 , pp. 17-35, 2017.

[27] L. D'eer and C. Cornelis, “A comprehensive study of fuzzy covering-based rough set models: definitions, properties and interrelationships," Fuzzy Sets and Systems, vol. 336, pp. 1-26, 2018.

[28] B. Yang and B. Q. Hu, "A fuzzy covering-based rough set model and its generalization over fuzzy lattice," Information Sciences, vol. 367-368, pp. 463-486, 2016.

[29] B. Yang and B. Q. Hu, "Fuzzy neighborhood operators and derived fuzzy coverings," Fuzzy Sets and Systems, vol. 370, pp. 1-33, 2019.

[30] X. Zhang, J. Wang, J. Zhan, and J. Dai, "Fuzzy measures and choquet integrals based on fuzzy covering rough sets," IEEE Transactions on Fuzzy Systems, 2021.

[31] J. Zhan, X. Zhang, and Y. Yao, "Covering based multigranulation fuzzy rough sets and corresponding applications," Artificial Intelligence Review, vol. 53, no. 2, pp. 1093-1126, 2020.

[32] M. Riaz, N. Agman, N. Çagman, N. Wali, and A. Mushtaq, "Certain properties of soft multi-set topology with applications in multi-criteria decision making," Decision Making:
Applications in Management and Engineering, vol. 3, no. 2, pp. 70-96, 2020.

[33] S. R. Dash and S. Das, "Career selection of students using hybridized distance measure based on picture fuzzy set and rough set theory," Decision Making Applications in Management and Engineering, vol. 4, pp. 104-126, 2021.

[34] J. Wang and X. Zhang, "Multigranulation single valued neutrosophic covering-based rough sets and their applications to multi-criteria group decision making," Iranian Journal of Fuzzy Systems, vol. 17, pp. 109-126, 2020. 